\title{
The effect of plant-fungi interaction generalism on plant community productivity
}

\author{
By \\ Jacob Thomson-Laing
}

A thesis submitted to Victoria University of Wellington in partial fulfilment of the requirements for the degree of Master of Science in Ecology and Biodiversity

TE WHARE WĀNANGA O TE ÜPOKO O TE IKA A MĀUI

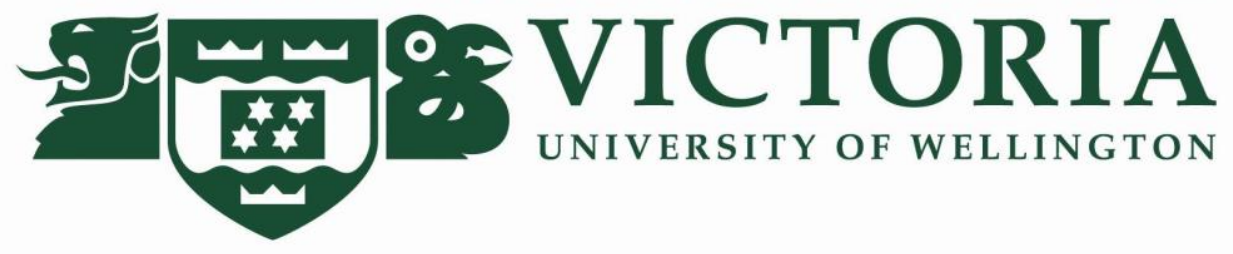

Victoria University of Wellington 
Dedicated to my twin 


\begin{abstract}
Plant-plant productivity relationships within ecosystem and community ecology are contentiously debated in the literature due to the numerous factors involved making conclusions hard to draw and disentangle. There are several widely established and supported plant-plant productivity relationships. Increasing species richness can allow for greater niche complementarity, which in turn increases overall above and below ground productivity. Plants with different functional traits can differentially affect a plant community depending on the arrival time of the plant. These priority effects allow certain plants to outcompete others and persist in a community across different temporal scales. Plant species differ in their ability to interact with certain species of symbiotic partners in the soil (Arbuscular mycorrhizal fungi, AMF). This interaction generalism of a plant species indicates the ability of a plant to host many or few AMF species (generalist or specialist, respectively). However, there remains a limited understanding of plant-fungi relationships especially with respect to community productivity and the temporal effects of adding contrasting types of interaction generalism into an established community.
\end{abstract}

The aim of this study was to determine the effects of the addition of an interaction specialist or generalist plant species into an established plant community on the overall community productivity. Three communities that differed in plant species richness were grown for 38 days at which point either a generalist or specialist was added. Community treatments were carried out in field soil, sterile soil and sterile soil reinoculated with viable field soil, separating the effects of plant niche-partitioning for plant-fungi interaction partners from the effects of niche-partitioning for other resources (e.g. soil nutrients). Community productivity was tested using different productivity measures; 1) carbon flux as the Net Ecosystem Exchange (NEE) of the community, 2) total above and below ground plant biomass, 3) neutral lipid fatty acid (NLFA) AMF biomarker, 16:1w5, extracted from total soil and total root mass to assess AMF biomass.

It was difficult to disentangle the effects of species richness and interaction generalism on carbon flux in communities, with soil type clearly impacting these relationships. In all soil types, an increase in community plant richness had the greatest effect on carbon draw down and biomass productivity with respect to both plant and AMF biomass. In non-sterilised soil, interaction generalism, specifically the addition of a specialist alongside increased species richness corresponded to increased carbon drawdown. In the context of previous research, this study further highlighted the complexity of factors driving plant-plant-fungi relationships, but clearly identifies the positive role that species richness is having. Although the role of plantfungi relationships in overall community productive remains unclear, this study provides a platform for future research to be undertaken. 


\section{Acknowledgements}

This thesis has been a group effort and one where many others need to be thanked. This is in no particular order as I believe everyone who helped in this process was as equally important to the final outcome.

To my mother who is forever a rock in my life. She is truly inspirational.

To my supervisor Dr Julie Deslippe who was ever so patient, knowledgeable, and enthusiastic. An invaluable source of information, insurmountable eagerness and fun.

To Natascha for being an amazing lab mate who has been there over and over again willingly giving up her time to help me with lab work and theory.

To my sister Georgia. My best friend and by far the best source of knowledge when it comes to anything $\mathrm{R}$ related.

To my lab group: Nicki, Natascha, Shannon, Leslie and Stephanie thanks for being a great team. I must say we do have a lot of fun.

To my Nelson Crew, thanks for making my move to Wellington that much better. And for the adventures I was able to get out on away from university work.

To Ben, Bobby, Alex, Sean, Tmac, Dez and the rest of the university crew. Thanks for times at and away from university. Many great adventures have been had and many more to come.

To Lindie for countless hours spent checking in. I am forever grateful to you. 


\section{Table of Contents}

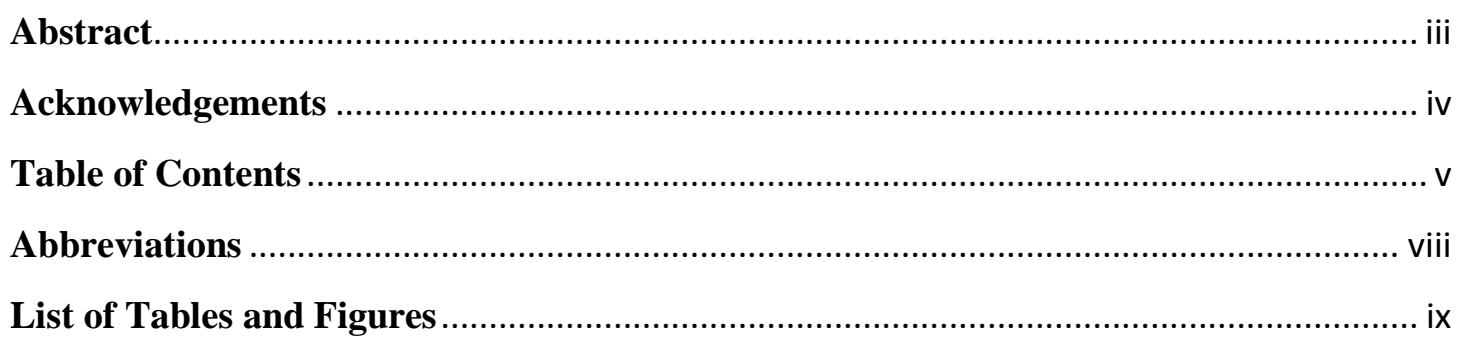

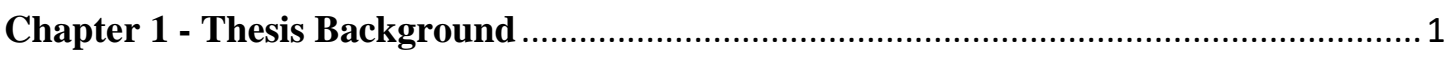

1.1 Plant community richness - Relationship between species diversity and ecosystem

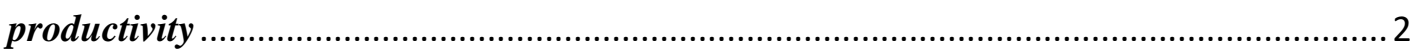

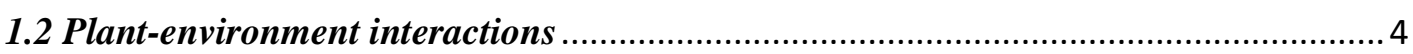

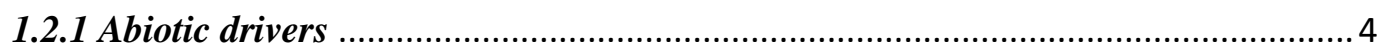

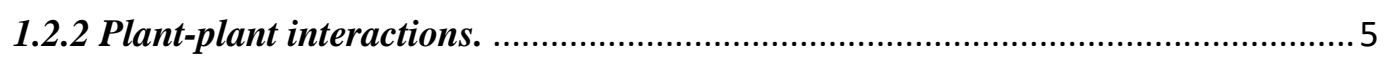

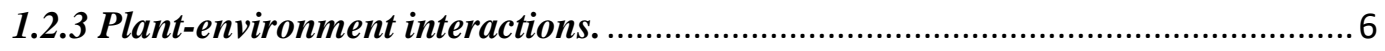

1.2.4 Plant-soil microbe interactions.........................................................................

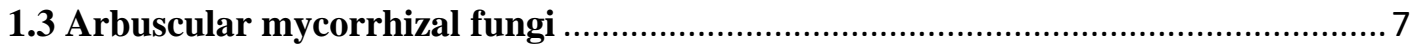

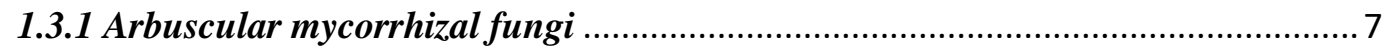

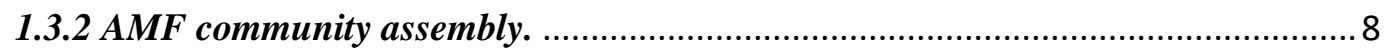

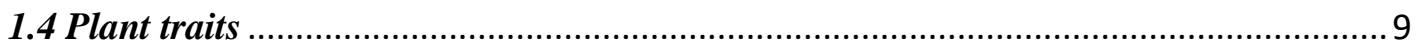

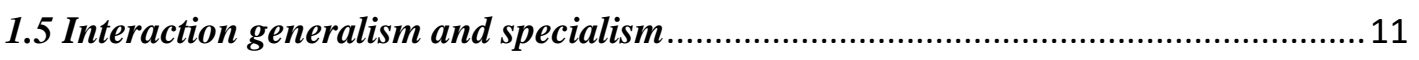

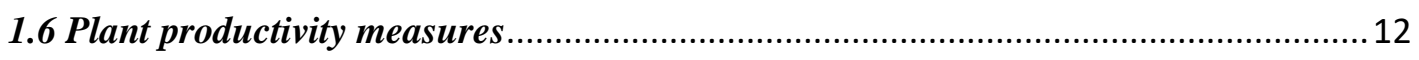

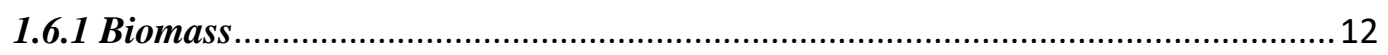

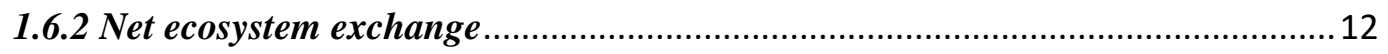

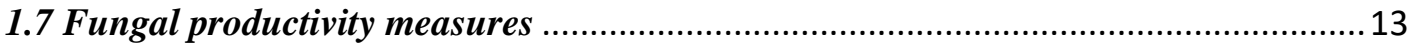

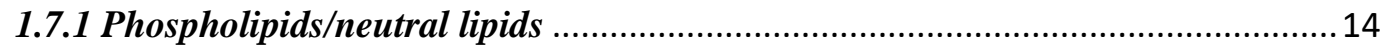

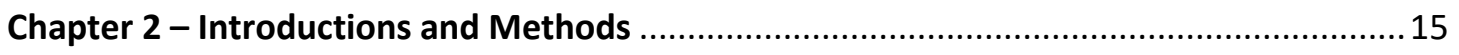

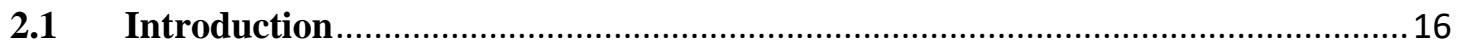

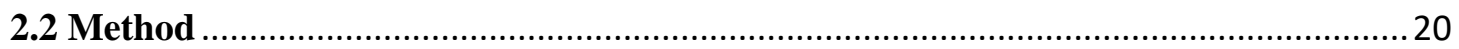

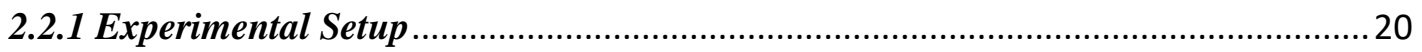

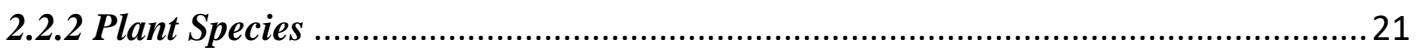




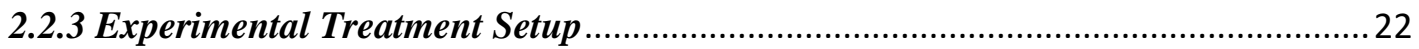

2.2.4 Productivity response to plant richness and interaction trait experiment ...............23

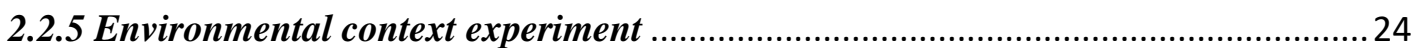

2.2.6 The Environmental context experimental setup ..................................................24

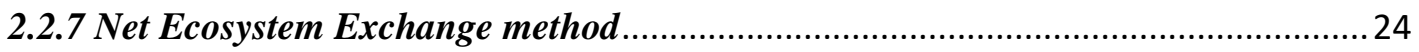

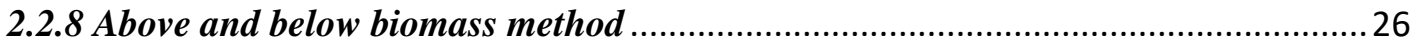

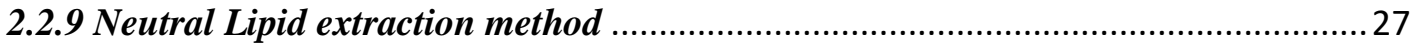

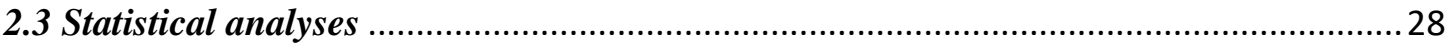

2.3.1 Productivity response to richness and interaction trait......................................29

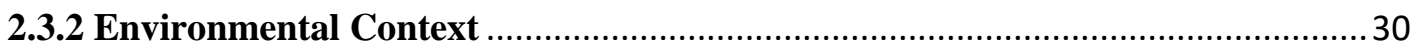

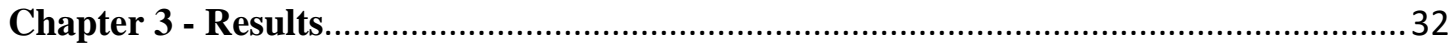

3.1 Productivity response to plant richness and interaction trait................................ 33

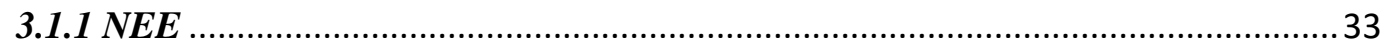

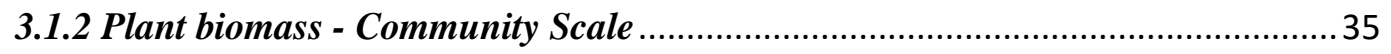

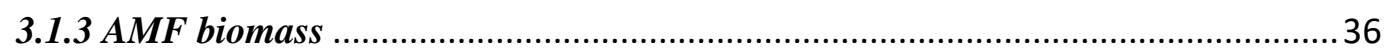

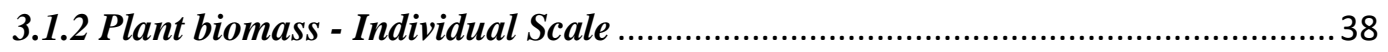

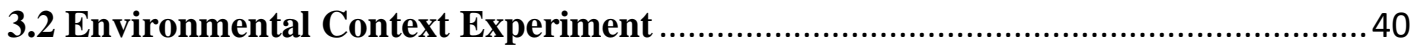

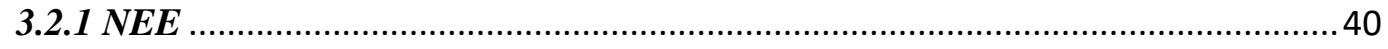

3.2.2 Plant biomass - Community Scale ………......................................................... 43

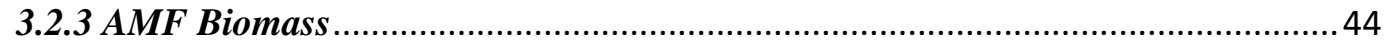

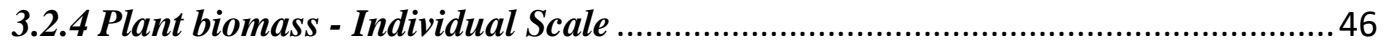

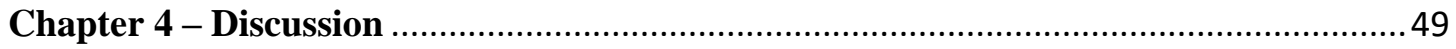

4.1 Productivity response to plant richness and interaction trait..................................5 50

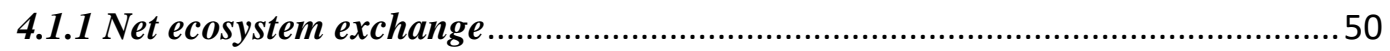

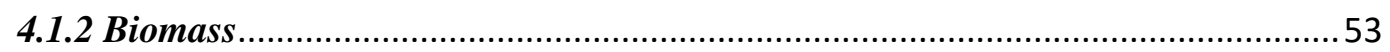

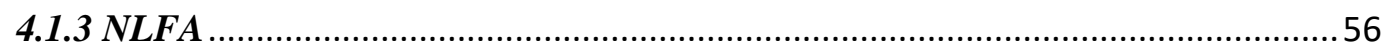

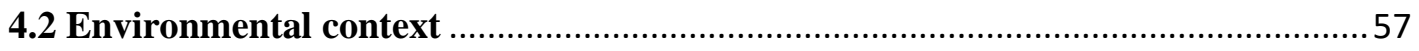

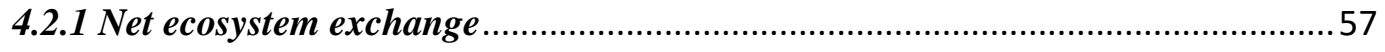

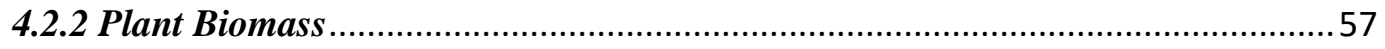

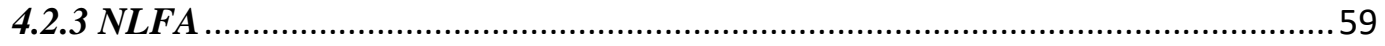

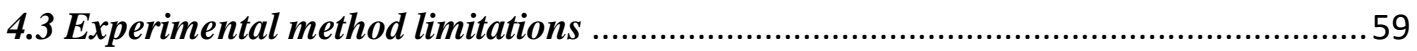




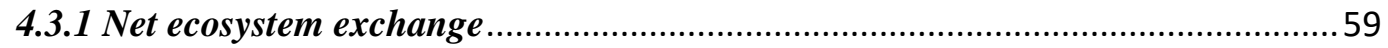

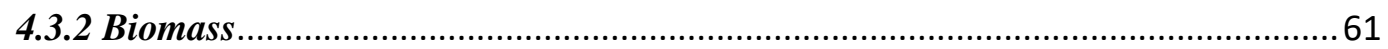

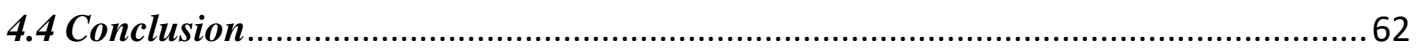

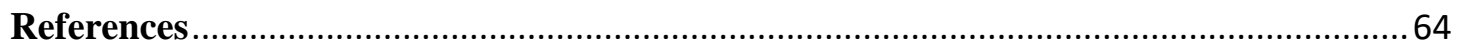




\section{Abbreviations}

$\begin{array}{ll}\text { AMF } & \text { Arbuscular mycorrhizal fungi } \\ \text { ANOVA } & \text { Analysis of variance } \\ \text { GPP } & \text { Gross primary productivity } \\ \text { NEE } & \text { Net ecosystem exchange } \\ \text { NEP } & \text { Net ecosystem productivity } \\ \text { NLFA } & \text { Neutral lipid fatty acid } \\ \text { NPP } & \text { Net primary productivity } \\ \text { PLFA } & \text { Phospholipid fatty acid } \\ \text { PRR } & \text { Productivity richness relationships }\end{array}$




\section{List of Tables and Figures}

Table 2. 1: Plant species used in this experiment. Showing family and interaction trait. The generalist rank shows G1 as most generalist and S1 as most specialist.

Figure 1. 1: Plant species ranked by interaction generality and species selection (Deslippe, Tylianakis, \& Hartmann, 2016).

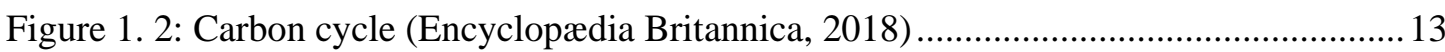

Figure 2. 1: Map of the Wairio Wetland on the eastern shore of Lake Wairarapa which is run by the conservation group Duck Unlimited and the Department of Conservation. The study site for this experiment was the area labelled Stage 3 in this figure. The red dot represents where the transect lines were taken from. https://www.ducks.org.nz/images/PDF/wairio_map3.pdf ...

Figure 2. 2: a) Experimental communities constructed for the varying species richness treatment. Initial plant communities contained either 2, 4 or 8 plant species, with 1 individual per species. b) A diagram showing the setup of all the different plant community setups grown in this experiment across all the different soil treatments (community 1,2, and 3). The green circles represent generalist species and the blue circles represent specialist species.....23 Figure 2. 3: Plastic NEE chamber with a Li-COR 7500A infrared gas analyser attached inside

Figure 3. 1: Slope of Net Ecosystem exchange in mg of carbon dioxide in meters per second. Treatment along the bottom shows the interaction trait of the late arrival plant. Runs are represented by A, B and C, where A is Run 1, B is Run 2, and C is Run 3. The columns represent the different community types with the left-hand column as community 1 , the middle column as community 2 and the right-hand column as community 3 . Significant differences between interaction traits (Tukey post hoc test, $\mathrm{p}<0.05$ ) are indicated by different lowercase letters above the boxplots.

Figure 3. 2: Total plant biomass (g) of community types 1 ( 3 plants) , 2 (5 plants) and 3 (9 plants), separated into above ground biomass (orange) and below ground biomass (blue). Bars show means $\pm \operatorname{SE}(n=5)$. Capital letters show the significant differences $(p<0.05$; Tukey post-hoc) in the below ground biomass and the lower-case letters show significant differences in both above ground biomass and total biomass.

Figure 3. 3: The concentration of 16:1w5, the NLFA biomarker for AMF, in $9.5 \mathrm{~kg}$ of growth medium (individual treatment pot volume), $\mu$ mol per Total soil per treatment pots.

Figure 3. 4: 1. The total amount of the NLFA biomarker for AMF 16:1w5 (in $\mu \mathrm{mol}$ ) per community root system per species richness treatment (3,5 or 9 plant species). Significant differences between community types (Tukey pot-hoc, $\mathrm{p}<005$ ) are shown by lower case letters. 2. The total amount of the NLFA biomarker for AMF 16:1w5 (in $\mu \mathrm{mol}$ ) per gram of root per species richness treatment (3, 5 or 9 plant species). Significant differences between community types (Tukey pot-hoc, $\mathrm{p}<005$ ) are shown by lower case letters

Figure 3. 5: Individual above ground biomass (g) for each plant species in this experiment for first arrivals. Plant species are shown here using the six-letter code. Letters 1, 2, and 3 represent the respective community treatments. 
Figure 3. 6: Individual above ground biomass (g) for each plant species in this experiment for "late arrival" plants planted into the original communities. Plant species are shown here using the six-letter code. Letters 1, 2, and 3 represent the respective community treatments. 40 Figure 3. 7: Slope of Net Ecosystem exchange (carbon draw down) in mg of carbon dioxide in meters per second. Treatment along the bottom shows the respective community being measured. Runs are represented by A, B and C, where A is Run 1, B is Run 2, and C is Run 3. The columns represent the different soil types.

Figure 3. 8: Total community biomass in $\mathrm{g}$. Above ground biomass in orange and below ground biomass in blue. Values presented are means with \pm SE $(n=5)$. Panels represents three soil conditions; normal, sterilized, and sterilized but reinoculated soil. Capital letters show the significant differences $(\mathrm{p}<0.05$; Tukey post-hoc) in the below ground biomass and the lowercase letters show significant differences in the above ground biomass.

Figure 3. 9: Total NLFA biomarker for AMF 16:1w5 (in $\mu \mathrm{mol}$ ) per pot by species richness treatment (3, 5 or 9 plant species), in each of the three soil types; normal, inoculated and sterile. Significant differences between community types (Tukey pot-hoc, $p<005$ ) are shown by lower case letters

Figure 3. 10: 1. The total amount of the NLFA biomarker for AMF 16:1w5 (in $\mu \mathrm{mol}$ ) per community root system per species richness treatment (3,5 or 9 plant species) in the three different soil types. Significant differences between community types (Tukey pot-hoc, $\mathrm{p}<005$ ) are shown by lower case letters. 2. The total amount of the NLFA biomarker for AMF 16:1w5 (in $\mu \mathrm{mol})$ per gram of root per species richness treatment (3, 5 or 9 plant species) in the three different soil types.

Figure 3. 11: Individual above ground biomass (g) for each plant species in the environmental context experiment for initial communities. The fill shows the three different soil types. Plant species are shown here using the six-letter code. Letters 1,2, and 3 represent the respective community treatments.

Figure 3. 12: Individual above ground biomass (g) for each plant species in the environmental context experiment for late arrivals. The fill shows the three different soil treatments. Plant species are shown here using the six-letter code. Letters 1, 2, and 3 represent the respective community treatments. 
Chapter 1 - Thesis Background 


\section{Thesis Background}

Ecosystem science investigates the components of an ecosystem which includes all the living organisms as well as all the non-living components that integrate with one another to give a functioning system. Energy flows from the photosynthesis of primary producers all the way through to decomposers who allow the release of carbon back into a form readily available for primary producers to use again. Within these highly diverse and fluctuating systems there are systems that function at different temporal and spatial scales. These systems can be divided into communities which are groups of populations of a specific species functioning in a certain spatial and temporal scale. Community ecology often studies the association between populations or groups that share similar spatial and/or temporal zones. This study will try to understand the complexities of plant communities with the addition of certain plant interaction traits. By manipulating plant communities and incorporating different functional groups and plant traits, relationships between plants and their below ground interaction partners on overall productivity can be assessed

\subsection{Plant community richness - Relationship between species diversity and ecosystem productivity}

Species composition and diversity effects on ecosystem functioning is an area of plant community ecology that has experienced a significant paradigm shift over the past half century. The present-day way of thinking is biodiversity is a major determinant of community and ecosystem dynamics and functioning. This paradigm was brought about in the 50's but curbed as community and ecosystem ecology moved to a more theoretical paradigm which lead researchers to believe individual species were less stable at higher biodiversity levels. This has lead research in the direction of what the effects diversity is having on the stability of natural ecosystems (Tilman, Isbell, \& Cowles, 2014). Species richness and productivity are a part of plant community ecology that has been studied with great interest. The structures and functions that drive these richness and productivity relationships are fundamental to understanding the determinants of biodiversity (Mittelbach et al., 2001). Tilman (1997) showed that plant community composition and diversity are significant determinants of ecosystems processes. He showed that the loss and addition of species with certain functional traits could have a large impact on community productivity. Community productivity and richness can be directly related with different types of relationships (Cardinale et al., 2007; Klironomos, McCune, Hart, \& Neville, 2000; Mittelbach et al., 2001; Tilman et al., 2001; Waide et al., 1999; Yachi \& Loreau, 1999). Species richness in relation to biomass has been a contentious issue with many popular views focusing on the hump shaped relationship 
(unimodal) (Guo \& Berry, 1998; Mittelbach et al., 2001). Waide et al. (1999) reviewed over 200 articles that had published empirical relationships between species richness and productivity at different spatial scale factors on species richness and productivity wanting to find whether unimodal, positive, negative or no relationship were stronger in different terrestrial biomes. Within and below spatial scales, unimodal patterns were dominant (30\%), positive next (12\%), negative was less dominant (12\%), however no relationship was overall, the most common pattern (32\%). At continental scales, there were no negative relationships but very strong positive relationships and weak unimodal relationships. In a study to test the different modes of productivity-richness relationships (PRR), Adler et al. (2011) showed there were no general relationships between productivity and richness at different spatial scales. This paper concludes that continued efforts in understanding general PRR, need to focus on investigating the complex, multivariate processes that regulate both productivity and richness. Grace et al. (2016) concluded similar results, that in different spatial scale processes over time PRR are working simultaneously as part of a whole system. These processes need to be integrated for future testing. Hector et al. (1999) showed that with a reduction in biodiversity there was a reduction in community productivity, possibly driven by reduced niche complementarity, as species were removed from the community resources were not maximally utilised (Tilman et al., 2001). Hector et al. (1999) discussed the process by which the loss of plant species richness could decrease productivity. These processes were the "sampling effect" where communities that are more diverse are more likely dominated by highly productive species. The process of niche complementarity whereby ecological differences among species allows for more complete exploitation of resources in a more diverse community relative to a depauperate community. In addition, the process of removing mutualistic interactions between species in communities with fewer species will limit production. Cheng, Zhang, Zhao, and von Gadow (2018) found that in forest stands productivity-diversity relationship is closely related to the dominance of individual species at the species level. Where the most dominant species have a negative effect on productivity as they exert greater interspecific competition and dominate less dominant species, which could negatively affect species richness by competitive exclusion.

In order to avoid interspecific competition, communities tend to be composed of species with different multidimensional niches. As proposed by the Limiting Similarity Hypothesis "traits must differ from those of its superior competitor by a finite amount" (Tilman, 1994), which can be complementary thus increasing productivity and overall community performance above that of each individual (Loreau \& Hector, 2001; Tilman et al., 2001). Niche complementary can also have negative effects on overall biomass of communities. As more species are added to a community niche overlap can occur along with interspecific 
competition, resulting in the dominance of a species with specific traits and a potential loss of biomass (Loreau \& Hector, 2001). Disentangling the complementarity effect can indicate where interspecific interactions are contributing to the overall ecosystem function or having detrimental effects or no contribution at all (Kirwan et al., 2009). Kirwan et al. (2009) created a specific interaction model, which could assess the diversity-function of a plant community using the effect of individual species interacting in that community. Tilman et al. (2014) summarised that selection effects and interspecific complementarity were two of the key driving forces behind the biodiversity-ecosystem functioning relationship. Facultative AMFplant relationships are examples of niche differentiation and complementarity that results in overall community biomass increase (Klironomos et al., 2000). Klironomos et al. (2000) tested AMF-plant relationships and found that productivity in plant communities with AMF was higher than communities without AMF. In addition, maximum plant biomass was reached at lower species richness when in the presence of AMF showing AMF can buffer the impacts of plant removals on the overall productivity of a community. Not only is richness in a community important for overall ecosystem functioning, species complementarity can play a large role in the multi functionality of a given community. Baeten et al. (2019) found that it mattered considerably which species or species pairs were promoted in a given community richness to create the highest multi-functionality. Identifying species in a community, which interact to provide the highest functionality, could lead to the optimisation of one or multiple ecosystem functions (Storkey et al., 2015). The relationship between species richness and production may not be linear because many factors that co-vary with species richness may affect ecosystem production (Li et al., 2019). Hector et al. (1999) found that biomass patterns predict a log-linear decline in productivity when there is a reduction in plant species richness, where a reduction in niche complementary or positive species interactions both appear to play a role. Mayfield et al. (2010) writes, "size of the local species pool, the productivity of the system, the type of disturbance and the traits examined are all likely to be key factors in determining the trajectory" of a change in species diversity and functional traits. When assessing overall productivity of a community the functional groups and plant traits within the community play an important role in understanding the productivity results of the community.

\subsection{Plant-environment interactions}

\subsubsection{Abiotic drivers}

As sessile organisms, higher plants must acquire water and nutrients from the environment from a position that requires a certain plasticity to overcome the inability to move with the environment. This position is in constant flux as the environment is forever changing while 
plants cannot 'move' with it. Carbon dioxide, water and nutrients are acquired from their sedentary position while they use available light to photosynthesise (De Jong \& Leyser, 2012). As light is the driving force behind plants ability to acquire energy for growth water is another important limiting resource, with which increases or decreases plants ability to survive in their environment. Lauenroth and Sala (1992) found that there was an increase in production across a precipitation gradient. Precipitation increased movement of water, which can mobilise nutrients in the soil allowing better uptake possibilities for plants (Lauenroth \& Sala, 1992). Although light drives photosynthesis, both low and high sunlight can limit plant performance. Shortages of key resources can reduce survival and growth. Where plants face heat, desiccation, excessive irradiance, and UV radiation stresses in high sunlight, to cope with these stresses requires high protective investments (Demmig-Adams \& Adams, 2006). With an increase in both anthropogenic carbon and nitrogen in the atmosphere and soil there, is a change in short-term plant growth increasing productivity and altering the plant chemistry, which can then lead to changes in biotic interactions (Hattenschwiler, 2001; J. M. Tylianakis, Didham, Bascompte, \& Wardle, 2008).

\subsubsection{Plant-plant interactions.}

The composition and structure of plant communities arise as consequences of the interactions among species as well as between plants and other trophic groups. The composition and structure of plant communities is temporally and spatially variable creating a complicated set of associations driving composition and structure (Almany, 2004; Ross \& Harper, 1972). In a review done by Callaway (1995), the idea of facilitation as a driving force behind plant community structures both temporally and spatially shed new light. Callaway (1995) observed community structure and function through the facilitation of plant-plant interactions in terms of: light and temperature, soil moisture, soil nutrients, soil oxygenation, substrate modification, protection from herbivores, pollination, and soil microbes and mycorrhiza.

Not only are plants competing for the same limiting resources, the temporal differences in plants arrival time into an environment can have profound effects on community structure and function. A priority effect occurs when the outcome of a plant-plant interaction depends on the order in which each species arrives at a site (Fukami, 2015). Priority effects on community composition is a concept that has gained a lot of traction in the literature over the past 15 years. Ross and Harper (1972) first investigated the importance of first arrivals concept. They looked at the effects of seed emergence time on species growth rates and found significant effects of plant species identity illustrating the idea that 'the early bird gets the worm' (Gioria \& Pysek, 2017). Understanding the priority effects and assembly history, or as 
Fukami (2015) describes it, "the historic contingency", that occur in terrestrial plant communities has large importance in the successional rates of a community. It also plays a large role in the productivity of a community, and the drivers of community assembly (Fukami, 2015; Fukami, Bezemer, Mortimer, \& van der Putten, 2005). The priority effects of a community can also be applied in the planning of restoration (Dickson, Hopwood, \& Wilsey, 2012; Grman \& Suding, 2010). The arrival of species and species arrival history is a biotic event, which can be subtle to observe but have large impacts on structure and function of communities both with an inhibitory or facilitative effect (Fukami, 2015). Priority effects can lead to alternative community stable states, alternative transient states, and compositional cycles (Fukami, 2015). When niche similarity occurs niche pre-emption and modification are important in the success of late arriving species. Pre-emption occurs when a species arrives first and utilises resources available to them depleting what is available for next arrivals (as shown by Ross and Harper (1972) (Fukami, 2015; Goodale \& Wilsey, 2018). Modification occurs when first arrivals alter and modify the environment making arrival for future species difficult (Fukami, 2015). Priority effects can have large impacts on the abundance of native species in the presence of an exotic species, in grasslands (Goodale \& Wilsey, 2018). Exotic species that begin growth before native plants have higher seedling growth rates, earlier emergence, and better germination rates (Goodale \& Wilsey, 2018). In grasslands, native species that are well established can maintain survival in the presence of exotic invaders (Abraham, Corbin, \& D'Antonio, 2009). If there are limiting resources native plants have shown to respond better than exotic plants (Davidson, Jennions, \& Nicotra, 2011). Native species can gain pre-emption of resources, which leads to modification of the environment giving them an advantage over exotic invaders (Vaughn \& Young, 2015).

\subsubsection{Plant-environment interactions.}

Priority effects can also be strengthened or weakened by abiotic conditions (Goodale \& Wilsey, 2018; Richards, Bossdorf, Muth, Gurevitch, \& Pigliucci, 2006). Goodale and Wilsey (2018) investigated the effects of precipitation on the strength of priority effects in exotic and native grassland ecosystems. Exotic species were found to have stronger priority effects across environmental gradients supporting that exotics have wide niche breadth and plasticity allowing them to establish and maintain dominance over native species. Priority effects are important to trace back over long evolutionary time scales to understand native ecosystems with respect to environmental change. Leopold, Tanentzap, Lee, Heenan, and Fukami (2015) investigated alpine plants in New Zealand and found evolutionary priority effects make distributions hard to understand but environmental gradients can help predict both abundance and distribution. 


\subsubsection{Plant-soil microbe interactions.}

Through the production of root exudates that are a carbon source for microbes and their modification of the structure of the soil environment, plants can also induce priority effects in soil microbial communities. These effects generate soil legacies which may persist in the system long after the plant is removed (Grman \& Suding, 2010; Pfennigwerth, Van Nuland, Bailey, \& Schweitzer, 2018; Weidlich et al., 2018). These legacies can have both positive and negative effects on the growth of later coloniser plants. Soil legacies can involve the accumulation of allelochemicals in the soil, which kill roots and plant species as well as inhibiting root interaction with mycorrhizal fungi (Dickie et al., 2017; Grman \& Suding, 2010). Positive plant-soil feedbacks are expected to promote maintenance of dominance, possibly resulting in monocultures, whereas negative plant-soil feedbacks are expected to promote species' succession (Paul Kardol, Cornips, van Kempen, Bakx-Schotman, \& van der Putten, 2007). Carey, Blankinship, Eviner, Malmstrom, and Hart (2017) found invasive grass species decreased the active micro biota biomass in the soil. Carbon in soil is important for net productivity of ecosystems and is stored in the soil by the biota in the soil, which are affected by plant community traits (De Deyn, Cornelissen, \& Bardgett, 2008; Weidlich et al., 2018). There is limited literature and understanding on the distribution of AMF in soil, which in turn has created difficulties developing theories about the roles played by soil microbes in shaping priority effects in plant-microbial communities (Dickie et al., 2017; Pfennigwerth et al., 2018; Weidlich et al., 2018).

\subsection{Arbuscular mycorrhizal fungi}

\subsubsection{Arbuscular mycorrhizal fungi}

Arbuscular mycorrhizal fungi (AMF) are global plant root symbionts that belong to the phylum Glomeromycota, which is one of 8 phyla in the kingdom Fungi (Hibbet et al 2007). AMF are obligate symbionts that invade the root cortical cells of plants forming symbiotic relationships, which allow plants to uptake poorly labile phosphate and other inorganic irons in the soil (Schüssler et al 2001). This ancient symbiosis, considered the ancestral state of land plants, was well established more than 400 million years ago and is still formed obligately by the majority of extant plant species (Redeker et al 2000). As obligate biotrophs, AMF grow only in the presence of living plants. There is discrepancy between the high diversity of plants that host AMF ( 300,000 species) and the low number of AMF taxa ( 700 species) suggesting that many of the AMF taxa are host generalists (Opeik et al 2010). AMF are generally shown to exhibit low host specificity (Smith \& Read, 2008). Although, individual AMF taxa are capable of colonising a wide range of host plants, growing evidence 
of AMF host preference is emerging (Vandenkoornhuyse et al 2007 and Gollete 2004). Rillig (2004) suggests AMF are positioned on the "abiotic/biotic interface" of ecosystems, as they are critical pathways for plant nutrient uptake, and are sensitive to global change factors like climate, agricultural practices and pollution. Therefore, insight to specific AMF host preferences and its consequences for plant communities, are likely to be increasingly important for our understanding of plant community ecology into the future.

Plants interact with a complex and changeable abiotic and biotic environment. Plant-plant, plant-herbivore and plant-climate interactions all have important effects on plant community structure and productivity at local scales. As over $80 \%$ of extant terrestrial vascular plant families form a symbiosis with AMF, these interactions are likely to be important regulators of plant community structure and productivity globally (Schüßler, Schwarzott, \& Walker, 2001). The direct and indirect mechanisms by which existing networks of plants and AMF influence incumbent plant species, both alien and native, is an area that has only recently been considered (Moora et al., 2011). Studies have found that invasive species alter the composition of AMF in the soil increasing or decreasing the AMF present (plant speciesspecific) based on functional traits of the plants (Batten, Scow, Davies, \& Harrison, 2006; Batten, Scow, \& Espeland, 2008; Greipsson \& DiTommaso, 2006; Mummey \& Rillig, 2006). Invasive plants have the ability to associate with a widespread range of AMF taxa making them successful when invading new habitats with native AMF taxa (Moora et al., 2011). AMF themselves are almost unrestricted in their ability to form successful symbioses with most plant species (Smith \& Read, 2008).

\subsubsection{AMF community assembly.}

The roles played by soil microbes in ecosystems are important but understanding their functional traits and how they influence plant communities is limited. Most studies have targeted the AMF functional traits that provide plants with nutrients. However there is a lack in understanding around functional traits of AMF in field studies (Ylva Lekberg \& Helgason, 2018). The distribution of AMF worldwide is uneven and is affected by local environmental conditions (Opik et al., 2010). Sepp et al. (2019) observed minor changes in the composition of the AMF community of a grassland when sampling at seasonal intervals and concluded that a single sampling event can be enough to describe AMF diversity. AMF taxa have restricted distributions which may be correlated to biogeographical ranges like climatic zones and continental limits and to phylogenetic limits such as uneven distribution between vascular plant suborders (Opik et al., 2010). Dominant AMF taxa colonizing plant roots may differ between habitat types, host species and locations in the root system (Opik, Moora, Liira, \& 
Zobel, 2006). Grasslands and tropical forest are shown to be AMF taxon rich habitats, where arable fields have low diversity and low taxon richness (Opik et al., 2006; Opik et al., 2008). Until recently the traditional view has been shown that AMF associate with the majority of plant species and are generally believed to exhibit low host specificity (Smith \& Read, 2008). However, this view is changing as research shows that AMF communities interact and associate with different plant roots (Mari et al., 2011). Davison et al (2011) showed a new novel way in which AMF fungal communities change during plant community assembly. Host plants can affect the composition and diversity of fungal communities due to varying life stages, which in turn can affect the AMF communities that plants host (Mari et al., 2011; Opik et al., 2006). Engelmoer and Kiers (2015) developed a study looking at the influence of the host plant on the mycorrhizal networks asking the question whether there were "effects of host diversity on the growth of an AMF network originating from two spatially separated host roots". They found that fungal abundance was three to ten times lower in mixed host setups than in comparison with monoculture setups. The science around AMF functional traits and their effects on plant growth are becoming better understood as more AMF isolates are being cultured in labs (Chagnon, Bradley, Maherali, \& Klironomos, 2013; Opik et al., 2006). AMF associations show significant changes in the host plants and their environments. Changes occur in the rhizosphere, influencing soil structure, carbon deposition, microbial communities, and influence the other plant-microbe interactions (Jung, Martinez-Medina, Lopez-Raez, \& Pozo, 2012). Chagnon et al. (2013) grouped AMF in functional groups and argues that classifying trait values gives insights into interactions between AMF-AMF traits, AMF-plant traits and environmental filters. van der Heijden, Boller, Wiemken, and Sanders (1998) showed that AMF composition and richness was an important part of plant species composition, variability, productivity and biodiversity.

\subsection{Plant traits}

Traits are "any morphological, physiological or phenological feature measurable at the individual level, from the cell to the whole-organism level, without reference to the environment or any other level of organization" (Garnier \& Navas, 2012). Plant communities are the end results of abiotic and biotic factors which act as filters on specific plant functional traits allowing only certain individuals to persist in a certain environment (Lavorel \& Garnier, 2002). An understanding of the processes by which plant traits are filtered by the environment allows insight into the mechanisms of community assembly (Garnier \& Navas, 2012). As communities form, individual plant functional traits are important in understanding how plant species can co-exist. Garnier \& Navas (2012) discuss three widely accepted dimensions that are important to plant function and ecological strategy. The resource use dimension looks at 
the ability of individuals to persist in a site due to the differences in resource acquisition. Because the acquisition of carbon controls much of the plant's downstream metabolic pathways, the functional traits of green leaves constitutes the widest range of interspecific variability across the resource use dimension (Garnier \& Navas, 2012; Lavorel \& Garnier, 2002). The height dimension is a functional trait of plants which is important in an individual's ability to gain enough light, space, reproductive potential, and seed dispersal (Garnier \& Navas, 2012). The seed size dimension is a functional trait of plants which creates a continuum of success in rates of offspring survival, due to differences in seed sizes and seed quantity. The seed size dimension is a functional trait of plants, which creates a continuum of success in rates of offspring survival, due to differences in seed sizes and seed quantity. As seeds get larger dispersal becomes harder while survival chances increase whereas as seeds get smaller dispersal is easier, expense in production is lessened however chance of survival is decrease (Garnier \& Navas, 2012). This functional continuum has parallels with the different selection strategies in some animals which invest in either r-strategies or kreproductive strategies. Like increased seed size an increase in body size can reduce environmental resistance with fewer potential predators as well as being better shielded from changes in their physical environment (Pianka, 1970).

Functional diversity is "the value and range of the functional traits of an organism in a given ecosystem" (Tilman, 2001). This can be disentangled more in respect to the indicators of these values in a community or ecosystem. Mason, Mouillot, Lee, and Wilson (2005) discuss these indices as functional characters of plant species, complexity of food webs and the total conglomeration of functional groups present. Assessing these trait values across community structures involves understanding the overall functional composition. These are defined using community weighted trait mean (Duarte, Debastiani, Carlucci, \& Diniz-Filho, 2018). Community-weighted trait means use both information of species functional traits and their distribution to understand species distribution within a metacommunity (Duarte et al., 2018). The issue with mean trait values for a species is it may not adequately represent the mean trait values for all populations or individuals of that species (Hulshof \& Swenson, 2010).

When trying to assess functional diversity and composition there is the issue that even though the difference between species is obvious and easy to measure, there is also intraspecific variability, which must be taken into account. Hulshof and Swenson (2010) were interested in trying to understand where the variation in community plant traits come from. Whether it was due to differences between species, between individuals of the same species, within individuals or within plant organs such as leaves. They found that leaf variation within 10 
species was greatest between species and least between individual leaves supporting the idea species can be differentiated by functional traits.

\subsection{Interaction generalism and specialism}

As the knowledge grows on functional interactions between plant roots and AMF fungi, plant host interaction traits, their specificity or generality for AMF, may become a useful concept in our understanding of plant community assembly. The idea of a preferential association between AMF and plant species has yet to be resolved but has led to the idea that matching both plant and AMF functional traits which maximise mutual benefits could help resolve this (Chagnon et al., 2013). There are distinct AMF communities at local scales, where certain plants benefit more depending on their responses to the local AMF communities (Klironomos, 2002, 2003; Klironomos et al., 2000). Plants have the ability to be interaction specialists or interaction generalist forming mutualistic associations with either many AMF taxa or few AMF taxa (J. Tylianakis, Martínez-García, Richardson, Peltzer, \& Dickie, 2018). Klironomos (2002) tested the growth of rare and invasive plants in soils with AMF isolated from soils of the same plants and soils from different host plants. The results showed for both invasive and rare plants there was a more positive feedback when grown in soils that had a history of the same plant species. Figure 1.1 gives a diagrammatic representation of the generalist-specialist interaction spectrum with generalist plants hosting more AMF and specialists at the other end of the spectrum hosting fewer AMF. Opik, Metsis, Daniell, Zobel, and Moora (2009) found that host plants with wide ranges associated with AMF with wide ranges and AMF fungi that are less common were more likely to associate with host plants that have narrower ranges. Sepp et al. (2019) wanted to establish whether plant species exhibit specificity towards their fungal symbionts, and whether such specificity depends on plant traits. They found that plants at different functional group levels exhibited different AM fungal richness's and locally abundant plant species exhibited richer AM fungal communities. Less abundant plants showed more specialisation in their partner associations (Figure 1).

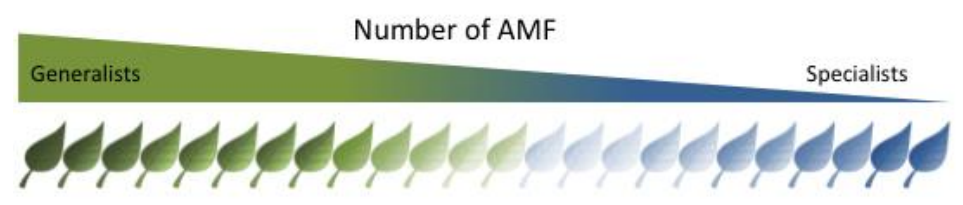

Figure 1. 1: Plant species ranked by interaction generality and species selection (Deslippe, Tylianakis, \& Hartmann, 2016). 


\subsection{Plant productivity measures}

Plant productivity can be measured in different ways. All productivity measures are representative of the overall Net primary productivity (NPP), how much carbon dioxide vegetation takes in during photosynthesis minus how much carbon dioxide the plants release during respiration, of a community.

\subsubsection{Biomass}

As a proxy for community NPP, biomass measurements of a community can be a way to do achieve this. Above ground, below ground and total plant biomass may be used to assess the productivity of a plant community. In a series of classic experiments, Tilman (1995) harvested all above ground biomass of vegetation from experimental communities $(4 \times 4 \mathrm{~m}$ or 9X9m plots in a field experiment) and used this "community biomass", which was approximately proportional to aboveground primary productivity, as a primary response variable to experimental treatments. The calculation of net primary production that is reported in most temperate forests is based on aboveground biomass only (Waide et al., 1999). Both Kajimoto, Osawa, Usol'tsev, and P. Abaimov (2010) and Kamruzzaman, Ahmed, and Osawa (2017) used the aboveground biomass (stem, branch, leaf biomass and leaf litter) of larch and mangrove forests, respectively, to calculate aboveground net primary productivity of the ecosystem. These studies illustrate how biomass can be used across a range of geographical scales to calculate productivity and net primary productivity in a community at the plot or stand scale.

\subsubsection{Net ecosystem exchange}

The total amount of carbon gained in photosynthesis in daylight, is considered gross primary production (GPP) (Fig. 2). This newly acquired plant carbon may be quickly lost from the system via plant respiration or retained or acquired by the soil community, via root exudation and microbe uptake (e.g., arbuscular mycorrhizal fungi) (Figure 1.2), where it may be again lost through heterotrophic respiration, or retained in microbial biomass. NPP is the net carbon gain by vegetation and is calculated as the GPP minus plant respiration. Net primary production (NPP) includes not just aboveground and belowground biomass but also the carbon that ends up in the soil (through litterfall), and the carbon transferred to microbes and carbon lost from leaves to the atmosphere as volatile emissions. This gives the resulting overall carbon left in an ecosystem which can then be used as a measure of ecosystem productivity. In most studies NPP is calculated as plant biomass only so underestimates NPP 
by as much as $30 \%$ (Catovsky, Bradford, \& Hector, 2002). Net ecosystem productivity (NEP) is inclusive of all ecosystem functions so integrates not just plant respiration but also heterotroph respiration, disturbance loss (fires and harvest) and leaching (through groundwater). Net ecosystem exchange (NEE) is the total carbon flux through an ecosystem (GPP minus ecosystem respiration excluding leaching) which gives the most accurate measure of GPP in an ecosystem (Chapin et al 2002). Breza, Souza, Sanders, and Classen (2012) showed that NEE had a strong linear relationship with productivity and vice versa. Plants with higher productivity had greater NEE.

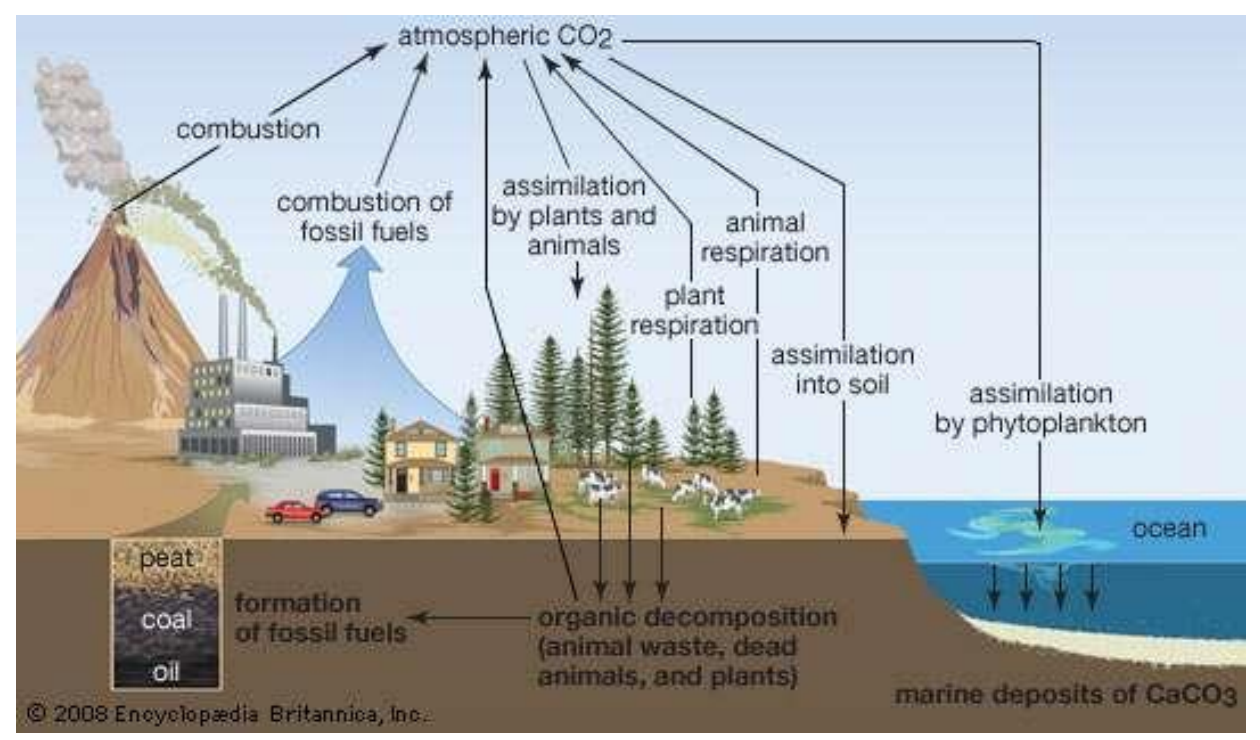

Figure 1. 2: Carbon cycle (Encyclopædia Britannica, 2018)

\subsection{Fungal productivity measures}

Measuring the plant-fungi communities can be challenging as these are ever changing (Mukerji et al 2006). Root length colonisation is a functional trait of plants that is quantified as an index of plant colonisation by AMF. The fungi communities in soil ecosystems are also important to understand as specific AMF plants prefer to host certain AMF and have different hosting abilities.

The productivity of fungi or the living biomass of fungi in the soil and roots can be measured in different ways. Counts of spores and hyphal lengths by microscopy are commonly used direct methods for the estimation of fungal biomass in roots and soils. However, these methods have been criticised for their lack of systematic or functional separation of fungal taxa, for the difficulty distinguishing dead from live materials and active from inactive 
biomass, and how time consuming they are (Balser, Treseder, \& Ekenler, 2005; Frostegard \& Baath, 1996; van der Wal et al., 2006). A major criticism of spore analysis is that Glomeromycotan fungi vary in the degree to which they form spores. Spore communities in the soil may not reflect the community of fungi active within plant roots (Antoninka, Reich, \& Johnson, 2011).

\subsubsection{Phospholipids/neutral lipids}

One method which has become popular because it avoids some of these problems metioned above is the quantification of signature lipid biomarkers for fungi. Especially useful is a fatty acid that is unique to the Phylum Glomeromycota, which enables the quantification of AMF biomass in root and soil samples. Phospholipids (PL) and neutral (NL) lipids are two fatty acids found in the membranes of soil microbes. The spores, vesicles and hyphae in AMF have a wealth of fatty acids (Vestberg et al., 2012). Phospholipids make up the lipid bilayers found in cell membranes and neutral lipids (triglycerides) are used for fat storage. Microbial lipids have become commonly used biomarkers for microbial community structure and biomass (Balser et al., 2005; Frostegard \& Baath, 1996; Willers, van Rensburg, \& Claassens, 2015). Different microbes have different dominant fatty acids, which can be used to distinguish

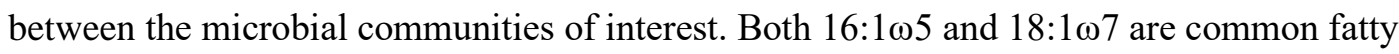
acids found in AMF that are commonly used to assess the community structure and abundance of AMF in plant communities (Johansen, Finlay, \& Olsson, 1996; Olsson, 1999). Olsson, Baath, and Jakobsen (1997) found higher concentrations of NLFA 16:1 $\omega 5$ in spores and higher concentrations of PLFA 16:105 in hyphae reflecting the difference in AMF structures but also in species. Sharma and Buyer (2015) investigated the different biochemical methods used for quantification of AMF in soil and roots, finding NLFA 16:1 15 had high correlation with spore density. Ester-linked fatty acids 16:105 had high correlation in roots when looking at colonisation and PLFA 16:1 15 had high correlation with fungal hyphal density. The use of fatty acids as a way to determine biomass are a much more repeatable and quantifiable method for fungal biomass calculations and can be considered an indicator of biomass in fungal communities. 
Chapter 2 - Introduction and Methods 


\subsection{Introduction}

The relationship between plant species richness and productivity has and continues to be a source of much debate within the ecological community. Many studies show an increase in species productivity when there is an increase in richness, however, the relationship tends to become negative when productivity exceeds a certain threshold. There are many other productivity-richness relationships (PRR) (unimodal, negative, positive) that occur more infrequently in ecosystems however these are hard to disentangle. Recent studies have stressed the importance of spatial scale and intra-process relationships in shaping PRR.

The symbiosis between plants and AMF is among the most prevalent on earth being formed obligate by the majority of extant land plant species (Redecker et al., 2013). Their ubiquity leads to complex belowground networks of plant roots linked by the mycelium of AMF species. Arbuscular mycorrhizal fungi are obligate plant symbionts that invade the root cortical cells forming a symbiotic relationship that enhances plant uptake of poorly labile phosphate and other nutrients in the soil while receiving carbon for growth (Schüßler, Schwarzott, \& Walker, 2001). These plant-AMF networks can affect communities, both through direct (e.g. competition for space, recruitment and nutrients) and indirect mechanisms (Moora et al., 2011). Through these direct and indirect mechanisms, the success of both individual plants and communities of plants are influenced by symbiosis with AMF. The effects of AMF on productivity at the individual and community scale can be assessed through measurements of productivity.

The functional traits of plants and AMF species can have a significant effect on the assembly and productivity of the plant community as a whole. Plant communities are the product of arrival and recruitment to new habitats through abiotic and biotic filters. The abiotic filters which influence plant recruitment include light, precipitation and nutrients. These filters act on specific functional traits of plant and fungal species, allowing only a subset of species to persist in a given environment (Lavorel \& Garnier, 2002). Dominant grassland species are found to have wide niche breaths and plasticity allowing them to establish and maintain dominance over non-grass species as their tolerance of changing abiotic filters is much higher than those of more specialist native plants (Goodale \& Wilsey, 2018).

Biotic filters to plant recruitment may include the availability of a symbiotic partner. Given that $>300,000$ species of plant form obligate symbiosis with $~ 700$ species of AMF, the traditional view held is that AMF exhibit low host specificity (Smith \& Read, 2008). Despite this, species-specific AMF assemblages exist among co-occurring plant species (Davidson, Jennions, \& Nicotra, 2011; Opik, Metsis, Daniell, Zobel, \& Moora, 2009). Likewise, co- 
occurring plants vary in the number of AMF taxa they host, suggesting that plants may be considered either interaction specialists or interaction generalists with regard to the amount of AMF taxa they host (Tylianakis, Martínez-García, Richardson, Peltzer, \& Dickie, 2018).

The productivity of plant communities can be influenced by priority effects within the community which occurs when the outcome of a plant-plant interaction depends on the order in which each species arrives at a site (Fukami, 2015). Priority effects that occur in terrestrial plant communities are important in the assembly, succession and productivity of a community (Fukami, 2015; Fukami, Bezemer, Mortimer, \& van der Putten, 2005). Niche partitioning in plants can be driven by priority effects and can affect overall community richness. When niche similarity occurs niche pre-emption (first arrival utilises resources) and modification (first arrivals modify their environment) are important in the success of a species (Fukami, 2015; Goodale \& Wilsey, 2018). As niche partitioning increases, the species richness in a community can also increase as a greater proportion of the total niche space is occupied. The Limiting Similarity Hypothesis states "traits must differ from those of its superior competitor by a finite amount" to survive (Tilman, 1994), which can be complementary thus increasing productivity and overall community performance above that of each individual (Loreau \& Hector, 2001; Tilman et al., 2001). All plants require a similar set of resources and have to acquire them in similar ways. Water, $\mathrm{CO}_{2}$, light, nitrogen, phosphorus, potassium and other mineral nutrients are all requirements for the growth of each plant (Silvertown, 2004). Light gradients and hydrological gradients are both process by which plant functional traits (rooting depth and leaf area) are important to allow for niche partitioning to ensure better survival along certain axes (García-Baquero, Silvertown, Gowing, \& Valle, 2016; Hulshof \& Swenson, 2010; Silvertown, 2004). Differences in functional traits among species allows for niche partitioning to occur. For example, the functional traits of roots include, depth, length, nutrient uptake ability, respiration, diameter and biotic interaction traits (Bardgett, Mommer, $\&$ De Vries, 2014). Symbiotic partner interactions in plant functional groups, such as forbs and grasses, can exhibit different root colonization rates and mycorrhizal growth responses leading to changes in productivity in these groups (Sepp et al., 2019).

Priority effects in plants effect not only plant communities but also AMF communities. Plants interaction traits exist on a spectrum of interaction generalist to specialist, forming mutualistic associations with many AMF taxa or few AMF taxa (Tylianakis et al., 2018). Our understanding of the distribution of AMF taxa in soil is limited, which in turn limits theory pertaining to the roles played by soil microbes in shaping priority effects in plant-microbial communities and their consequences for community productivity (Dickie et al., 2017; 
Klironomos et al., 2011; Pfennigwerth, Van Nuland, Bailey, \& Schweitzer, 2018; Weidlich et al., 2018; Werner \& Kiers, 2015).

Knowledge gap and thesis aims: Species composition is a strong determinant of production at the community scale. Interactions among plants, which determine the final community assembly, can be driven by priority effects. In this way, priority effects can directly influence the productivity of ecosystems (Catovsky et al., 2002). Therefore, plant interaction generality may influence the productivity of ecosystems. When interaction generalists arrive to an established community, they may compete for interaction partners. If this competition is strong it may lead to the reduction of both the biodiversity and productivity of the community. Conversely, when specialists arrive into an established community, they occupy a smaller niche space in the AMF dimension, and only require a few AMF species interactions to successfully integrate into the community. Therefore, the process of niche partitioning may allow them to recruit without the loss of other species so that biodiversity and productivity can simultaneously increase.

The aim of this experiment is to test whether the addition of interaction specialist and generalist plants to complex communities' results in different productivity of the community. I assessed productivity through several metrics:

- Net Ecosystem Exchange - net carbon assimilation by the community (respirationphotosynthesis)

- Above and below ground plant biomass of individual plants and community means.

- A neutral lipid fatty acid 16:1w5 a biomarker for arbuscular mycorrhizal fungi, was quantified in both plant roots and soil as a proxy for fungal biomass.

Because the effect of plant interaction traits on the productivity of plant communities is likely vary depending on the availability of niche space, I examined the effect of adding generalist and specialist plant species over a gradient of plant species richness. I hypothesise $\left(\mathrm{Hyp}_{1}\right)$ that the addition of an interaction specialist will increase the productivity of a plant community relative to an interaction generalist in species rich communities, but that specialists would have equivalent or lesser effects on increasing productivity than generalists at lower levels of species richness. I also hypothesise $\left(\mathrm{Hyp}_{2}\right)$ that increased plant richness will be associated with increased net ecosystem exchange, increased above and below ground plant biomass, and increased biomass of arbuscular mycorrhizal fungi.

In a second experiment, I test the effects of soil sterilisation and re-inoculation on the productivity of plant communities relative to the normal soil. I hypothesise $\left(\mathrm{Hyp}_{3}\right)$ that soils 
that have been sterilised will support lower productivity than normal soils due to the removal of the fungal community. And that communities grown in soils that are sterilised but reinoculated with AMF will partially recover the lost production. 


\subsection{Method}

\subsubsection{Experimental Setup}

The soil used in this study was field collected from Wairio Wetland on the eastern shore of Lake Wairarapa, situated on the lower North Island of New Zealand (Figure 2.1). Wairio wetland was formerly managed by a dairy corporation as rough pasture for cattle. In 2007, its management was conceded to a care group, it was subsequently fenced to exclude livestock. At the time of soil collection, the site remained dominated by a mixture of pasture grasses and exotic weeds.

The locations of soil collections were selected randomly. This was achieved by starting at a point of origin (marked by a red dot in Figure 2.1), and randomly selecting a bearing between $180^{\circ}$ and $270^{\circ}$ (roughly parallel to the lake shore). A random number between 5-50 was then selected to determine the number of steps taken from the origin to the first sampling position. This was repeated five times along the transect. Randomly selected sampling positions that were below the water table were excluded and the nearest point to the position that was above the water table was selected in its place. At each sampling position, vegetation was removed, and two 60 -litre pails of soil were collected with a shovel from the surface $40 \mathrm{~cm}$ of soil. The surface soil was selected to maximize the number of arbuscular mycorrhizal fungal spores and hyphal fragments in the soil. Three replicate transects, with five sampling positions each were applied over three days (over a period of $\sim 1$ week) until sufficient soil was collected for the glass house experiments.

Soil was then taken to the School of Biological Sciences glasshouses at Victoria University of Wellington. It was spread out on a tarpaulin to air dry and mixed by shovel prior to sieving through a $4 \mathrm{~mm}$ sieve to remove coarse fragments and to create a consistent texture. The sieved soil was then mixed with a combination of coarse and fine sand. The resultant growth medium had a 1:5 ratio of soil to sand. Sand was a dominant component of the growth medium because it enables easier harvest and cleaning of plant roots than organic constituents, but still supports good plant growth. 


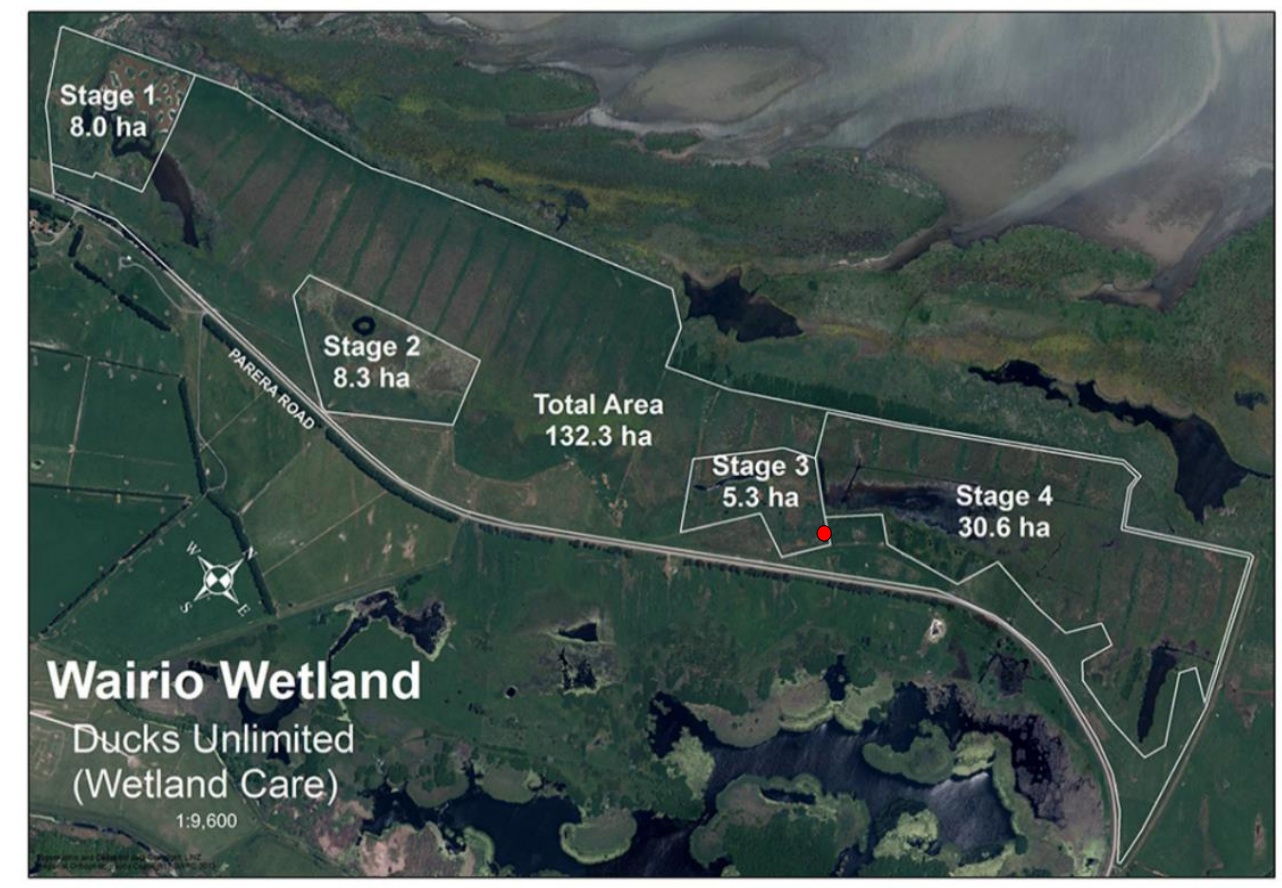

Figure 2. 1: Map of the Wairio Wetland on the eastern shore of Lake Wairarapa which is run by the conservation group Duck Unlimited and the Department of Conservation. The study site for this experiment was the area labelled Stage 3 in this figure. The red dot represents where the transect lines were taken from.

https://www.ducks.org.nz/images/PDF/wairio_map3.pdf

\subsubsection{Plant Species}

Plants selected for these experiments were chosen on the basis of previous experimental work. Briefly, field soil and seeds from 20 plant species were collected at Wairio wetland. Seed was surface-sterilized and germinated aseptically. One seedling per pot was grown in homogenised field-collected soil for 140 days before all plants were destructively harvested. There were 5 replicate plants per species. DNA was extracted from clean plant roots and fungal ITS genes were amplified and sequenced to identify arbuscular mycorrhizal fungal taxa. This yielded a range of AMF taxa per plant individual, from which alpha diversity per plant species was calculated. In general, members of the Poaceae were generalists, harbouring many AMF taxa, while forbs were specialists, harbouring relatively few AMF.

For this study, four generalist and four specialist plant species were selected, however, to ensure that patterns of plant interaction generalism could be tested over a wide taxonomic breath, selected plants were forbs and some grasses in each category. All plants were from three families, the Poaceae, Plantaginaceae and Asteraceae (Table 2.1). The four generalist 
species chosen were (from most generalist to least generalist, Holcus lanatus (G1) (Poaceae), Agrostis capillaris (G2) (Poaceae), Bromus willdenowii (G3) (Poaceae), and Plantago lanceolate (G4) (Plantaginaceae). The four specialist species chosen were from the Poaceae and Asteraceae family. The species chosen (from most specialist to least specialist) were Achillea millefolium (S1) (Asteraceae), Cichorium intybus (S2) (Asteraceae), Poa cita (S3) (Poaceae), and Schedonorus arundinaceus (S4) (Poacea) (Table 2.1).

Table 2. 1: Plant species used in this experiment. Showing family and interaction trait. The generalist rank shows G1 as most generalist and S1 as most specialist.

\begin{tabular}{|l|l|l|l|l|}
\hline Plant species & Code & Family & Interaction Trait & $\begin{array}{l}\text { Generalist } \\
\text { rank }\end{array}$ \\
\hline Holcus lanatus & HOLLAN & Poaceae & Generalist & G1 \\
\hline Agrostis capillaris & AGRCAP & Poaceae & Generalist & G2 \\
\hline Bromus willdenowii & BROWIL & Poaceae & Generalist & G3 \\
\hline Plantago lanceolate & PLALAN & Plantaginaceae & Generalist & G4 \\
\hline Achillea millefolium & ACHMIL & Asteraceae & Specialist & S1 \\
\hline Cichorium intybus & CICINT & Asteraceae & Specialist & S2 \\
\hline Poa cita & POACIT & Poaceae & Specialist & $\mathrm{S} 3$ \\
\hline $\begin{array}{l}\text { Schedonorus } \\
\text { arundinaceus }\end{array}$ & SCHARU & Poaceae & Specialist & $\mathrm{S} 4$ \\
\hline
\end{tabular}

\subsubsection{Experimental Treatment Setup}

Seeds from each specialist and generalist plant species were germinated aseptically on petri plates. Preliminary germination trails were conducted so that all seeds could be germinated on a schedule that would allow them to attain approximately the same maturity on the selected planting date (i.e. day 1 of the experiment). Seeds were transplanted into 9L pots containing 7 litres of the sand/soil growth medium when their first true leaf was present.

Seedlings were transplanted into pots to create three experimental plant communities of varying richnesses, either 2, 4 or 8 plant species. The three community's contained: 1) Two plants -G1 (Holcus lanatus) and S1 (Achillea millefolium), 2) Four plants - alternating G1, S1, G2 (Agrostis capillaris), S2 (Cichorium intybus), 3) Eight plants - alternating G1, S1, G2, S2, G3 (Bromus willdenowii), S3 (Poa cita), G4 (Plantago lanceolate), S4 (Schedonorus arundinaceus). A specialist and a generalist plant were always planted alternately to one 
another (Figure 2.2). These initial plant communities were grown for 38 days to allow plant to interact with the natural AMF inoculum in the soil. Then, one "late arrival" plant, either G1, G2, G3, S1, S2, S3 was planted into the centre of the community. The resulting community was allowed to grow for another 50 days. An automatic watering system ensured all pots were watered twice a day; in the morning and in the afternoon.

a)
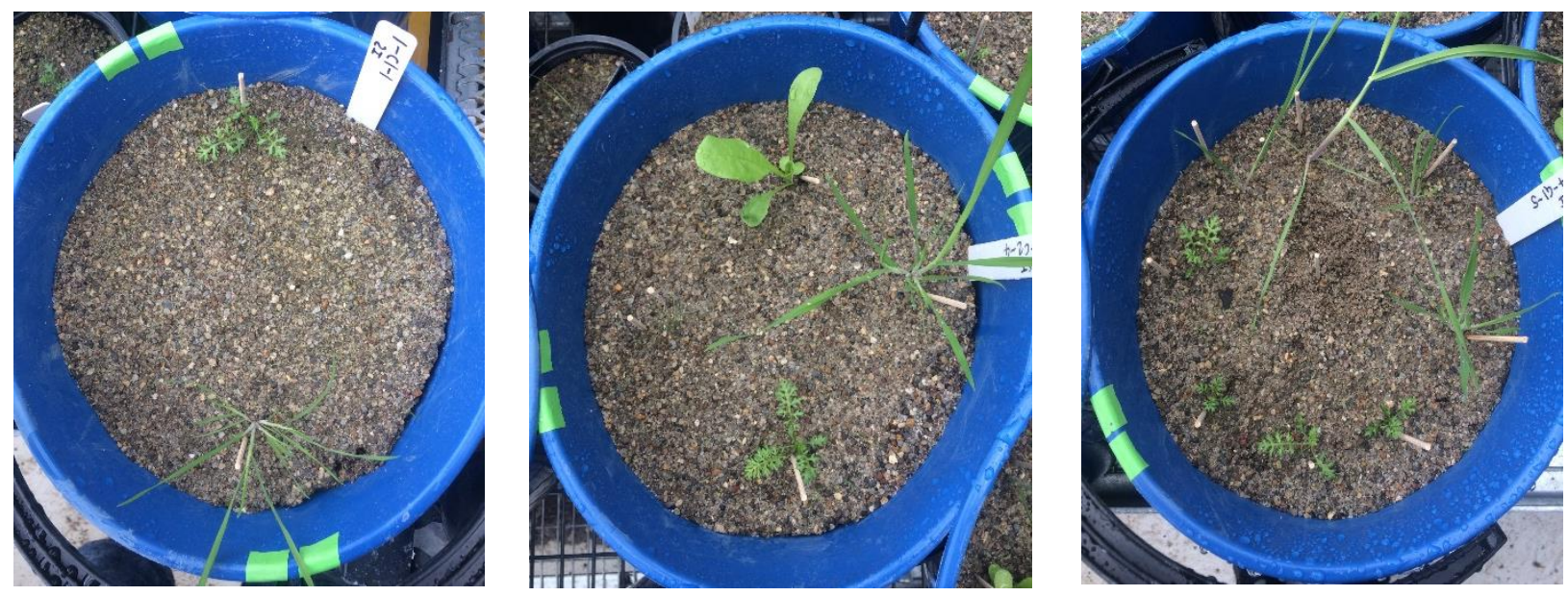

b)

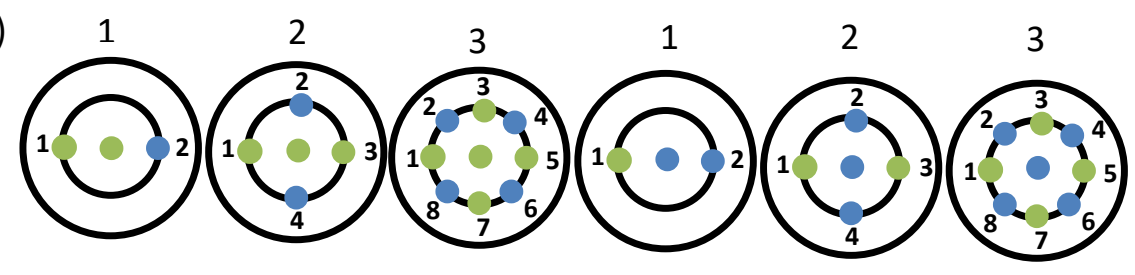

Figure 2. 2: a) Experimental communities constructed for the varying species richness treatment. Initial plant communities contained either 2, 4 or 8 plant species, with 1 individual per species. b) A diagram showing the setup of all the different plant community setups grown in this experiment across all the different soil treatments (community 1,2, and 3). The green circles represent generalist species and the blue circles represent specialist species.

\subsubsection{Productivity response to plant richness and interaction trait experiment}

All treatments in this experiment contained the full experimental setup but only used unsterilised soil and sand in the growth medium. For this experiment all treatments (pots) were replicated 5 times with a total 105 treatment pots in this experiment. 


\subsubsection{Environmental context experiment}

In order to be able to parse the effects of plant niche-partitioning for interaction partners from those due to niche-partitioning for other resources (e.g. soil nutrients). I conducted an experiment with two control soil types. In these experiments soil, two control growth mediums were established:

1) Soil was sterilised to remove all viable AMF. Under this condition only niche partitioning for nutrients occurred.

2) Soil was sterilised to remove all viable AMF; then, sterile soil was reinoculated with viable field collected soil.

The sterilisation process took place in an autoclave with $5-10 \mathrm{~kg}$ amounts of soil put through a liquid waste cycle at $103.4 \mathrm{kPa}$ and $121{ }^{\circ} \mathrm{C}$ for $1 \mathrm{~h}$, incubated $24 \mathrm{~h}$, and autoclaved an additional $1 \mathrm{~h}$. For the re-inoculated soil treatments, $750 \mathrm{ml}$ of viable soil was added back into the seven litres of sand/soil mixture and coarsely mixed by hand prior to planting.

\subsubsection{The Environmental context experimental setup}

The Environmental context experiment setup was much the same as the Productivity response experiment however only G1 and S1 were used as late arrival plants. The treatment pots in the Productivity response experiment with just G1 and S1 plants planted into the middle of the pot were also used in the Environmental context experiment. The sterile and inoculated soil were mixed with the same soil to sand ratio and treatment pots with the three community types were planted out with either S1 or G1 added to the middle of the pot after the first arrival communities had grown for 38 days. This experiment was treated exactly the same way as the Productivity response to pant richness and interaction trait experiment. For the sterilised soils and inoculated soils only generalist and specialist one was used as the "late arrivals" as this was good enough to see the effects the two control soil treatments wold have on productivity of the three different communities without making the experiment too big. Again, all treatment pots were replicated 5 times with 135 treatment pots in total.

\subsubsection{Net Ecosystem Exchange method}

Net ecosystem exchange was measured for each experimental pot. Net ecosystem exchange (NEE) is the total carbon flux through an ecosystem (gross primary productivity minus ecosystem respiration excluding leaching) (Chapin iii, A., \& A., 2002) and is used as a measure of productivity in an ecosystem. To measure the NEE, each pot containing an experimental community was placed into a clear plastic chamber $\left(0.2925 \mathrm{~m}^{3}\right)$ containing a Li- 
COR 7500A infrared gas analyser (Figure 2.3). LED lights provided a constant photosynthetically active radiation (PAR) of $1200 \mu \mathrm{mol} \mathrm{m}{ }^{-2} \mathrm{~s}^{-1}$. The LED lights were set up with a dimmer function so over a measuring period the lights could be increased or decreased in intensity according to the natural light outside of the box. The $\mathrm{CO}_{2}$ drawn down by each plant community was measured over a 90 second period. After an initial 40 second stabilisation period, the $\mathrm{CO}_{2}$ concentration measured between 40 and 90 seconds was used to calculate a linear slope of NEE in umol $/ \mathrm{m}^{3} / \mathrm{s}$. This was then multiplied by the volume of the chamber divided by the area of the chamber floor to give the concentration of $\mathrm{CO}_{2}$ in umol $/ \mathrm{m}^{2} / \mathrm{s}$, umol was then converted into $\mathrm{mg} / \mathrm{m}^{2} / \mathrm{s}$ to give the overall concentration of the NEE measurement. The temperature in the NEE chamber was recorded at the start and end of every measurement using a digital thermometer. An extraction fan was placed inside the NEE chamber to expel warm air each NEE run. The range of temperatures during which NEE was recorded throughout this experiment ranged from $19^{\circ} \mathrm{C}-29^{\circ} \mathrm{C}$. All NEE measurements were taken from within the glasshouse between the hours of $11 \mathrm{am}$ and $4 \mathrm{pm}$ on clear days to ensure maximum photosynthetic activity.

NEE was measured at three time points. Time zero will be referred to as the time the middle plants were added to their respective communities. The first run of NEE measurements was recorded on week two, one week after the late arrival (middle) plants were added (Run1). The second run of NEE measurements were recorded 4 weeks after time zero (Run2). And the third run of NEE measurements were recorded 7 weeks after time zero (Run3). 


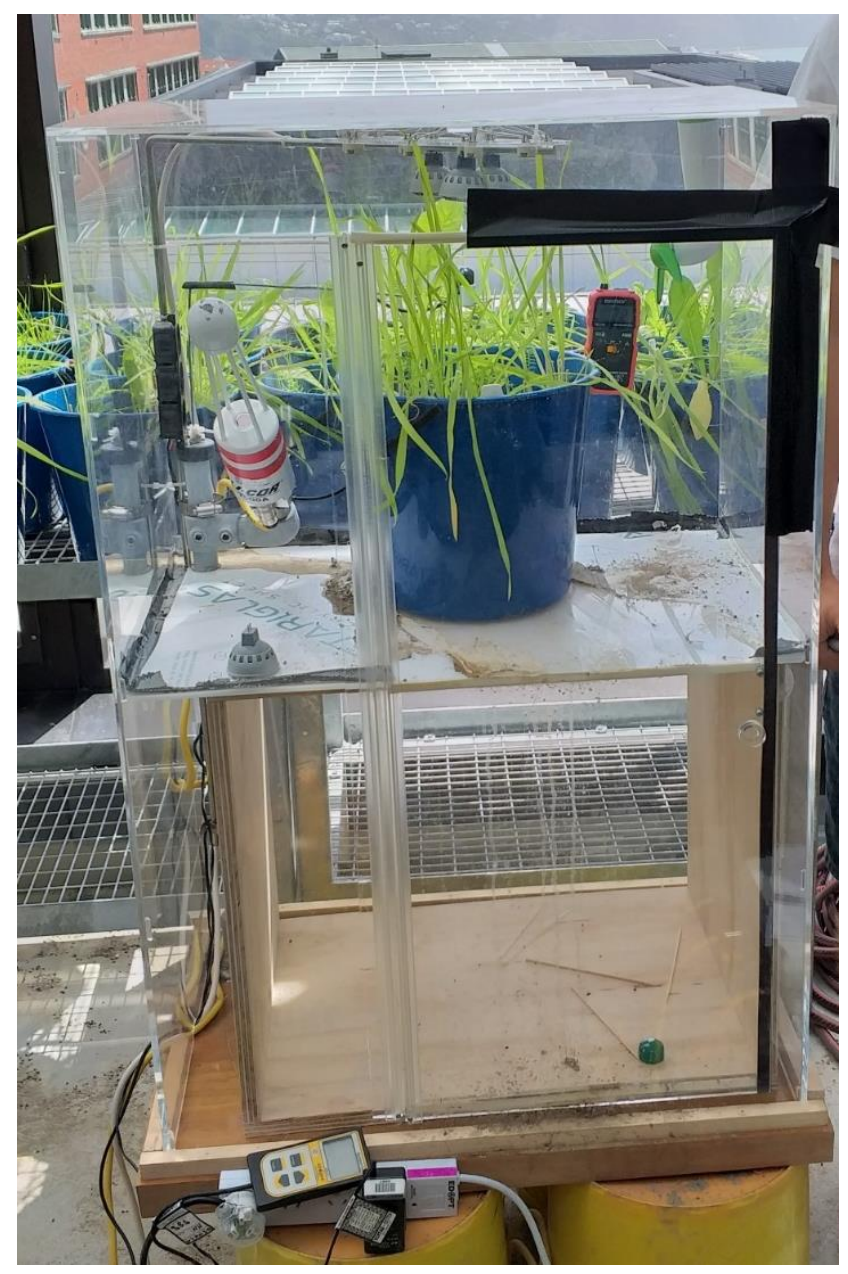

Figure 2. 3: Plastic NEE chamber with a Li-COR 7500A infrared gas analyser attached inside

\subsubsection{Above and below biomass method}

Once the experiment had been grown for 50 days from the addition of the late arrival plant, harvesting of both above ground and below ground biomass took place. Above ground biomass for each individual plant was collected at harvest by clipping individuals from their roots at the root collar and placing the above ground portions of the plant into labelled paper bags. These were then placed into drying ovens set to $60^{\circ} \mathrm{C}$. After $\sim 1$-week drying time the dry above ground biomass weights were measure for each individual.

In the species richness treatment with only 3 plants/pot, below ground plant biomass was collected for each individual. For the species richness treatments with either 5 or 9 plants per pot, belowground biomass of all species was pooled by pot. Bulk soil was carefully removed from root samples by shaking and rinsing in tap water. Root samples were then refrigerated 
for later processing. Once harvest was complete all the fresh biomass was rewashed thoroughly to particles caught in the roots. Each plant in all of the communities 1 were weighed as fresh weights then combined and subsampled for lipid extraction. The subsample for each treatment for all below ground biomass was weighed to $\sim \mathrm{g}$ of material however in cases where there wasn't enough material all the root biomass was used as the subsample for lipid extraction. Where there was excess biomass in community one, it was then pooled and put into the drying ovens for dry weights to be measured. For the rest of the communities 2 and 3 all below ground biomass was pooled subsampled for lipid extraction and then placed into a drying oven for dry weights to be taken. Dry weights for above and below biomass were taken on an analytical balance with $\pm 1 \mathrm{mg}$ precision.

Soil samples from each treatment pot were taken for lipid analyses. A subsample was taken from each treatment pot after giving the soil a mix to allow for good randomisation of the subsample. Later a subsample of the soil subsample was taken for lipid analyses.

\subsubsection{Neutral Lipid extraction method}

The AMF biomarker 16:1w5 was extracted from both the pooled roots and pooled soil samples from each treatment pot for all the unsterilised soil treatment and a subsample of both the inoculated and sterilised soil treatments. The extraction method used was a modified version of Bligh and Dyer (1959) and Buyer and Sasser (2012). A few modifications to this original method have been included below:

Roots and soil samples were lyophilised in a freeze drier at room temperature for at least 24 hours. Roots and soil were then weighed into glassware tubes ready for lipid extraction. For roots, between $0.1 \mathrm{~g}$ and $0.2 \mathrm{~g}$ of root was weighed into tubes. For soil between $1 \mathrm{~g}$ and $1.5 \mathrm{~g}$ was weighed into tubes for each extraction, exact masses of samples were recorded. $4 \mathrm{ml}$ of Bligh-Dyer extractant containing internal standard NL 19:0 was added to each individual sample. All tubes were then sonicated $10 \mathrm{~min}$ at room temperature before being rocked for $2 \mathrm{hrs}$ in the dark. After centrifuging for $10 \mathrm{~min}$ the liquid phase was transferred to clean glassware tubes with a prepared mixture of $1.0 \mathrm{ml}$ each of chloroform and water. Tubes were vortexed $5 \mathrm{~s}$ and centrifuged $10 \mathrm{~min}$. The lower phase containing the extracted lipids were removed using a glass pipette and transferred into new clean glass tubes, which was then evaporated using nitrogen at room temperature. Samples were stored overnight at $-20{ }^{\circ} \mathrm{C}$. 
Neutral lipids were the only class of lipids being extracted using this method and these were separated by solid phase extraction (SPE) using a 96-well SPE plate containing $50 \mathrm{mg}$ of silica per well. $3 \times 1 \mathrm{ml}$ of both methanol and then chloroform was used to condition the wells being used for separation. The samples being stored overnight were dissolved in $1 \mathrm{ml}$ chloroform, transferred to the SPE plate, and allowed to pass through the silica. Each well was then washed with $1 \mathrm{ml}$ of chloroform and the whole sample collected at this stage into a glass Durham tube due to the elution of the neutral lipid fraction. The sample collected was then evaporated under nitrogen at room temperature.

The samples were analysed using gas chromatography and mass spectrometry. The GC used was a Shimadzu GCMS-QP2010 Plus equipped with an autosampler and a split-splitless inlet was used. Both a split ratio of 10:1 and 30:1 were used with Helium carrier ga (ultra high purity) at $1.06 \mathrm{ml} / \mathrm{min}(39.7 \mathrm{~cm} / \mathrm{sec})$ through a Restek RXi5-Sil $(29 \mathrm{~m} \mathrm{x} 0.25 \mathrm{~mm} \mathrm{ID} \mathrm{x}$ $0.25 \mu \mathrm{m}$ film thickness) column. The sample was injected at $260^{\circ} \mathrm{C}$ with a gradient : $140^{\circ} \mathrm{C}$ start, $2 \mathrm{~min}$ hold and $3^{\circ} \mathrm{C} / \mathrm{min}$ to $240^{\circ} \mathrm{C}, 3 \mathrm{~min}$ hold. The mass spectrometer used was a Shimadzu GCMS-QP2010 Plus. It has a 2.5 min solvent cut time with a $200^{\circ} \mathrm{C}$ ion source temperature and a $260^{\circ} \mathrm{C}$ Interface temperature. And a detector Voltage relative to the tuning result $(0-1000 \mathrm{~V})$.

\subsection{Statistical analyses}

Evaluation of data normality and homogeneity of variance as well as statistical analyses were carried out on data using the integrated RStudio tool ( $\mathrm{R}$ version 3.6.1; R Core Team, 2019). Data distributions were evaluated with exploratory histograms and if assumptions of normality and homogeneity of variance were violated, data was transformed (log and square root) and distribution rechecked to see if assumptions were better met. Analysis using raw data was then compared to that from transformed data and no significant differences were found, thus raw data was used for analysis for all response variables to keep analysis between variables consistent. The package lme4 (Bates, Mächler, Bolker, \& Walker, 2015) was used to create mixed models followed by lmertest using the function "step" to create a backward reduced fixed-effect table and a backward reduced random-effect table. Where the "step" function wasn't appropriate both "ranova" and "drop1" functions were used. Using this method of model selection, best fit models for each response variable were selected. When the "step" function was used the "emmeans" function was used to look at pairwise relationships of fixed factors. Where mixed factor models were not required, a linear model was used to find the best fit model using the "heplots" package and analysis of variance (ANOVA) function. For both mixed effects and linear models, significant results were 
reported as $\mathrm{p}<0.05$ and interaction effects were only retained in the model significant $(\mathrm{p}<0.05)$. If a significant difference was found between factors using ANOVA, Tukey posthoc analyses were performed.

\subsubsection{Productivity response to richness and interaction trait}

NEE

A linear mixed model was used to analyse the Net Ecosystem Exchange data. A best-fit model was found using the step function and a backward reduced fixed-effect table and a backward reduced random-effect table. NEE was best represented as a response of community type and the random nested factor of the plant trait of the middle plant species and the random factor of NEE runs.

\section{Biomass}

\section{Total, above and below ground biomass}

A linear mixed model was used to analyse the total plant biomass, above, and below ground. A best fit model was found using the "ranova" function with an ANOVA-like table for random-effects: Single term deletions and the "drop1" function Single term deletions using Satterthwaite's method. A linear model was found to best fit the total, above and below ground biomass data with community type as a fixed factor.

\section{Individual biomass}

A linear mixed model was used to analyse the individual plant biomass. A best fit model was found using the "ranova" function with an ANOVA-like table for random-effects: Single term deletions and the "drop1" function Single term deletions using Satterthwaite's method. There was no significant effect of random factors, therefore a ANOVA model was undertaken. A linear model was found to best fit the individual plant biomass with community type, late arrival plant trait, and arrival status of individual plants as fixed factors.

\section{NLFA}

\section{Total Soil NLFA}

A linear mixed model was used to analyse the total soil NLFA. A best-fit model was found using the step function and a backward reduced fixed-effect table and a backward reduced 
random-effect table. There were no significant effects of both random factors and fixed factors.

\section{Per gram roots $N L F A$}

A linear mixed model was used to analyse the total root NLFA. A best fit model was found using the "ranova" function with an ANOVA-like table for random-effects: Single term deletions and the "drop1" function Single term deletions using Satterthwaite's method. There were no significant effects of random factors, therefore an ANOVA model was used. Total root NLFA data was described best by the community type as a fixed factor. The same model outcome was found when analysing the NLFA total root data.

\subsubsection{Environmental Context}

NEE

A linear mixed model was used to analyse the Net Ecosystem Exchange data. A best fit model was found using the "ranova" function with an ANOVA-like table for random-effects: Single term deletions and the "drop1" function Single term deletions using Satterthwaite's method. The best fit model found NEE as a response of community type, soil type, and the random factor of NEE runs.

\section{Biomass}

\section{Total, above and below ground biomass}

A linear model was used to analyse the total plant biomass. A linear model was found to best fit the total biomass data with community type, soil type, and late arrival plant trait as fixed factors.

Both above and below ground biomass had linear best models using the ANOVA function where the biomass was best described using community type, soil type, and late arrival plant trait as fixed factors.

\section{Individual biomass}

A linear mixed model was used to analyse the individual plant biomass. A best-fit model was found using the step function and a backward reduced fixed-effect table and a backward reduced random-effect table. A mixed linear model was found to best fit the total root data 
with community type, late arrival plant trait, soil type, arrival status of individual plants as fixed factors and community pot number as the random factor.

\section{NLFA}

\section{Total Soil NLFA}

A linear mixed model was used to analyse the total soil NLFA. A best fit model was found using the "ranova" function with an ANOVA-like table for random-effects: Single term deletions and the "drop1" function Single term deletions using Satterthwaite's method. There were no significant effects of random factors, therefore an ANOVA model was used. There were no significant effects of both random factors and fixed factors.

\section{Total roots NLFA}

A linear mixed model was used to analyse the total root NLFA. A best fit model was found using the "ranova" function with an ANOVA-like table for random-effects: Single term deletions and the "drop1" function Single term deletions using Satterthwaite's method. There were no significant effects of random factors, therefore an ANOVA model was used. Per gram root NLFA data was described best by the fixed factor community type and soil type. 


\section{Chapter 3 - Results}




\section{Results}

\subsection{Productivity response to plant richness and interaction trait}

\subsubsection{NEE}

When photosynthesis exceeds respiration the slope of carbon flux, NEE, is negative. A more negative NEE indicates a greater photosynthesis by the plant community. At the beginning of the productivity response to richness and plant interaction trait experiment (Run 1, one week after the addition of a specialist or generalist plant species into the middle of the community), there were no observed significant differences in NEE among plant interaction trait treatments (control, generalist or specialists) in community 1 (Figure 3.1). Community 2 had significantly more negative NEE with an interaction specialist relative to the other communities (Tukey post-hoc, p<0.05; Figure 3.1). In contrast, in community 3, which had the highest species richness, the addition of a specialist led to a significantly more positive NEE relative to other treatments, indicating less photosynthesis or more respiration in comparison to the control or addition of generalist (Tukey post hoc, $\mathrm{p}<0.0001$; Figure 3.1). However, when Run 2 was measured (eight weeks after the addition of a specialist or generalist plant species into the middle of the community), the addition of a specialist plant to community 3 resulted in a significantly more negative slope of carbon flux (Tukey post-hoc, $p$ $<0.005$, Figure 3.1). In Run 2, specialists were the only plant category to show a significant difference across communities $\left(F_{2,42}=26.615\right.$, $p<0.001$; Figure 3.1). Specialists had a much greater effect on the drawdown of carbon in community 3 leading to much more negative NEE when they were added to community 3, than in community 1 and 2 (Tukey post-hoc, $\mathrm{p}<0.001$; Figure 3.1).This result is in line with my hypothesis that specialists may support greater productivity because they reduce competition for interacting (i.e. niche-partitioning). However, after 11 weeks (Run 3), this trend disappeared and while significant difference among treatments existed, they were idiosyncratic and none conformed to my hypotheses (Tukey post-hoc, $p<0.05$, Figure 3.1). The biggest change across Run 3 was seen in community 3 , where NEE in the control treatment was now significantly lower than the NEE with the addition of either a generalist or specialist (Figure 3.1). 

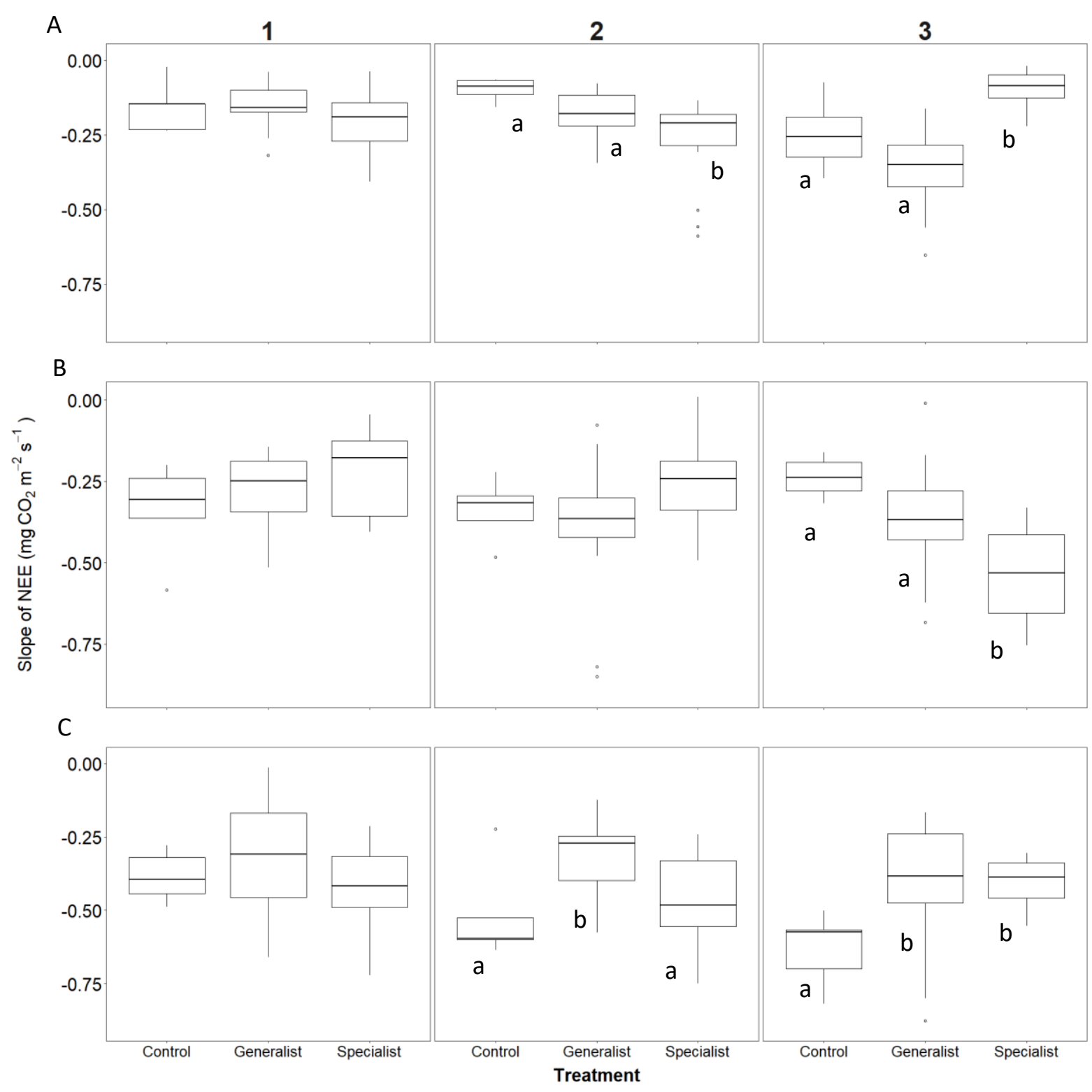

Figure 3. 1: Slope of Net Ecosystem exchange in $\mathrm{mg}$ of carbon dioxide in meters per second. Treatment along the bottom shows the interaction trait of the late arrival plant. Runs are represented by A, B and C, where A is Run 1, B is Run 2, and C is Run 3. The columns represent the different community types with the left-hand column as community 1 , the middle column as community 2 and the right-hand column as community 3 . Significant differences between interaction traits (Tukey post hoc test, $\mathrm{p}<0.05$ ) are indicated by different lowercase letters above the boxplots 


\subsubsection{Plant biomass - Community Scale}

Plant interactions traits did not significantly affect total plant biomass in the productivity response to plant richness and interaction trait experiment. In general, late arriving plants were small at the time of harvest and therefore specialists and generalists had similar, and little, impact on total community biomass.

Total plant biomass, and the allocation of plant biomass above and below ground differed significantly among community types. Total plant biomass was significantly greater in community 3 with 9 plant species (mean \pm standard error $=2.81 \pm 0.13$ ) than in either community $1(1.89 \pm 0.16)$ and $2(2.04 \pm 0.09)$, with no differences seen between the latter two communities. The same patterns of significance were observed when only above ground biomass was analysed. However, belowground biomass was significantly greater in the pots with 5 or 9 species (community $2(0.84 \pm 0.06)$ and $3(0.97 \pm 0.05)$ ), than in community 1 $(0.58 \pm 0.06)$ which had only 3 plants $\left(F_{2,101}=12.533, p<0.001\right.$, Figure 3.2). 


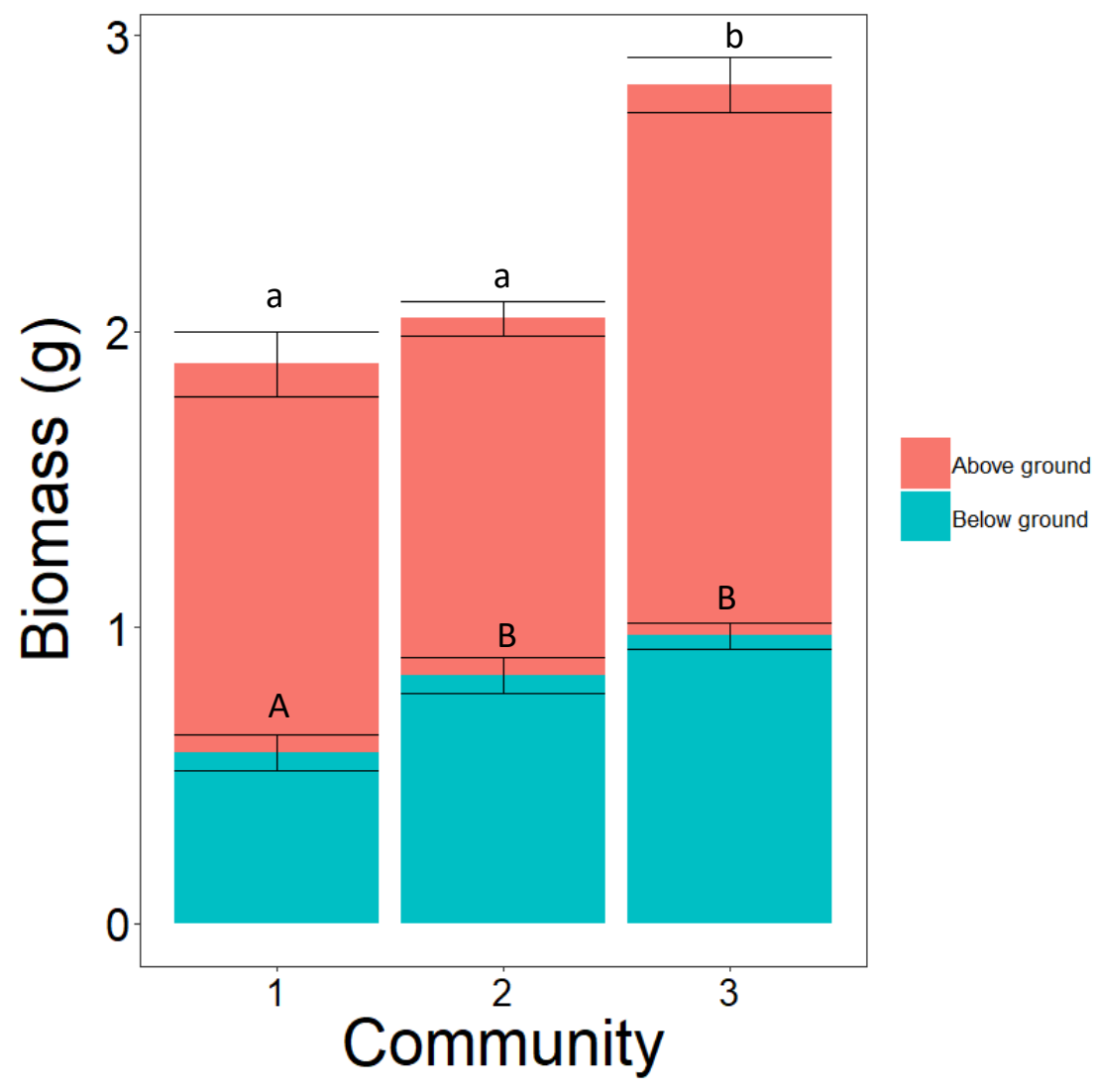

Figure 3. 2: Total plant biomass (g) of community types 1 (3 plants), 2 (5 plants) and 3 (9 plants), separated into above ground biomass (orange) and below ground biomass (blue). Bars show means \pm SE $(n=5)$. Capital letters show the significant differences $(p<0.05$; Tukey post-hoc) in the below ground biomass and the lower-case letters show significant differences in both above ground biomass and total biomass.

\subsubsection{AMF biomass}

The concentration of NLFA biomarker for AMF was measured in a subsample of soil from each pot, as well as in a random subsample of pooled roots from the plant community in each pot. The mean concentration of $16: 1 w 5$ in soil was $12.14 \mu \mathrm{mol}$ per Total soil per treatment pots and did not differ by treatments (species richness and plant interaction trait). Figure 3.3 shows the concentration of the NLFA biomarker for AMF in soils in the three communities (3, 5 or 9 species). 


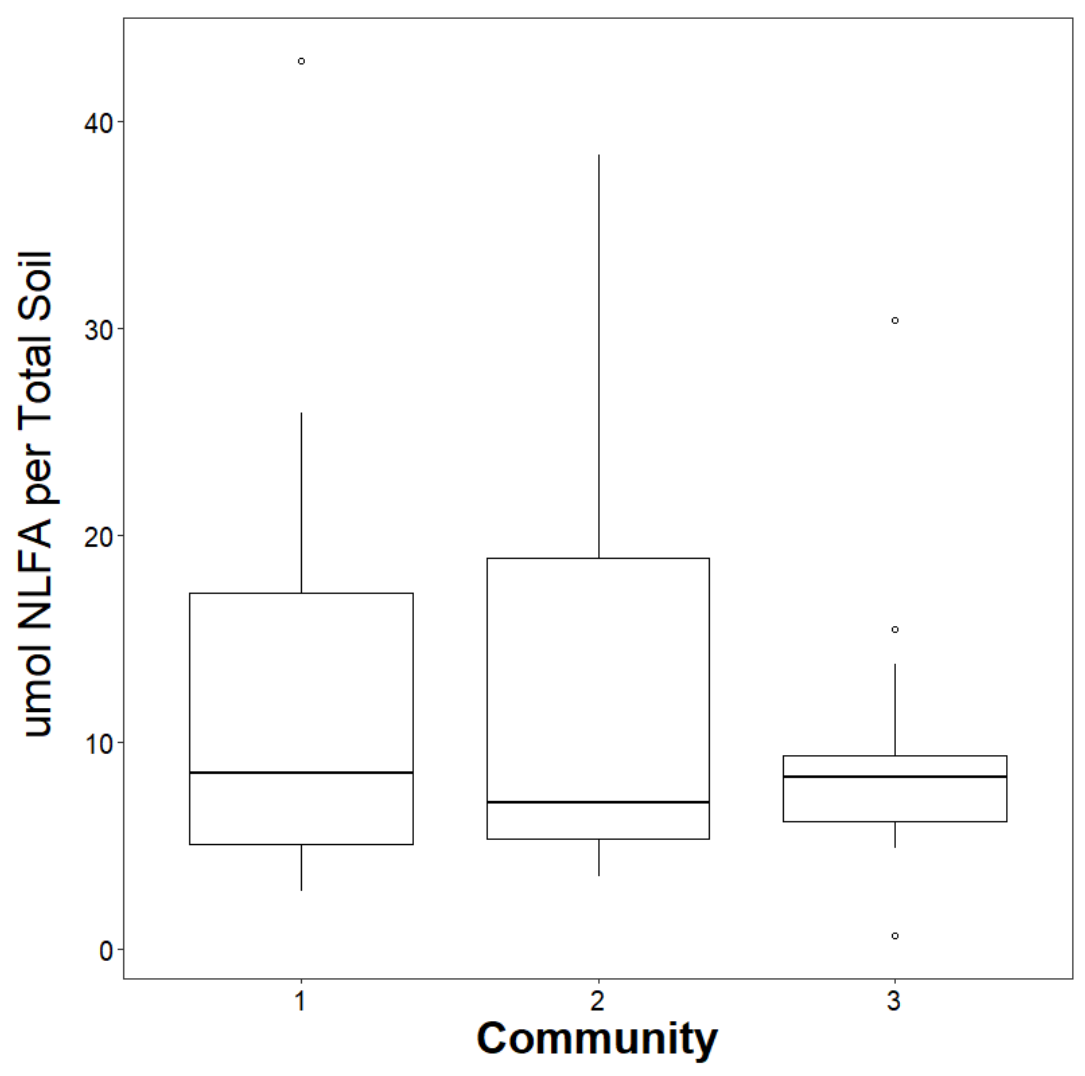

Figure 3. 3: The concentration of $16: 1 w 5$, the NLFA biomarker for AMF, in $9.5 \mathrm{~kg}$ of growth medium (individual treatment pot volume), $\mu$ mol per Total soil per treatment pots.

Plant species richness had significantly positive effects on the amount of the AMF biomarker 16:1w5 in plant roots; and this was true both on a per gram root basis and in total. For example, the total amount of the AMF biomarker 16:1w5 significantly greater in pooled roots when plant richness was greater (community type) $\left(\mathrm{F}_{1,96}=11.862\right.$, $\mathrm{p}<0.001$; Figure 3.4). Community 2 and 3 had a significantly more 16:1w5 than community 1 (Tukey post-hoc; $\mathrm{p}<0.005$; Figure 3.4). However, the amount of 16:1w5 did not differ between communities 2 and 3 (Tukey post-hoc, $\mathrm{p}>0.05$; Figure 3.4). Both total 16:1w5 per root system and 16:1w5 per gram of roots followed this pattern in significance. The difference in the per gram per root model was slightly more significant due to the total root model having more variation in the total root biomass per treatment (Figure 3.4). 
1

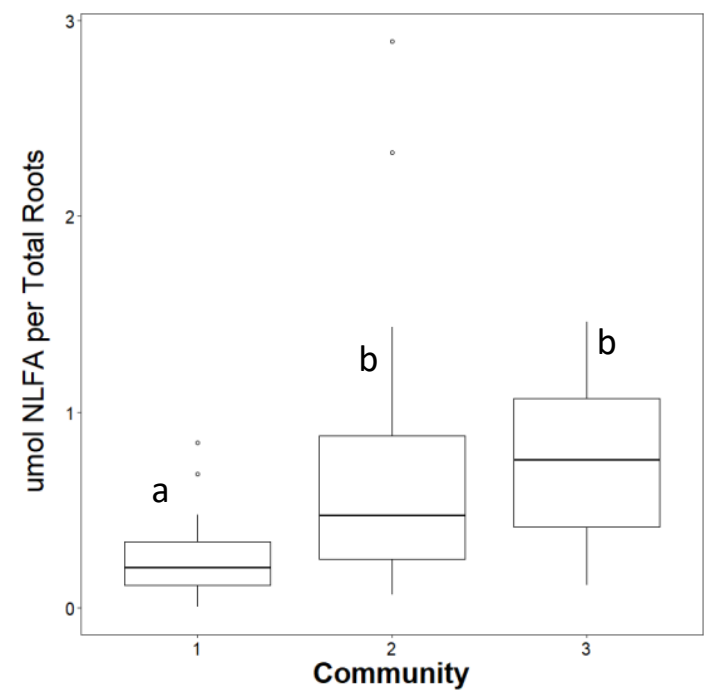

2

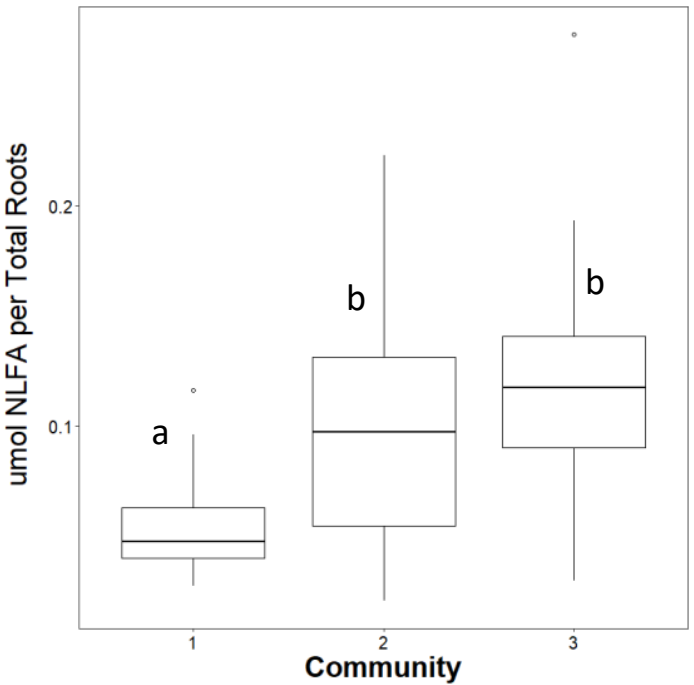

Figure 3. 4: 1 . The total amount of the NLFA biomarker for AMF 16:1w5 (in $\mu \mathrm{mol}$ ) per community root system per species richness treatment (3, 5 or 9 plant species). Significant differences between community types (Tukey pot-hoc, $\mathrm{p}<005$ ) are shown by lower case letters. 2. The total amount of the NLFA biomarker for AMF 16:1w5 (in $\mu \mathrm{mol}$ ) per gram of root per species richness treatment (3, 5 or 9 plant species). Significant differences between community types (Tukey pot-hoc, $\mathrm{p}<005$ ) are shown by lower case letters

\subsubsection{Plant biomass - Individual Scale}

Variation in individual biomass of plants in the initial communities (i.e. prior to addition of the late arrivals) revealed that species richness had significant effects on individual plant biomass $\left(\mathrm{F}_{2,566}=24.505, \mathrm{p}<0.001\right.$; Figure 3.5$)$. There was large variation plant biomass both among species and also within species across the different community richness's. For example, in community 1 Holcus lanatus was significantly bigger than in communities 2 and 3 (Tukey post-hoc, p<0.001; Figure 3.5). Mean biomass of Holcus lanatus in community 1, which contained only 3 plant species, was $0.86 \mathrm{~g}$ compared to only $0.38 \mathrm{~g}$ and $0.53 \mathrm{~g}$ in community 2 and 3, respectively. The mean biomass of Achillea millefolium in community 1 was also significantly bigger than both its respective individuals in both community 2 and 3 (Tukey post-hoc, $\mathrm{p}<0.001$; Figure 3.5 ). Cichorium intybus was significantly bigger in community 2 than community 3 (Tukey post-hoc, $p<0.05$ ), showing that the plant species that were grown in communities with few plants tended to have grown bigger than in communities with more plants (Figure 3.5). 


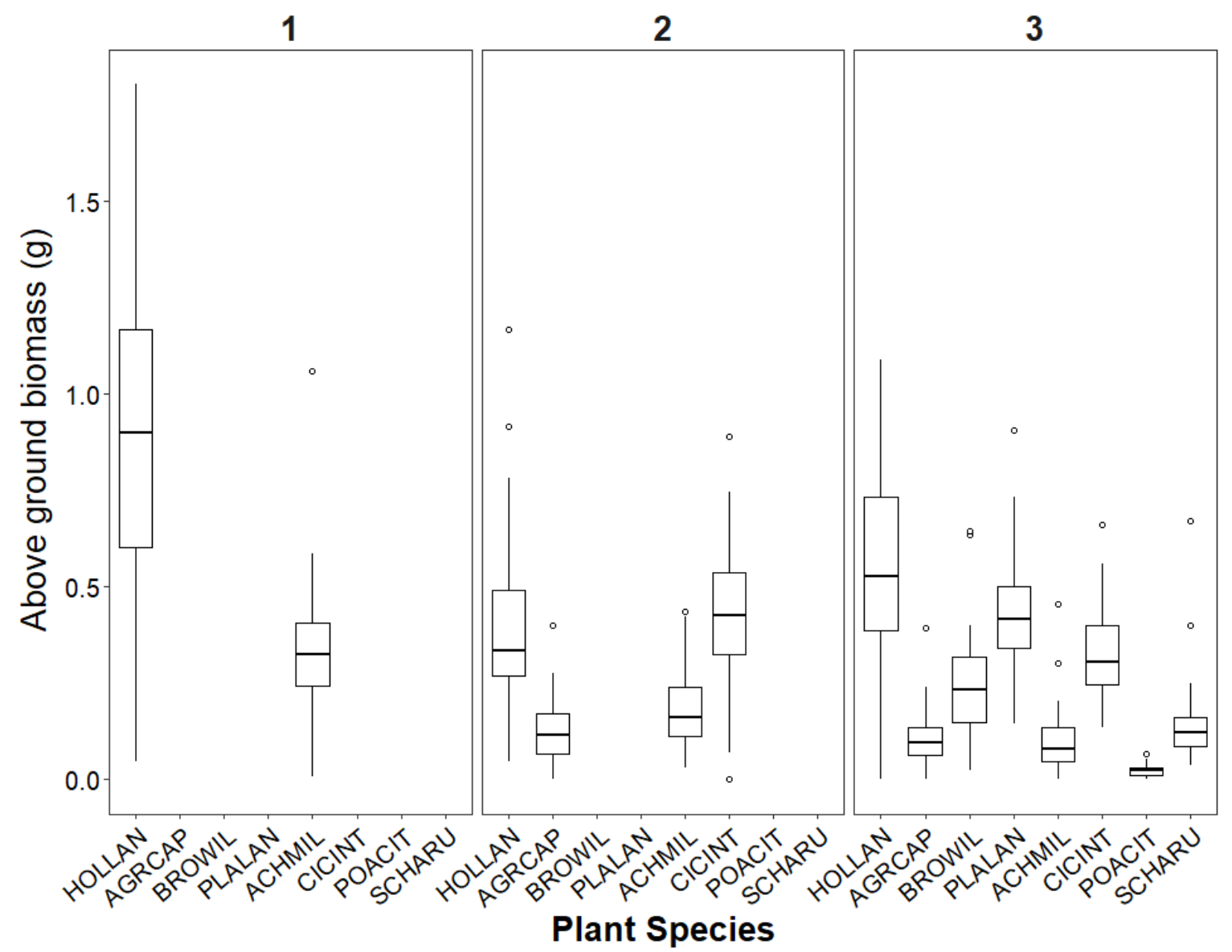

Figure 3. 5: Individual above ground biomass ( $\mathrm{g}$ ) for each plant species in this experiment for first arrivals. Plant species are shown here using the six-letter code. Letters 1, 2, and 3 represent the respective community treatments.

Individual "late arrivals" had very low biomass overall. Despite this, the results showed significant species and richness effects on the biomass of late arrivals. In general, plants were larger when grown at lower densities, which led to a significant effect of community type on the biomass of later arrivals $\left(\mathrm{F}_{2,86}=6.387, \mathrm{p}<0.005\right.$; Figure 3.6). Across all communities, Bromus willdenowii grew larger than other plant species $\left(\mathrm{F}_{5,83}=20.227, \mathrm{p}<0.001\right.$; Figure 3.6). Within individual plant species, plant biomass depended on the richness of the community. For example, Holcus lanatus had significantly greater biomass when grown in community 1 than when grown in communities 2 and 3 (Tukey post-hoc, $p<0.05$; Figure 3.6). Bromus willdenowii when grown in community 2 had significantly greater biomass than when grown in community 3 (Tukey post-hoc, p<0.05; Figure 3.6) as was the same with Cichorium intybus (Tukey post-hoc, $\mathrm{p}<0.05$; Figure). The decrease in individual plant biomass is an 
interesting result as the total biomass of the overall communities increased as plant species in communities increase $(3,5$, and 9 plants).

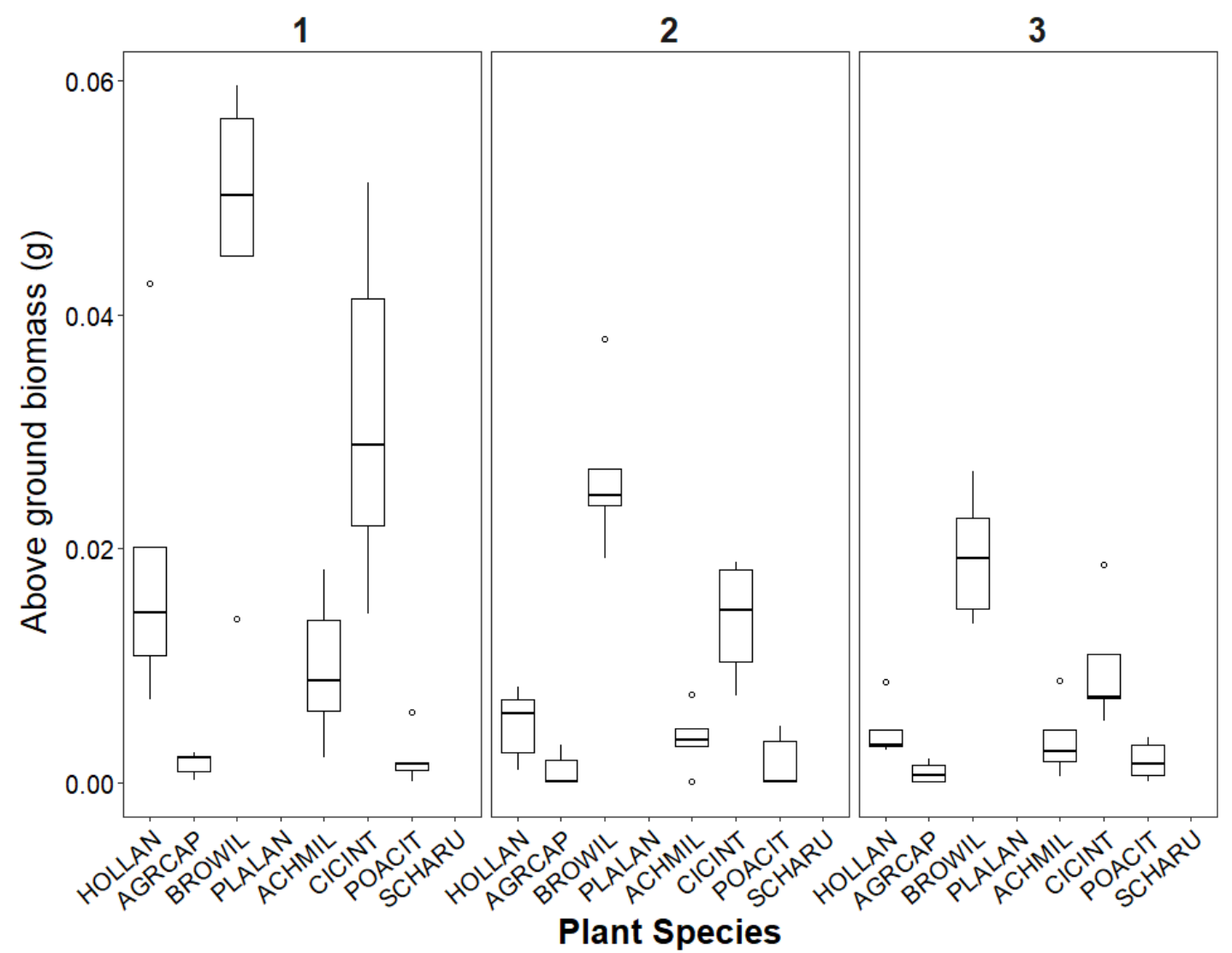

Figure 3. 6: Individual above ground biomass ( $\mathrm{g}$ ) for each plant species in this experiment for "late arrival" plants planted into the original communities. Plant species are shown here using the six-letter code. Letters 1, 2, and 3 represent the respective community treatments.

\subsection{Environmental Context Experiment}

\subsubsection{NEE}

The environmental context experiment tested the effect of species richness, plant interactions traits and varying conditions of the soil microbial community on community productivity. In general, more production (more negative NEE) under sterile and reinoculated soils than in normal soils, which was contrary to expectations. Despite that the environmental context 
experiment included the addition of only the strongest generalist and specialist plant species $(\mathrm{G} 1$ = Holcus lanatus, $\mathrm{S} 1=$ Achillea millefolium $)$ where there was no significant effect of plant interaction trait on NEE under any soil condition or community type.

However, I observed significant effects of soil types and species richness over time. When NEE was measured across a range of soil types (normal, sterile and reinoculated), there were differences in NEE. In Run 1, plant communities grown in sterile soils had significant differences in NEE than when grown in normal soil, while those that had been reinoculated had significantly different NEE values to communities grown in sterile soils $\left(F_{2,132}=16\right.$, p $<0.001$; Figure 3.7). In Run 2, plant communities grown in normal soil were significantly different to communities grown in both inoculated and sterilised soils $\left(\mathrm{F}_{2,132}=49.145\right.$, $\mathrm{p}<0.001$; Figure 3.7). In Run 3, plant communities grown in sterile soils had significant differences in NEE than when grown in normal soil, while those that had been reinoculated had significantly different NEE values to communities grown in sterile soils as well $\left(\mathrm{F}_{2,132}=\right.$ 9.81, $\mathrm{p}<0.001$; Figure 3.7).

At the beginning of the experiment, (Run 1) soil type significantly affected carbon draw down with greater NEE in sterile soils however with each soil type there were no differences between plant communities. In Run 2 there was no significance within communities in both normal and sterile soils. However, in inoculated soil community 2 had a more negative NEE slope than community 1 , where community 1 and 3 weren't significantly different (Tukey post-hoc, $\mathrm{p}<0.05$; Figure 3.7). In Run 3 of NEE measurements showed the most differences in the affect's community type draw down of carbon. In normal soil, community 2 and 3 had a more negative NEE than community 1 (Tukey post-hoc, $\mathrm{p}<0.05$; Figure7). In inoculated soil, community 1 supported significantly more negative NEE than both community 2 and 3 (Tukey post-hoc, $\mathrm{p}<0.05$; Figure 3.7).

While plant species richness affected NEE, patterns were not consistent over time or among soil conditions. Across soil types in Run 1, community 1 in both sterile and inoculated soils had a significantly more negative slope of carbon flux than when grown in normal soil (Tukey post-hoc, $\mathrm{p}<0.05$; Figure 3.7 ). Community 3 had a more negative slope of carbon flux in sterile soil than both normal and inoculated soils (Tukey post-hoc, p<0.005; Figure 3.7). Across soils in Run 2, community 1 grown in sterile and inoculated soil had a significantly more negative slope of carbon flux than when grown in normal soil (Tukey post-hoc, $\mathrm{p}<0.005$; Figure 3.7). Much the same as community 1 in Run 2, community 2 also had a significantly more negative slope of carbon flux in sterile and inoculated soil than when grown in normal soil (Tukey post-hoc, $\mathrm{p}<0.001$; Figure 3.7). Community 3 showed exactly the same results as community 1 and 2 in Run 2 when comparing carbon drawn down across 
soil types (Tukey post-hoc, $\mathrm{p}<0.001$; Figure 3.7 ). Across soils in Run 3, community 1 had a significantly more negative slope of carbon flux when grown in sterile and inoculated soil than when grown in normal soil (Tukey post-hoc, $\mathrm{p}<0.001$; Figure 3.7). Community 3 had a more negative slope of carbon flux when grown in sterile soil than when grown in both normal and inoculated soil (Tukey post-hoc, p<0.005; Figure 3.7).

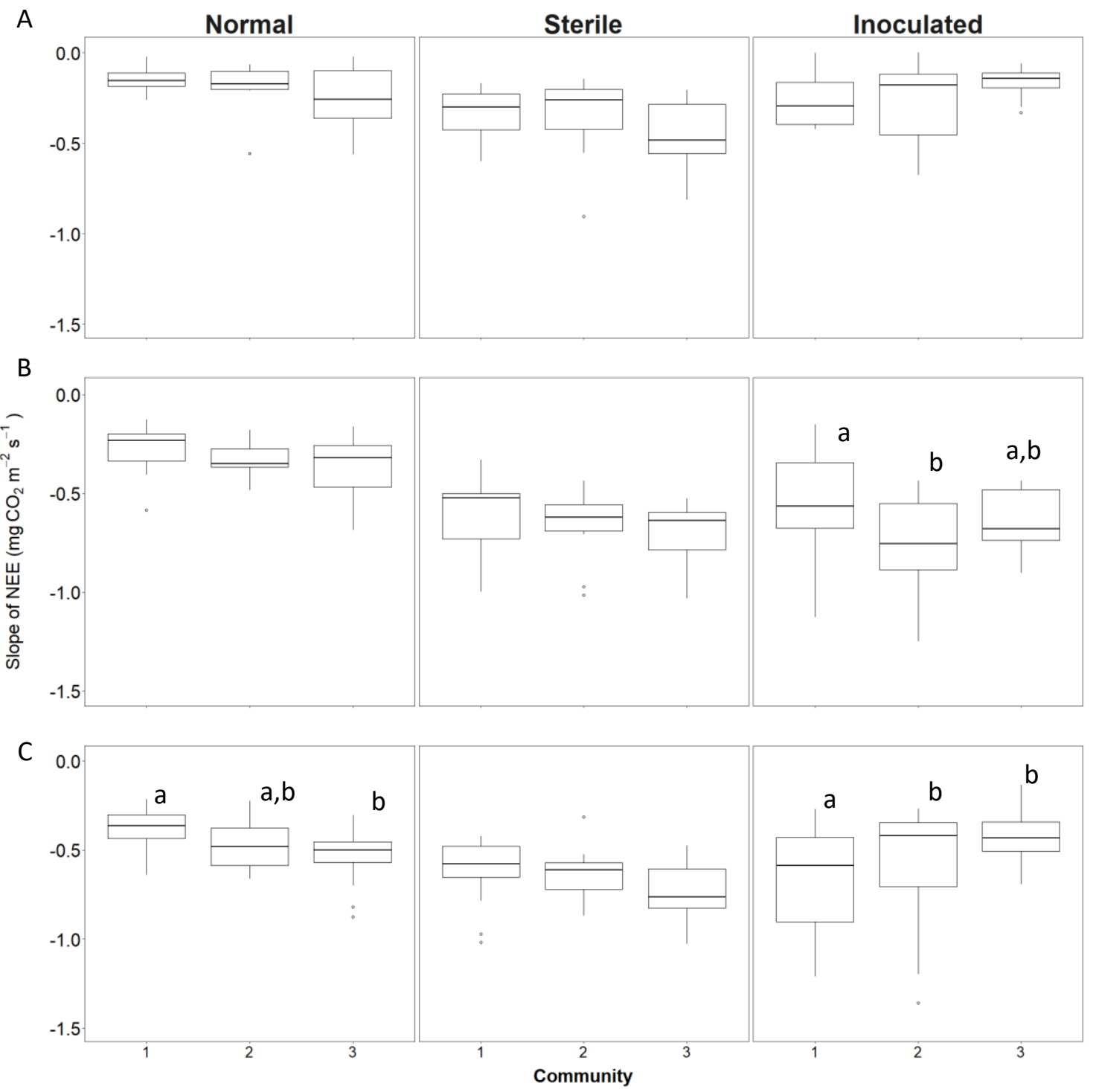

Figure 3. 7: Slope of Net Ecosystem exchange (carbon draw down) in mg of carbon dioxide in meters per second. Treatment along the bottom shows the respective community being measured. Runs are represented by A, B and C, where A is Run 1, B is Run 2, and C is Run 3. The columns represent the different soil types. 


\subsubsection{Plant biomass - Community Scale}

As with the NEE results, total, above and below-ground community-level biomass indicated that plant production was significantly greater in sterile and inoculated soils than in normal soil, which was contrary to our expectations (Tukey post-hoc, p<0.001; Figure 3.8).nTotal community biomass was nearly 3 times as large in the sterile and reinoculated soils than in normal soil.

Within soil types, total community biomass differed among communities only in the sterile soil treatment $\left(\mathrm{F}_{2,42}=5.3575, \mathrm{p}<0.05\right)$. However, this did not align with differences in plant species richness. In sterile soil, communities $1(5.35 \pm 0.65)$ and $3(6.30 \pm 0.34)$ had significantly higher biomass than community 2 ( $4.20 \pm 0.29$; Tukey post-hoc, $\mathrm{p}<0.05$; Figure 3.8), despite its intermediate species richness.

Above ground biomass followed a very similar pattern to the total biomass results. In the normal soil, community $3(1.687720 \pm 0.13774068)$ had greater biomass than both community $2(1.127960 \pm 0.07495473)$ and $1(1.381922 \pm 0.19619695)$ (Tukey post-hoc, $\mathrm{p}<0.05$; Figure 3.8). The same pattern was observed in sterile soil; community 3 (3.253367 \pm 0.16739500 ) had the greatest biomass among communities (Tukey post-hoc; $p<0.05$; Figure 3.8). There were no significant differences in aboveground biomass between communities in inoculated soil. Above ground biomass increased in communities grown in both sterile and inoculated soils. The greatest above ground biomass was grown in inoculated soils. Inoculated soil had a significantly greater biomass than normal soil (Tukey post-hoc, $\mathrm{p}<0.001$ ); Figure 3.8) and sterile soil (Tukey post-hoc, $\mathrm{p}<0.05$; Figure 3.8).

Below ground biomass showed a slightly different pattern than total biomass and above ground biomass. In normal and inoculated soils, communities showed no significant differences. In the sterile soil treatment community $1(2.32 \pm 0.27)$ and $3(3.05 \pm 0.21)$ had the greatest biomass (Tukey post-hoc, $\mathrm{p}<0.05$; Figure 3.8 ). Community 1 had the greatest below ground biomass in sterile and inoculated soils (Tukey post-hoc, $\mathrm{p}<0.001$, Figure 3.8). Community 3 also had the greatest biomass in both sterile and inoculated soils (Tukey posthoc, $\mathrm{p}<0.001$; Figure 3.8). Community 2 increased in below ground biomass from normal soil $(0.95 \pm 0.07)$ through to inoculated soil $(2.84 \pm 0.32$; Tukey post-hoc, $\mathrm{p}<0.001$; Figure 3.8$)$. 


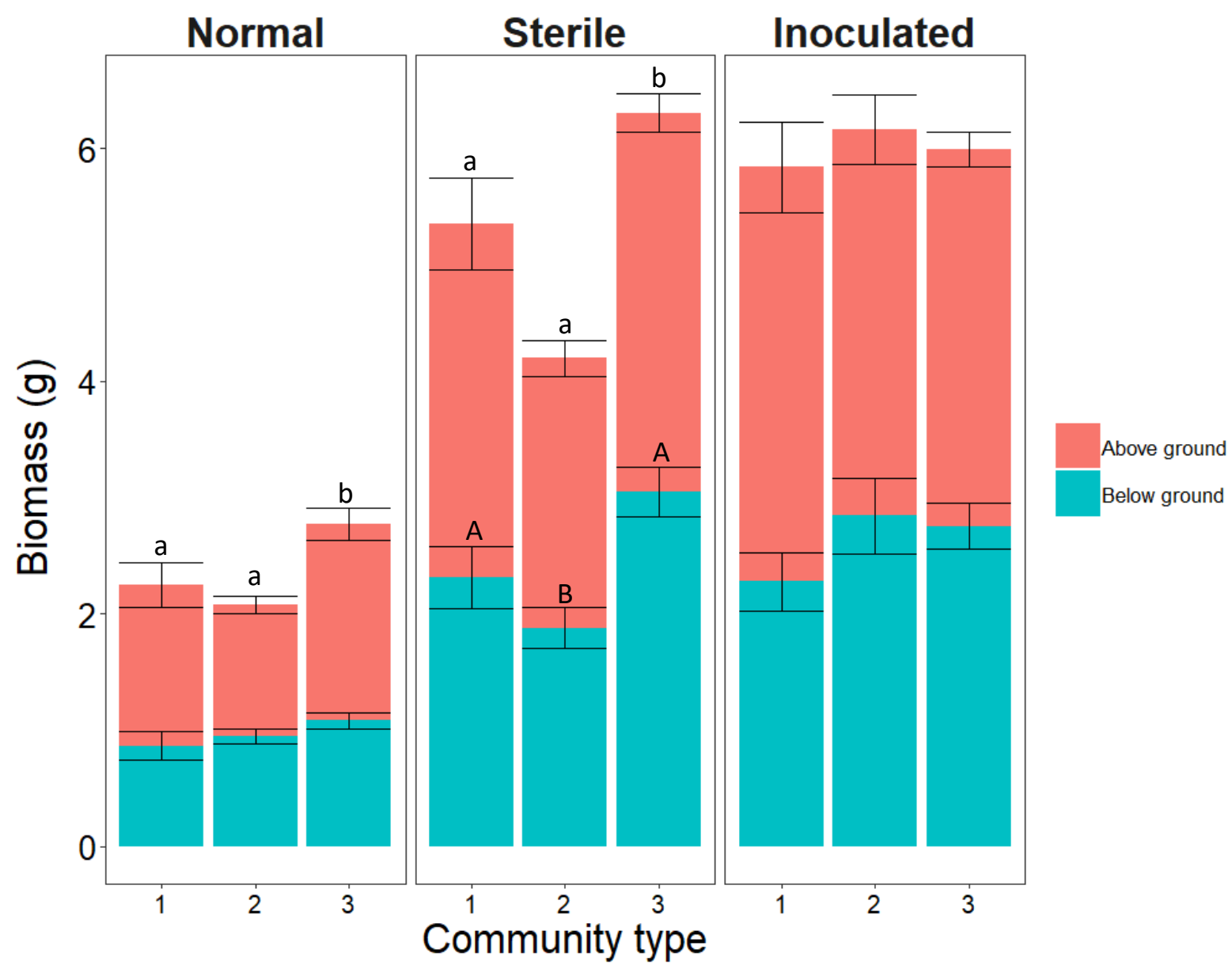

Figure 3. 8: Total community biomass in g. Above ground biomass in orange and below ground biomass in blue. Values presented are means with $\pm \operatorname{SE}(n=5)$. Panels represents three soil conditions; normal, sterilized, and sterilized but reinoculated soil. Capital letters show the significant differences $(\mathrm{p}<0.05$; Tukey post-hoc) in the below ground biomass and the lowercase letters show significant differences in the above ground biomass.

\subsubsection{AMF Biomass}

AMF production in soils, as measured through quantification of the 16:1w5 neutral lipid biomarker for AMF, was unaffected by soil type in the environmental context experiment When measuring the concentration of 16:1w5 in soils, total soil 16:1w5 showed no significant difference between communities within the three different soil types $\left(\mathrm{F}_{2,132}=\right.$ 0.8338 , $>0.05$; Figure 3.9). Across soil types there were significant differences in the total concentration of $16: 1 w 5$ for specific community types $\left(\mathrm{F}_{2,132}=13.598=\mathrm{p}<0.001\right.$; Figure 3.9). Community 2 had a significantly larger concentration of 16:1w5 in inoculated soil than in normal soil (Tukey post-hoc, $\mathrm{p}<0.005$; Figure 3.9 ). Community 3 had a significantly lower concentration of 16:1w5 in sterile soil when compared to normal soil (Tukey post-hoc, 
$\mathrm{p}<0.05$; Figure 3.9) but then had a significantly larger concentration of 16:1w5 in inoculated soil when compared to sterile soil (Tukey post-hoc, p<0.005; Figure 3.9).

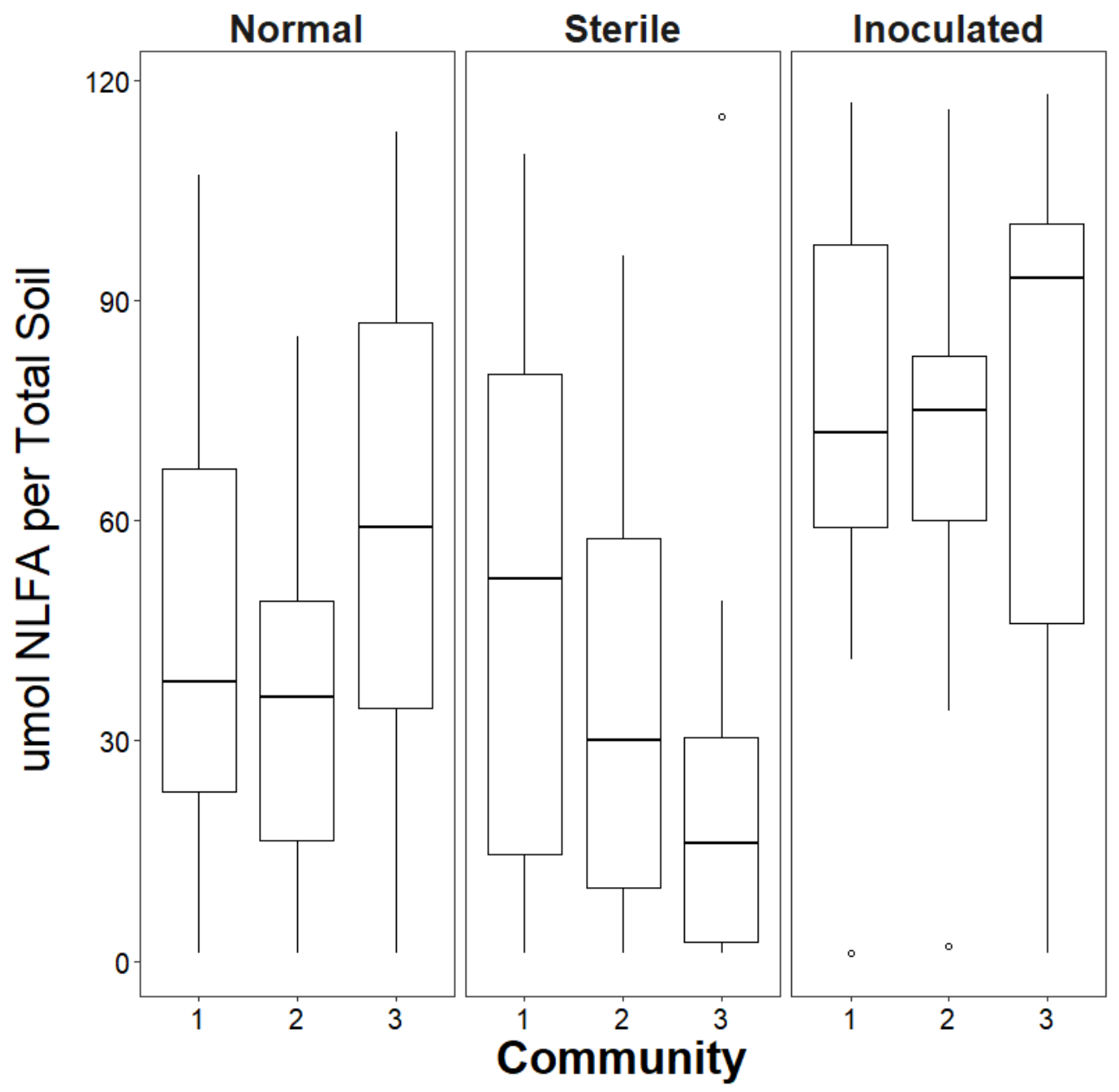

Figure 3. 9: Total NLFA biomarker for AMF 16:1w5 (in $\mu \mathrm{mol}$ ) per pot by species richness treatment (3, 5 or 9 plant species), in each of the three soil types; normal, inoculated and sterile. Significant differences between community types (Tukey pot-hoc, $\mathrm{p}<005$ ) are shown by lower case letters.

When considered on a per gram of root basis, plant species richness had significant effects on AMF biomass in plant roots. For example, in normal soil, community 2 (5 plants) and 3 (9 plants) had significantly greater amounts of the AMF biomarker than community 1 ( 3 plants; Tukey post-hoc, $\mathrm{p}<0.05$; Figure 3.10). However, no such effects of plant species richness on AMF biomass in plant roots occurred in sterile or inoculated soils. Rather, in sterile soil, community 1 supported significantly greater amounts of the AMF biomass in plant roots (Tukey post-hoc, $\mathrm{p}<0.05$; Figure 3.10 ) and the greatest concentration in inoculated soil 
(Tukey post-hoc, $\mathrm{p}<0.001$; Figure 3.10). The same pattern followed for both community 2 and 3 (Tukey post-hoc, $\mathrm{p}<0.05$; Figure 3.10). Between sterile and inoculated soil, there were no significant differences between each individual community.
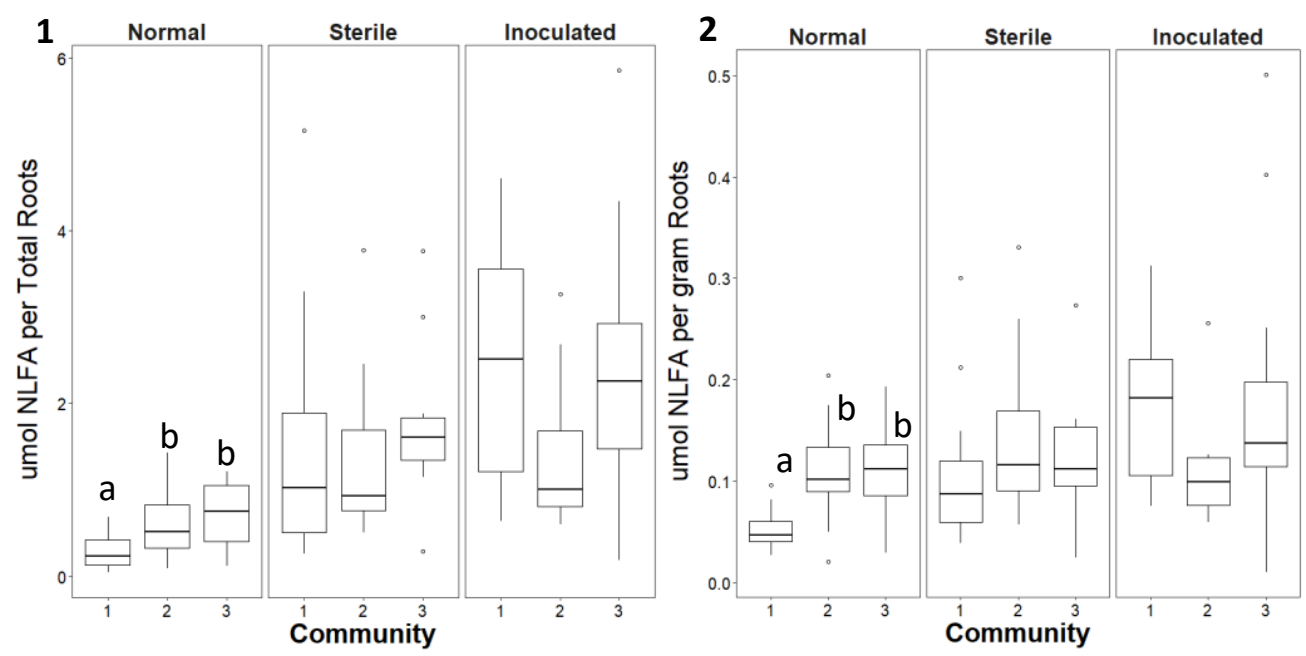

Figure 3. 10: 1 . The total amount of the NLFA biomarker for AMF 16:1w5 (in $\mu \mathrm{mol}$ ) per community root system per species richness treatment (3,5 or 9 plant species) in the three different soil types. Significant differences between community types (Tukey pot-hoc, $p<005$ ) are shown by lower case letters. 2. The total amount of the NLFA biomarker for AMF 16:1w5 (in $\mu$ mol) per gram of root per species richness treatment (3, 5 or 9 plant species) in the three different soil types.

\subsubsection{Plant biomass - Individual Scale}

The individual biomass of Holcus lanatus in the original community grown first in this experiment was significantly affected by community type $\left(\mathrm{F}_{2,125}=36.2034, \mathrm{P}<0.001\right.$; Figure $3.11)$, soil type $\left(F_{2,125}=37.2621, p<0.001\right.$; Figure 3.11), and the interaction between soil type and community type $\left(\mathrm{F}_{4,125}=4.9631, \mathrm{p}<0.001\right.$; Figure 3.11$)$. In community 1 , Holcus lanatus had significantly higher biomass than in both sterile and inoculated soil than in normal soil (Tukey post-hoc, $\mathrm{p}<0.001$; Figure 3.11). The biomass of Holcus lanatus did not significantly change in normal soil across communities. In sterile soil, Holcus lanatus had significantly greater biomass in community 1 than in community 2 and 3 (Tukey post-hoc, $p<0.001$; Figure 3.11). The same trend was true for Holcus lanatus in inoculated soil (Tukey post-hoc, $\mathrm{p}<0.001$; Figure 3.11). Of all individual plant species, only generalist Plantago lanceolate and specialist Achillea millefolium was not significantly affected by soil type. Across community 
types Achillea millefolium had significantly greater biomass in community 1 than in community 2 and 3 (Tukey post-hoc, p<0.005; Figure 3.11).

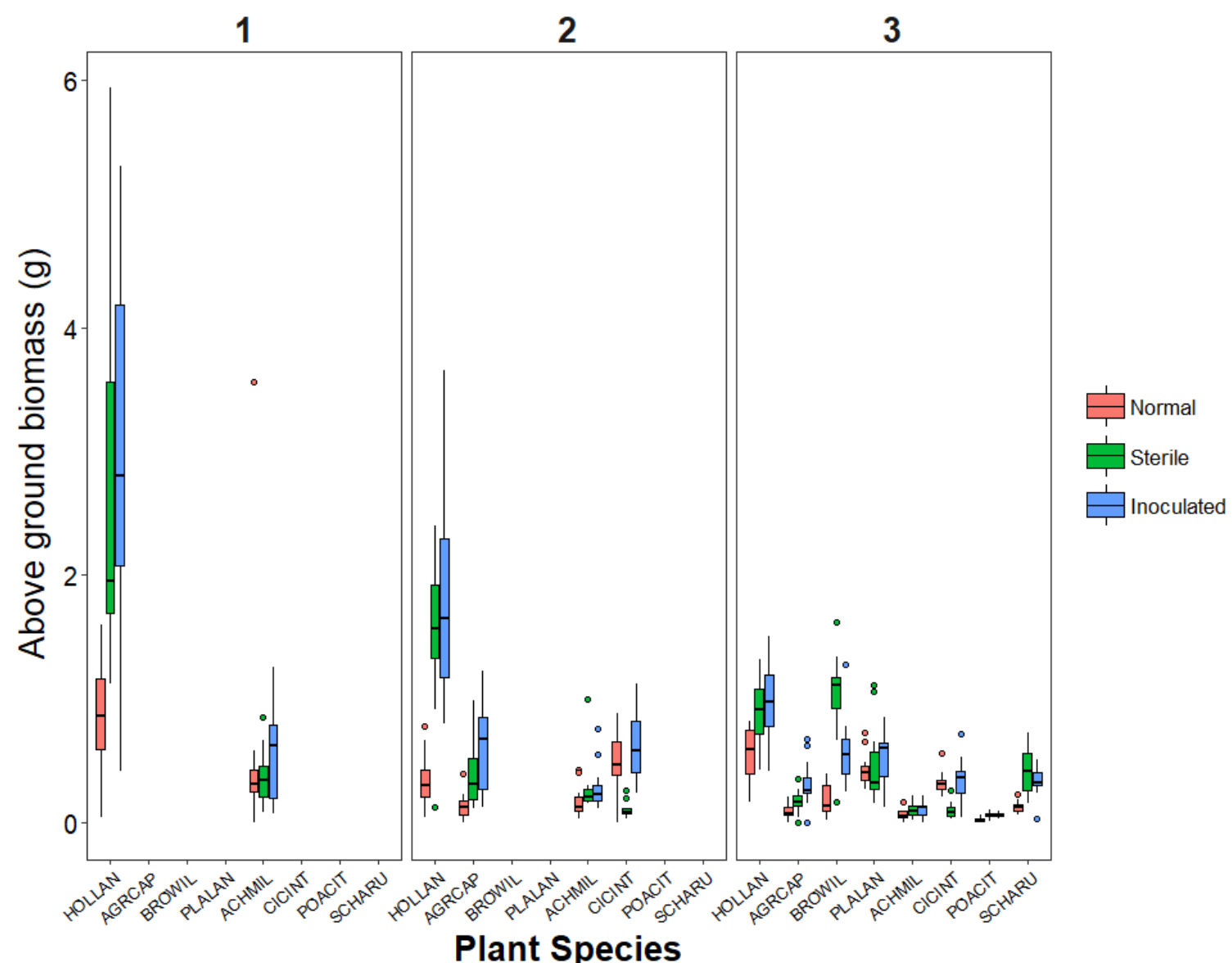

Figure 3. 11: Individual above ground biomass $(\mathrm{g})$ for each plant species in the environmental context experiment for initial communities. The fill shows the three different soil types. Plant species are shown here using the six-letter code. Letters 1, 2, and 3 represent the respective community treatments.

The late arrival individual biomass of $\mathrm{Hol}$ lan grown after the original community was only significantly affected by community type $\left(\mathrm{F}_{2,36}=11.9275, \mathrm{P}<0.001\right.$; Figure 3.12$)$. In community 1, Hol lan had significantly higher biomass than in both community 1 and 2 (Tukey post-hoc, $\mathrm{p}<0.001$; Figure 3.12). The individual Ach mil was significantly affected by community type $\left(F_{2,35}=7.7481, p<0.005\right.$; Figure 3.12$)$, soil type $\left(F_{2,35}=5.7726, p<0.005\right.$; Figure 3.12) and the interaction of community type and soil type $\left(\mathrm{F}_{4,35}=3.2659, \mathrm{p}<0.05\right.$; Figure 3.12). When grown in inoculated soil, there was a greater biomass in community 1 than community 2 and 3 (Tukey post-hoc, p<0.05; Figure 3.12). Only in community 1 did Ach 
mil show differences in biomass when in different soils. Both inoculated and normal soil was associated with greater plant biomass than sterile soil (Tukey post-hoc, $\mathrm{p}<0.05$; Figure 3.12).

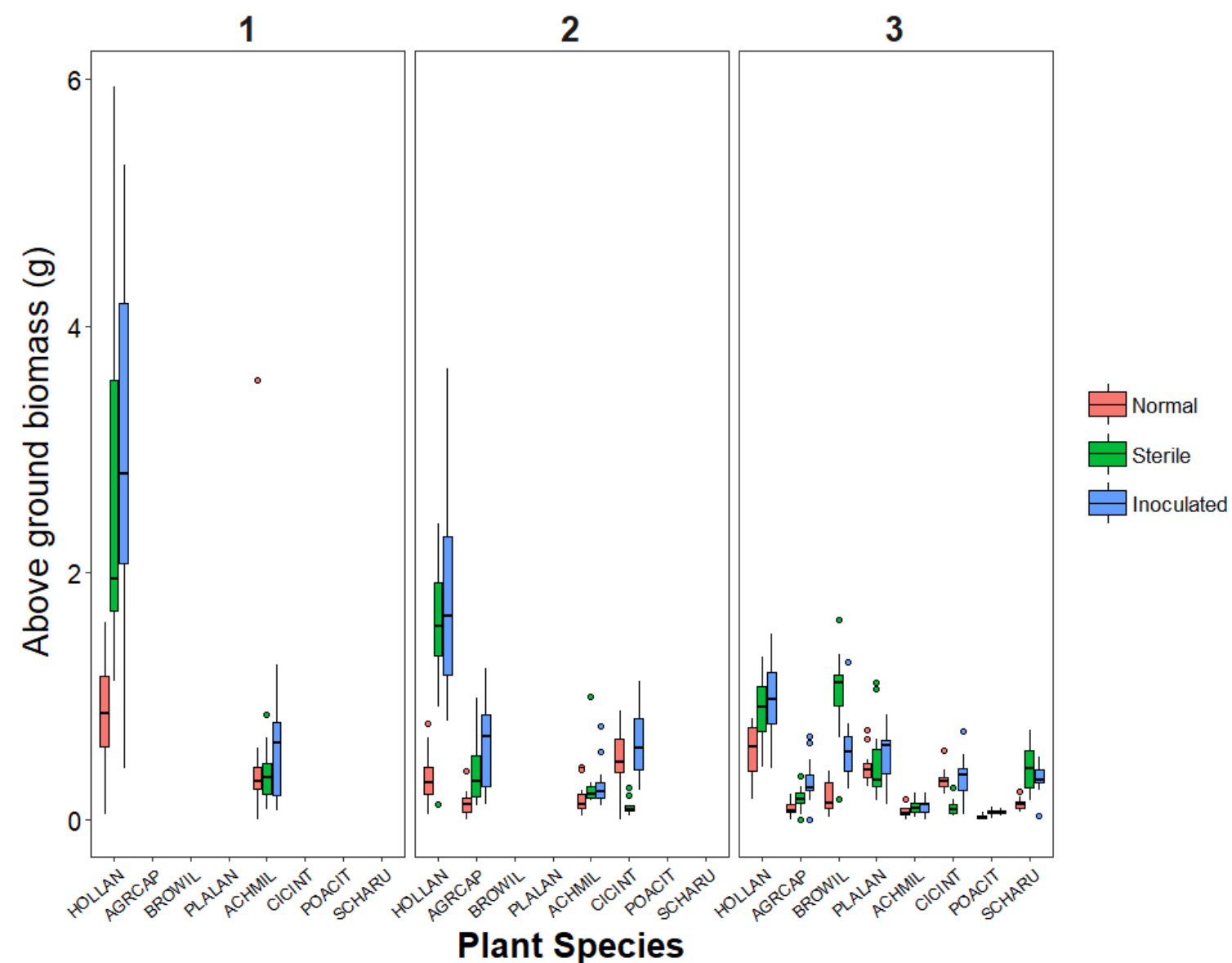

Figure 3. 12: Individual above ground biomass $(\mathrm{g})$ for each plant species in the environmental context experiment for late arrivals. The fill shows the three different soil treatments. Plant species are shown here using the six-letter code. Letters 1, 2, and 3 represent the respective community treatments. 
Chapter 4 - Discussion 


\section{Discussion}

This study evaluated the effect of plant interaction traits on the productivity of plant communities of varying species richness. Focusing on plant-AMF interactions, I selected plants that host many AMF species (generalist plants) and plants that host few AMF species (specialist plants). I tested the effects of plant interaction trait and species richness on various measures of productivity namely; NEE, total above and belowground plant biomass, and AMF biomass in soils and roots. I predicted that the addition of a specialist plant species would increase productivity relative to the addition of a generalist, but that this effect would be observable only at high levels of species richness, when plants are likely to compete for interaction partners. Although it is hard to draw definitive conclusions from the results gathered in this thesis, there are patterns in the results that align with my hypotheses and that are of interest for developing future studies.

\subsection{Productivity response to plant richness and interaction trait}

\subsubsection{Net ecosystem exchange}

I hypothesized that the addition of specialist plant species would lead to greater production (more negative NEE) than the addition of a generalist plant species to complex communities. I expected that this effect would be significant at higher species richness, so that the pattern of NEE by species richness treatment would be as follows; 3 plants $>5$ plants $>9$ plants. Overall, I found that NEE responded variably to the addition of plants with different interaction traits and was also variable between communities of different richnesses and over time. Overall runs, NEE was not affected by the addition of late arrivals (regardless of their interaction trait) and responded only to plant species richness. However, within runs, I found partial support for my hypotheses. For example, after 4 weeks of growth, the addition of an interaction specialist significantly increased carbon uptake (more negative NEE) in community 3 (9 plants) relative to community 2 ( 5 plants) or 1 ( 3 plants). Despite this, the results from Runs 1 ( 2 weeks after late arriving plants) and 3 (7 weeks after late arriving plants), did not conform to this pattern, and although differences in plant interaction traits and species richness drove some significant differences among treatments, these were idiosyncratic.

The results of Run 2 are interesting in light of research suggesting that the addition of an interaction specialist can increase niche complementarity and therefore have a positive effect 
on overall productivity. Loreau (2000) reviewed the literature on how plant biodiversity affects plant productivity and found two major mechanisms influencing positive short-term effects of species biodiversity on primary productivity. Mechanism 1 is the effect of functional niche complementarity, where trait variation in a community allow permanent associations, thus enhancing overall productivity. Mechanism 2 is the selection effect where the extreme trait values are selected for promoting dominance of the species with these extreme traits. Additionally, in local ecosystem sites, the effects of diversity are likely masked by the local environmental parameters (Loreau, 2000). My Run 2 results provide evidence in support of mechanism 1 and point to the importance of plant biodiversity in sustaining high production at baseline levels of fertility. Specifically, the specialists may lead to better utilisation of resources within the community (through niche-partitioning for resources) thus allowing for greater primary productivity (Catovsky et al., 2002).

While patterns of NEE were inconsistent overtime, it is interesting to note that in all three runs I found significant differences in NEE between the control and the specialist treatment. This suggests that plants with few interactions can have significant effects on the productivity of a plant community both positive and negative. While it is unclear what drove the inconsistent patterns of NEE overtime, some possible explanations could include increased nutrient loss from pots that were closest to the watering system, average ambient greenhouse temperatures over the growth period of the experiment. Light limitations and inconsistencies around the glasshouse could have been an issue to, however this was accounted for with randomisation of plant buckets once a fortnight.

The temporal differences in NEE to interaction trait may also relate to the size of late arrivals. For example, in Run 1, late arrival plants had only been in place for one week, they were still very small, and would not be expected to have a big effect on NEE. Rather, the significant differences in NEE observed in Run 1 are likely to reflect complex interactive effects, like competition for resources, happening within the already established peripheral community. This is highlighted by the significant interaction between plant richness and interaction trait that were present in NEE in Run1. For example, the addition of a specialist into a community with a greater plant species richness gave a more positive slope of carbon flux. This differs to results in Run2, where the same scenario resulted in a more negative slope of carbon flux. These temporal differences seen in Run 1 and later discussed in Run 2 and 3 suggests that in all runs there are multiple other factors, such as plant size, nutrient load, effecting NEE making it hard to disentangle where the effects of specialism or generalism are occurring. The potential of external factors contributing to the results in Run 1 make it difficult to disentangle and interpret the effects taking place in this run. 
It was expected that the patterns of NEE in Run 3 would be similar to those of Run 2.

Furthermore, in Run 3, I expected more negative NEE in the specialist treatments, relative to Run 2 because of their higher overall biomass, which would have supported greater carbon exchange. However, this was not what I observed. Rather, NEE was either unaffected (community 1) or increased (communities 2 and 3 ) by the addition of late arrivals in Run 3. This indicates that late arriving plants contributed more to respiration, than they did to photosynthesis by week 12, and suggests that the late arrivals, regardless of their interaction traits, were under stress by week 12 , which may indicate that they were not successfully recruiting to the plant community. Interestingly, late arriving specialists and generalists behaved differently under different levels of species richness in Run 3. In community 1, with only 3 species, late arrivals had no effect on NEE, in Run 2 with intermediate species richness (5 species) generalists caused NEE to become less negative (less productivity) relative to controls, while specialists did not. However, in community 3 with highest species richness, and where plants are expected to have competed most strongly for interaction partners, the addition of generalist and specialist plant species had similar, significant, effects on increasing (less negative) NEE. These results suggest that, under intermediate species richness, specialists did indeed affect increased productivity relative to generalists, that is, they did not reduce productivity as much as generalists did. Therefore, while contrary to expectations, the results of Run 3 do support the hypothesis that specialists may support greater production through their minimum competition for resources.

Across all NEE data, I observed significant effects of both run (number of weeks since establishment) and community type (species richness), indicating the strong effect of plant biomass in driving NEE. Nonetheless, these differences were smaller than I anticipated. Raich (1998) found that with an increase in above ground production there was also an increase in soil respiration. As species richness increased, Craine, Wedin, and Chapin (1999) found that microbial growth increased two fold where microbial respiration increased 1.5 fold. Burri, Niklaus, Grassow, Buchmann, and Kahmen (2018) found that increased soil respiration was directly related to an increased above ground production. They found a high annual above-ground productivity led to high rates of below-ground plant carbon allocation which in turn promoted soil respiration, either directly by root respiration or indirectly by increased availability of substrates for microbial respiration. Thus, these results support the variation in NEE data between runs seen in this experiment and also shed some possible light on why the carbon draw down in Run 3 was much less than expected even with increased biomass. 
In contrast to what was hypothesised, the absence of a relationship between interaction generalism and net ecosystem exchange in both Runs 1 and 3 agree with results gathered in a study looking at relationships between plant traits (leaf and root traits), soil properties and ecosystem carbon fluxes in field communities. De Long et al. (2019) found that in monocultures, plant traits (leaf and root traits) and soil properties can help predict carbon fluxes in these monoculture communities. However, in communities with mixed functional groups and functional traits, plant trait and soil properties were a bad predictor for carbon flux in mixed communities. Shoot biomass was the strongest predictor of carbon fluxes in both monocultures and mixed communities in their study. As mixed plant communities were used in our study, using plant traits, i.e., specialist or generalist for AMF, may not be important enough predictors of the carbon draw down. Instead, this previous research would suggest that total biomass of the plant community is the leading force behind community's carbon fluxes as plants are the primary source of carbon fixation via photosynthesis. Despite this, biomass still doesn't explain all the variation in the net ecosystem exchange data. For example, community 3 had the greatest biomass which theoretically should result in the greatest draw down of $\mathrm{CO}_{2}$ in community 3 Run 3, however this was not the case when compared with community 1,2 , where there was no significance in the slope of NEE between communities with respect to communities and their plant richness.

\subsubsection{Plant Biomass}

Analysis of the response of overall biomass to community type and plant interaction generalism identified that only community type had a significant effect. This did not fit my hypothesis as specialist and generalist traits of additional plants into the communities was hypothesised to influence overall community productivity. However, the results did support the hypothesis that with an increase in plants and species richness there would an increase in plant biomass. For above ground biomass there were between-community differences that found an increase in plant species richness lead to an overall increase in above ground productivity. The same trend was found for below ground biomass. As plant species richness and plant abundance increased so did overall below ground productivity. Both Mittelbach et al. (2001) and (Waide et al., 1999) found that there was a humped shaped correlation in many biodiversity and productivity studies where productivity increases to a point with richness then productivity will start to decline once the richness threshold in surpassed. From the biomass data gathered in this experiment, there is a definitive increase in overall productivity as species and plant richness increased. In studies that manipulate species richness via planting and removal, productivity was found to be a function of species richness (Hector et 
al., 1999; Tilman et al., 2001). The positive effect of species richness on productivity observed in these experiments is a result of either niche complementarity among species or higher probability of including large productive species in species-rich communities which links back to the two major mechanisms (previously discussed) that effect positive short-term effects of species biodiversity on primary productivity (Bischoff, Auge, \& Mahn, 2005; Loreau, 2000).

When comparing the difference between above and below ground, there was always more above ground biomass in a community than below ground biomass. As plant richness increased there was higher above and below ground biomass which fit the hypothesis made. In total biomass across communities there was greater allocation of above ground to below ground. Hollister and Flaherty (2010) found that in tundra communities two dominant graminoid plant species there was an increase in the above ground biomass over the below ground biomass when exposed to increased temperature. The difference in above and below ground allocation is expressed as a result in stressors like temperature, water, competition (light and below ground nutrients), and herbivory (Hollister \& Flaherty, 2010; Liira \& Zobel, 2000; Nie, Yang, Yang, \& Zhou, 2016). Nie et al. (2016) found that in communities with limiting factors plants generally allocate biomass to the organs that acquire the most limiting resource. From the time plants were planted into their respective communities right through to the time of harvest no nutrients were added to the experiment. With poor growth in the late arrival species this could be an indication of a limiting nutrient pool in the growth medium. This could have led to a greater allocation of above ground biomass as light become the more important limiting factor.

Because of the higher complementarity between species in a more species rich population, higher above ground biomass would have been anticipated in plants grown in communities containing higher functional richness where individual biomass decreases with increased richness. This expectation fitted the results found for each community; individual plant biomass decreased in response to increasing species richness despite maintaining greater overall community biomass. When the biomass of individual plants was assessed, the individual plant in the first arrival communities with the most biomass was the host interaction generalist plant Holcus lanatus. In community 3, Holcus lanatus had significantly higher biomass than all the other seven species but still a significantly lower biomass than when grown in community 1 . This was a trend for all species grown in both communities with low species richness and communities with high species richness. This pattern was also seen for Achillea millefolium and Cichorium intybus when grown in communities with lower plant richness. In a manipulative grassland experiment in conjunction with the Jena Experiment in 
Germany, Schmidtke, Rottstock, Gaedke, and Fischer (2010) found that individual plant species had decreased biomass in communities with greater species richness. They suggested their results were either due to an increase in plant density in more species-rich plots or due to the occurrence of species whose response opposes the average trend of decreasing individual aboveground biomass with increasing species richness. Plant height in the Jena Experiment was greater in individuals in higher species richness with a much greater leaf area index due to an increase in competition for light (Schmidtke et al., 2010). This suggests plants in species rich communities are greatly affected by competition and will put more energy into gaining light than into biomass density.

Interestingly in the first arrival communities Holcus lanatus had the greatest individual biomass across the three communities. However, in the "late arrival" plant species, Bromus willdenowii had the greatest individual biomass. Both plants are generalists and are dominant grassland species (Gatti, Ayala Torales, Cipriotti, \& Golluscio, 2013; Jones, Ostoja, Brooks, $\&$ Hutten, 2015). The differences seen between the two species at the different arrival times may be due to plant functional traits and nutrient load in the soil. As nutrient load would have been high at the start of the experiment Holcus lanatus may well out compete Bromus willdenowii. However, at the point of late arrival, nutrient load in the soil would have been much lower than at first planting. The fact that Bromus willdenowii grew better than Holcus lanatus at lower nutrient levels may indicate the difference in plants ability to dominate when certain limiting factors like nutrient load are present.

The composition of the plant communities present in this study, may also impact individual plant productivity and ultimately community biomass. The effects of grasses and herbs growing together were observed in the Jena Experiment and results concluded that grasses were found to negatively impact the biomass of non-grasses in shared plots (Roscher et al., 2004). Schmidtke et al. (2010) concluded similar results and found that grasses had a negative impact on individual productivity of non-grass species when grown in the same communities. Grasses are better competitors than forbs, requiring a larger amount of phosphorous and water as well as better at nitrogen acquisition (Fargione, Brown, \& Tilman, 2003). This supported our findings, as grasses were the dominant functional group throughout this experiment with Holcus lanatus having the greatest biomass across communities. Individual plant productivity is greatly impacted by the diversity of the community it exists in and the direction the effect of community plant richness has is very different between individual species. Schmidtke et al. (2010) suggests that "diversity of the surrounding community needs to be taken into account when interpreting drivers of the performance of individual plants." As the study in this thesis is a manipulative one, the plants were chosen on their interaction generality and were known 
to be either host generalist or specialists to AMF fungi. This allowed the choice of dominant species and less dominant species to be chosen to create complex communities. The outcome of the biomass results points to both niche complementary due to the complex communities grown allowing greater biomass in a higher species richness community, and to the selection of known generalist species, which are known to be more dominant.

\subsubsection{NLFA}

The addition of an interaction specialist and generalist plant to a complex community showed no effect on overall community AMF biomass. A known biomarker in AMF was used to quantify the concentration of NLFA in both soil and roots to give a proxy for overall AMF biomass. Although the hypothesis was not supported for interaction generalism, there was a significant effect of increasing plant species richness on NLFA in plant roots. There was no difference in NLFA concentration in soil across the community types, however as plant species richness increased, there was an increase in the NLFA concentration in roots. The total AMF biomass in total soil may not show any relationship with increased plant species richness due to the sampling methods and the overall volume Wang et al. (2019) explored diversity-productivity relationships in late successional plant communities and found that positive diversity-productivity relationships were directly related to more diverse microbial communities in soil and roots. A more diverse belowground microbial community can supress pathogen effects on the plant species and increase microbial resource partitioning allowing for overall greater plant species richness. Landis, Gargas, and Givnish (2004) produced a study looking at sites and microsites across a Midwestern American savannah and discovered that the numbers of plant and AMF species per sample were positively correlated. Y. Lekberg, Gibbons, Rosendahl, and Ramsey (2013) found no relationship between plant species richness and fungal OTU (operational taxonomic unit) numbers but a good correlation between AMF colonization and fungal OTU numbers. They suggest that the "positive relationship between plant and AMF richness is not always strong and that carbon allocation could be one factor that determines AMF richness", and concluded more experimental work with altered carbon allocation to AMF is required to test this hypothesis and exclude alternative explanations. Many studies have found an increase in AMF richness in highly diverse plant communities due to a greater potential AMF niche space (Landis et al., 2004; Y. Lekberg et al., 2013; Urcelay \& Díaz, 2003). However this is a contentious issue and not supported throughout the literature (Borstler, Renker, Kahmen, \& Buscot, 2006; Johnson et al., 2004; Urcelay \& Díaz, 2003). The inconsistency in the support of AMF diversity throughout the literature in relation to plant species diversity indicates factors other than plant diversity are also important in 
structuring AMF communities (Antoninka et al., 2011). The results of this thesis show a clear increase in AMF biomass as species richness increases which supports the idea that an increase in plant community diversity, leads to greater AMF niche space.

\subsection{Environmental context}

\subsubsection{Net ecosystem exchange}

It was hypothesised that in sterile soil there would have been a negative relationship between soil type and carbon drawdown, inoculated soil would have had a slightly more positive relationship, whereas normal soil would have a positive relationship with carbon drawdown. In contrast to this original hypothesis, across the three soil types the drawdown of carbon had a more positive relationship (more negative slope of carbon flux) with both sterile and inoculated soils compared to normal soil. The effect of plant species richness shows that with an increase in plant species there is a trend in carbon flux as more negative slopes of carbon flux were present in both normal and sterile soils. The differences seen in these results reflect the findings in the plant biomass results for these two different soil types. The inoculated soil results are harder to interpret as there is significantly more carbon drawdown compared to normal soil in Run 2, however in Run 3 this can't be concluded. With an increase in plant species richness there is a negative relationship with the slope of carbon flux. Both sterile and inoculated soil had higher plant biomass than normal soils and Run 2 proves this with larger drawdowns of carbon in communities 2. Run 3 gave unexpected results as the slope of carbon flux in both sterile and inoculated soils was less negative which shows there was either greater overall community respiration taking place or less photosynthesis or both. Across the different soil types, community plant richness seems to be the strongest indicator of where the effect of soil type occurs.

\subsubsection{Plant Biomass}

There was a significant increase in plant biomass in both sterile and inoculated soils compared to normal soils which is contrary to the hypothesis as sterile soil was expected to negatively impact overall plant biomass, and inoculated soil was only going to regain partial biomass lost, in overall biomass both above and below ground. This hypothesis was based on the idea that AMF would positively impact plant biomass and soil sterilisation acted as a control to remove AMF from the soil. Interestingly, de Deyn, Raaijmakers, and van der Putten (2004) found that in sterile soils compared to non-sterile soils biomass in communities 
with no added nutrients, biomass was almost three times higher after 4 months of growth. They found that grasses were much more dominant in sterile soils and forb abundance decreased as the experiment grew. This thesis found that the biomass of individual plants was highest in grasses in both sterile and inoculated soils. All of the forbs excluding Plantago lanceolata had lower levels of biomass in sterile and inoculated soils. Plantago lanceolata had a greater biomass compared to the other forbs but this may be a result of other functional traits as it is an interaction generalist so may have done better in inoculated soil as it can occupy more AMF niche space. Another important factor affecting the relationship between soil sterility and plant biomass is the short interval of this experiment as plants were harvested just before flowering. In the early stages of AMF network formations, plants invest high energy inputs into these plant/fungus relationships and gain very little return from these early relationships (Ardestani, Jílková, Bonkowski, \& Frouz, 2019). The time taken to create good interactions between plants and fungi may characterise the above and below ground biomass results in this experiment contributing to the effect of AMF vs no AMF, i.e, sterilised, soils.

The high plant biomass found in sterile soils could also likely be linked to the sterilisation process. After the sterilisation process, biomass was found to be greater in both sterile and inoculated soils. This was contrary to the hypothesis and conclusions could be made there was an increase in nutrients due to dead microbial biomass in sterilized soil due to the sterilisation method (Semchenko et al., 2018). Throughout the literature, soil sterilisation is constantly an issue that doesn't seem to have been resolved. Soka and Ritchie (2016) used a similar steam pasteurising method and found biomass was higher in non-sterilised soils. de Deyn et al. (2004) used a gamma irradiation method and found that grasses were much more dominant in sterilised soils than in non-sterile soil and shot biomass was greater throughout the experiment in sterile soils. Ardestani et al. (2019) sterilised soil using a gamma irradiation technique and found that there was a negative effect of AMF on above and below ground biomass compared to sterile soils. Their study however, only used a single AMF species so positive effects may still have occurred with a more diverse AMF community. Semchenko et al. (2018) found that in conspecific soils that were sterilised using gamma irradiation and not sterilised, plants grew on average 2.8 times bigger in the sterilised soil. Wolf, Dao, Scott, and Lavy (1989) concluded that sterilisation using Cobalt-60 irradiation, propylene oxide, mercuric chloride, and autoclaving $2 \mathrm{X}$ or $3 \mathrm{X}$, effectively eliminated the microbial community in the soils they were testing. The results found here and in other studies sharing similar sterilisation processes provide a good platform for future methodological studies to determine what soil sterilisation processes are needed for effective removal of the microbial community and to further elucidate the effects of soil sterilisation process on plant communities. 


\subsubsection{NLFA}

Echoing the results of plant productivity in the Environmental context experiment, there was also increased NLFA in roots in both sterile and inoculated soils compared to normal soils. When we consider the AMF biomass in both sterile and inoculated soil types, this suggests two scenarios; sterilisation had no impact on pre-existing AMF communities in the soil or the pre-existing AMF community in the soil was negligible to start with, hence sterilisation had no notable effects. This would suggest that sterilisation was having a greater impact on other components of the soil community (i.e. bacteria etc) that was then affecting the ability of AMF communities to establish on roots. In sterilised soils, it seemed that AMF biomass on roots was able to establish and colonise to a higher degree than in normal soils. The NLFA results fit the NEE results with respect to the respiration of the microbial community. With a higher biomass of AMF there should be an increase in soil respiration (Pietikåinen, Pettersson, \& Bååth, 2005), which would lead to a more positive slope of carbon flux in both the sterile and inoculated soils. Despite Pietikåinen et al. (2005) identifying this increase in response to soil rather than root associated NLFA, the role of root NLFA may be similar especially in an environmental context of sterile soils.

\subsection{Experimental method limitations}

\subsubsection{Net ecosystem exchange}

Due to the large variability in the NEE results from both experiments, there are a number of method limitations that can be addressed and may account for this variability. Primarily, the resolution in the NEE output was not as sensitive as was expected. This was largely related to

NEE measurement chamber design and measurement conditions. Despite the chamber used to measure the carbon draw down being greatly reduced in size to maximise sensitivity, this reduction, and ultimately sensitivity, was limited by temperature control issues impacting plant viability. Soil respiration responds differently to increased temperatures. The Q10 temperature coefficient is a measure of the rate of change of a biological or chemical system as a consequence of increasing the temperature by $10{ }^{\circ} \mathrm{C}$ (the coefficient for the exponential relationship between soil respiration and temperature, multiplied by 10) (Reyes, Pendergast, \& Yamazaki, 2008). The instantaneous soil respiration rate often increases with temperature up to around $40{ }^{\circ} \mathrm{C}$ or more, even in soils from cold climates (Pietikåinen et al., 2005). The Q10 for autotrophic root respiration and rhizosphere decomposition was higher than respiration of bulk soil and soil lacking roots (Boone, Nadelhoffer, Canary, \& Kaye, 1998). In 
the Environmental context experiment, there was a higher below ground biomass in both sterile soils and inoculated soils indicating the Q10 of the autotrophic root respiration and rhizosphere could have explained the slope of carbon flux, NEE, in Run 3 had a stronger negative relationship in sterile and inoculated soil. In addition to this, each community was grown in seven litres of grown medium, which occupied a large volume of space in the NEE chamber. As NEE measurements record the total carbon flux in a system, the large volumes of growth medium to overall plant biomass in each community may well have created a great imbalance in the ecosystem draw down of carbon versus the ecosystem respiration rate.

Temperature limitations were prevalent in the experimental location. As the experiment spanned a 3-month period the temperature in the glasshouse may have been higher during the last run of three runs. The ambient temperature of the glasshouse was never recorded. The average temperatures recorded in the NEE chamber across all the runs was different by $1{ }^{\circ} \mathrm{C}$. However, within each run there was a significant temperature difference between treatments and the control treatments. Post hoc runs showed that for all runs, all communities with both specialists and generalists planted as late arrivals had significantly more variation in temperature across all runs compared to the control communities. The temperature range in the controls was very small compared with the specialists and generalists. This was due to sampling errors. Pietikåinen et al. (2005) concluded that fungal and bacterial growth rates were affected differently by temperature and the optimum activity rates of fungi were at temperatures between 25 and $30^{\circ} \mathrm{C}$ which was the same for the optimal activity rates of bacteria. Links between temperature and soil microbe activity may well have been influencing the NEE results in both experiments. Without ambient growth temperatures throughout the experiment understanding these results is difficult.

Leaf area can have large impacts on the respiration and photosynthetic rate of plant species. Poorter (1993) measured the growth response to elevated CO2 of 10 species and found that photosynthesis expressed on a leaf area basis was enhanced by $20 \%$. Leaf respiration in the light and leaf respiration in the dark increased with increasing leaf temperature (Way \& Yamori, 2014). Leaf respiration in the dark will often increase with a linear rise in leaf temperature (Way \& Yamori, 2014). There are few measurements taken on the effects of temperature on leaf respiration in the light, so most data collected is extrapolated from leaf respiration in the dark. With greater overall biomass in both sterile and inoculated soils this would indicate greater leaf biomass which would result in greater leaf respiration. This may well have impacted the more positive slopes of carbon flux in Run 3 for both sterile and inoculated soil. 


\subsubsection{Biomass}

The overall hypothesis of this thesis was that the addition of an interaction specialist into a complex community would increase overall productivity of the community. After the initial complex communities were grown and well established, a late arrival generalist or specialist plant was added. The delayed timing in adding late arrival plants, may have impacted the successful growth of these plants, contributing the effects these plants may have had on the surrounding plant community. Specifically, there was limited growth seen in second arrival plants from second arrival planting time to plant harvest. Furthermore, there were poor growth rates for all the generalist and specialists planted second. The initial communities all grew reasonably well with an increase in individual plant biomass throughout the experiment. The time period between first planting and second planting had a large effect on the final productivity of late arrival plants. This could well have been due to lack of nutrient load in the soil once an initial community had utilised most of it. It is known that with an increase in species richness there is also an increase in light competition (Schmidtke et al., 2010). Plants put more effort into growing taller to gain as much light as possible when in the presence of other competitor and put less energy in to abundance. With an increase in competition, not just for light, but for nutrients, space, and interaction partners in the soil, the differences in biomass results seen in this experiment may well have been to do with the increased pressure put on individual plants as species richness increased in communities. P. Kardol, Souza, and Classen (2013) found that arrival times of species had a large effect. However, the size of this effect was determined by the nutrient availability. At increased planting intervals there were larger differences between control communities and communities planted later with stronger differences at higher nutrient supply. They suggest at higher nutrient loads, early arrival plant species acquire light resources more efficiently, preventing the successful establishment of late arrival species even at short planting intervals.

Using monocultures to observe differences in each plant species may well be useful when trying to determine the effects of species richness and plant abundance on the specific functional traits of each species. Niche complementarity and niche competition was never looked at in this experiment with regard to functional groups and functional traits of each species. Using the mixture of forbs and grasses present in this study and looking at speciesspecific abilities to respond to the addition of interaction generalist or specialist into either a monoculture or an already established community could be used to determine the different consequences for niche partitioning among species and among soil interaction partners. Lipowsky et al. (2015) discusses that functional complementarity among species may be due to a plants ability to maintain an increased plasticity in higher species richness's that is not 
seen in trait values in monocultures. Or contrastingly, large plasticity in functional traits may lessen in higher richness's, as species adjust to become more similar. These differences may play an integral role in the effects of adding different interaction generalist and specialist species into already complex communities.

\subsection{Conclusion}

This study identified that the addition of interaction generalism into already complex communities may influence overall productivity particularly their level of carbon drawdown. There was a strong effect of community plant richness across the productivity measures, and this looked to be the driving factor when it comes to many significant differences in productivity responses. This study highlights the importance of species richness and plant abundance on the amount of above and below ground productivity in a community. Additionally, NLFA of AMF in roots significantly increased as species richness and abundance increased. The results of the Environmental context experiment were unexpected but gave insight into the need to investigate effective soil sterilisation methods. As the idea of interaction generalism is still in its scientific infancy, there is great scope for repeats and manipulation of studies like this to find out how AMF interactions impact plant community ecology. 


\section{References}

Abraham, J. K., Corbin, J. D.\& D'Antonio, C. M. (2009). California native and exotic perennial grasses differ in their response to soil nitrogen, exotic annual grass density, and order of emergence. Plant Ecology, 201, 445-456.

Adler, P. B., Seabloom, E. W., Borer, E. T., Hillebrand, H., Hautier, Y., Hector, A., . . Yang, L. H. (2011). Productivity is a poor predictor of plant species richness. Science, 333, 1750-1753.

Almany, G. R. (2004). Priority effects in coral reef fish communities of the great barrier reef. Ecology, 85, 2872-2880.

Antoninka, A., Reich, P. B.\& Johnson, N. C. (2011). Seven years of carbon dioxide enrichment, nitrogen fertilization and plant diversity influence arbuscular mycorrhizal fungi in a grassland ecosystem. The New Phytologist, 192, 200-214.

Ardestani, M. M., Jílková, V., Bonkowski, M.\& Frouz, J. (2019). The effect of arbuscular mycorrhizal fungi rhizophagus intraradices and soil microbial community on a model plant community in a post-mining soil. Plant Ecology, 220, 789-800.

Baeten, L., Bruelheide, H., van der Plas, F., Kambach, S., Ratcliffe, S., Jucker, T., . . SchererLorenzen, M. (2019). Identifying the tree species compositions that maximize ecosystem functioning in european forests. Journal of Applied Ecology, 56, 733-744.

Balser, T. C., Treseder, K. K.\& Ekenler, M. (2005). Using lipid analysis and hyphal length to quantify am and saprotrophic fungal abundance along a soil chronosequence. Soil Biology \& Biochemistry, 37, 601-604.

Bates, D., Mächler, M., Bolker, B.\& Walker, S. (2015). Fitting linear mixed-effects models using Ime4. 2015, 67, 48.

Batten, K. M., Scow, K. M., Davies, K. F.\& Harrison, S. P. (2006). Two invasive plants alter soil microbial community composition in serpentine grasslands. Biological Invasions, 8 , 217-230.

Batten, K. M., Scow, K. M.\& Espeland, E. K. (2008). Soil microbial community associated with an invasive grass differentially impacts native plant performance. Microbial Ecology, 55, 220-228.

Bischoff, A., Auge, H.\& Mahn, E. (2005). Seasonal changes in the relationship between plant species richness and community biomass in early succession. Basic and Applied Ecology, 6, 385-394.

Bligh, E. G.\& Dyer, W. J. (1959). A rapid method of total lipid extraction and purification. Canadian Journal of Biochemistry and Physiology, 37, 911-917.

Boone, R. D., Nadelhoffer, K. J., Canary, J. D.\& Kaye, J. P. (1998). Roots exert a strong influence on the temperature sensitivityof soil respiration. Nature, 396, 570-572.

Borstler, B., Renker, C., Kahmen, A.\& Buscot, F. (2006). Species composition of arbuscular mycorrhizal fungi in two mountain meadows with differing management types and levels of plant biodiversity. Biology and Fertility of Soils, 42, 286-298.

Breza, L. C., Souza, L., Sanders, N. J.\& Classen, A. T. (2012). Within and between population variation in plant traits predicts ecosystem functions associated with a dominant plant species. Ecology and Evolution, 2, 1151-1161.

Burri, S., Niklaus, P. A., Grassow, K., Buchmann, N.\& Kahmen, A. (2018). Effects of plant productivity and species richness on the drought response of soil respiration in temperate grasslands. Plos One, 13, 18.

Buyer, J. S.\& Sasser, M. (2012). High throughput phospholipid fatty acid analysis of soils. Applied Soil Ecology, 61, 127-130.

Callaway, R. M. (1995). Positive interactions among plants. Botanical Review, 61, 306-349. 
Cardinale, B. J., Wright, J. P., Cadotte, M. W., Carroll, I. T., Hector, A., Srivastava, D. S., . . Weis, J. J. (2007). Impacts of plant diversity on biomass production increase through time because of species complementarity. Proceedings of the National Academy of Sciences of the United States of America, 104, 18123-18128.

Carey, C. J., Blankinship, J. C., Eviner, V. T., Malmstrom, C. M.\& Hart, S. C. (2017). Invasive plants decrease microbial capacity to nitrify and denitrify compared to native california grassland communities. Biological Invasions, 19, 2941-2957.

Catovsky, S., Bradford, M. A.\& Hector, A. (2002). Biodiversity and ecosystem productivity: Implications for carbon storage. Oikos, 97, 443-448.

Chagnon, P. L., Bradley, R. L., Maherali, H.\& Klironomos, J. N. (2013). A trait-based framework to understand life history of mycorrhizal fungi. Trends Plant Sci, 18, 484491.

Chapin iii, F. S., A., M. P.\& A., M. H. (2002). Principles of terrestrial ecosystem ecology. In: New York: Springer-Verlag.

Cheng, Y. X., Zhang, C. Y., Zhao, X. H.\& von Gadow, K. (2018). Biomass-dominant species shape the productivity-diversity relationship in two temperate forests. Annals of Forest Science, 75, 9.

Craine, J. M., Wedin, D. A.\& Chapin, F. S. (1999). Predominance of ecophysiological controls on soil co2 flux in a minnesota grassland. Plant and Soil, 207, 77-86.

Davidson, A. M., Jennions, M.\& Nicotra, A. B. (2011). Do invasive species show higher phenotypic plasticity than native species and, if so, is it adaptive? A meta-analysis. Ecology Letters, 14, 419-431.

De Deyn, G. B., Cornelissen, J. H. C.\& Bardgett, R. D. (2008). Plant functional traits and soil carbon sequestration in contrasting biomes. Ecology Letters, 11, 516-531.

de Deyn, G. B., Raaijmakers, C. E.\& van der Putten, W. H. (2004). Plant community development is affected by nutrients and soil biota. Journal of Ecology, 92, 824-834.

De Jong, M.\& Leyser, O. (2012). Developmental plasticity in plants. Paper presented at the Cold Spring Harbor symposia on quantitative biology.

De Long, J. R., Jackson, B. G., Wilkinson, A., Pritchard, W. J., Oakley, S., Mason, K. E., . . . Bardgett, R. D. (2019). Relationships between plant traits, soil properties and carbon fluxes differ between monocultures and mixed communities in temperate grassland. Journal of Ecology, 107, 1704-1719.

Demmig-Adams, B.\& Adams, W. W. (2006). Photoprotection in an ecological context: The remarkable complexity of thermal energy dissipation. New Phytologist, 172, 11-21.

Deslippe, J. R., Tylianakis, J.\& Hartmann, M. (2016). First come, best served? The role of generalist and specialist species in the assembly, diversity and productivity of ecosystems. . Royal Society of New Zealand Marsden Fast Start Grant, \$300,000.

Dickie, I. A., Bufford, J. L., Cobb, R. C., Desprez-Loustau, M. L., Grelet, G., Hulme, P. E., . . . Williams, N. M. (2017). The emerging science of linked plant-fungal invasions. New Phytologist, 215, 1314-1332.

Dickson, T. L., Hopwood, J. L.\& Wilsey, B. J. (2012). Do priority effects benefit invasive plants more than native plants? An experiment with six grassland species. Biological Invasions, 14, 2617-2624.

Duarte, L. D. S., Debastiani, V. i. J., Carlucci, M. B.\& Diniz-Filho, J. A. F. (2018). Analyzing community-weighted trait means across environmental gradients: Should phylogeny stay or should it go? Ecology, 99, 385-398.

Encyclopædia Britannica, i. (Producer). (2018, 30 May 2018). The carbon cycle. [Digital image] Retrieved from <https://www.britannica.com/science/carbon-cycle>

Engelmoer, D. J. P.\& Kiers, E. T. (2015). Host diversity affects the abundance of the extraradical arbuscular mycorrhizal network. New Phytologist, 205, 1485-1491. 
Fargione, J., Brown, C. S.\& Tilman, D. (2003). Community assembly and invasion: An experimental test of neutral versus niche processes. Proceedings of the National Academy of Sciences, 100, 8916-8920.

Frostegard, A.\& Baath, E. (1996). The use of phospholipid fatty acid analysis to estimate bacterial and fungal biomass in soil. Biology and Fertility of Soils, 22, 59-65.

Fukami, T. (2015). Historical contingency in community assembly: Integrating niches, species pools, and priority effects. In D. J. Futuyma (Ed.), Annual review of ecology, evolution, and systematics, vol 46 (Vol. 46, pp. 1-23). Palo Alto: Annual Reviews.

Fukami, T., Bezemer, T. M., Mortimer, S. R.\& van der Putten, W. H. (2005). Species divergence and trait convergence in experimental plant community assembly. Ecology Letters, 8, 1283-1290.

Garnier, E.\& Navas, M. L. (2012). A trait-based approach to comparative functional plant ecology: Concepts, methods and applications for agroecology. A review. Agronomy for Sustainable Development, 32, 365-399.

Gatti, M. L., Ayala Torales, A. T., Cipriotti, P. A.\& Golluscio, R. A. (2013). Leaf and tiller dynamics in two competing c 3 grass species: Influence of neighbours and nitrogen on morphogenetic traits. Grass and Forage Science, 68, 151-164.

Gioria, M.\& Pysek, P. (2017). Early bird catches the worm: Germination as a critical step in plant invasion. Biological Invasions, 19, 1055-1080.

Goodale, K. M.\& Wilsey, B. J. (2018). Priority effects are affected by precipitation variability and are stronger in exotic than native grassland species. Plant Ecology, 219, 429439.

Grace, J. B., Anderson, T. M., Seabloom, E. W., Borer, E. T., Adler, P. B., Harpole, W. S., . . Smith, M. D. (2016). Integrative modelling reveals mechanisms linking productivity and plant species richness. Nature, 529, 390.

Greipsson, S.\& DiTommaso, A. (2006). Invasive non-native plants alter the occurrence of arbuscular mycorrhizal fungi and benefit from this association. Ecological Restoration, 24, 236-241.

Grman, E.\& Suding, K. N. (2010). Within-year soil legacies contribute to strong priority effects of exotics on native california grassland communities. Restoration Ecology, 18, 664-670.

Guo, Q. F.\& Berry, W. L. (1998). Species richness and biomass: Dissection of the humpshaped relationships. Ecology, 79, 2555-2559.

Hattenschwiler, S. (2001). Tree seedling growth in natural deep shade: Functional traits related to interspecific variation in response to elevated co2. Oecologia, 129, 31-42.

Hector, A., Schmid, B., Beierkuhnlein, C., Caldeira, M. C., Diemer, M., Dimitrakopoulos, P. G., ... Lawton, J. H. (1999). Plant diversity and productivity experiments in european grasslands. Science, 286, 1123-1127.

Hollister, R. D.\& Flaherty, K. J. (2010). Above- and below-ground plant biomass response to experimental warming in northern alaska. Applied Vegetation Science, 13, 378-387.

Hulshof, C. M.\& Swenson, N. G. (2010). Variation in leaf functional trait values within and across individuals and species: An example from a costa rican dry forest. Functional Ecology, 24, 217-223.

Johansen, A., Finlay, R. D.\& Olsson, P. A. (1996). Nitrogen metabolism of external hyphae of the arbuscular mycorrhizal fungus glornus intraradices. New Phytologist, 133, 705712.

Johnson, D., Vandenkoornhuyse, P. J., Leake, J. R., Gilbert, L., Booth, R. E., Grime, J. P., . . . Read, D. J. (2004). Plant communities affect arbuscular mycorrhizal fungal diversity and community composition in grassland microcosms. New Phytologist, 161, 503515. 
Jones, L. J., Ostoja, S. M., Brooks, M. L.\& Hutten, M. (2015). Short-term response of holcus lanatus I. (common velvetgrass) to chemical and manual control at yosemite national park, USA. Invasive Plant Science and Management, 8, 262-268, 267.

Jung, S. C., Martinez-Medina, A., Lopez-Raez, J. A.\& Pozo, M. J. (2012). Mycorrhiza-induced resistance and priming of plant defenses. Journal of Chemical Ecology, 38, 651-664.

Kajimoto, T., Osawa, A., Usol'tsev, V.\& P. Abaimov, A. (2010). Biomass and productivity of siberian larch forest ecosystems (Vol. 209).

Kamruzzaman, M., Ahmed, S.\& Osawa, A. (2017). Biomass and net primary productivity of mangrove communities along the oligohaline zone of sundarbans, bangladesh. Forest Ecosystems, 4, 9.

Kardol, P., Cornips, N. J., van Kempen, M. M. L., Bakx-Schotman, J. M. T.\& van der Putten, W. H. (2007). Microbe-mediated plant-soil feedback causes historical contingency effects in plant community assembly. Ecological Monographs, 77, 147-162.

Kardol, P., Souza, L.\& Classen, A. T. (2013). Resource availability mediates the importance of priority effects in plant community assembly and ecosystem function. Oikos, 122, 84-94.

Kirwan, L., Connolly, J., Finn, J. A., Brophy, C., Luscher, A., Nyfeler, D.\& Sebastia, M. T. (2009). Diversity-interaction modeling: Estimating contributions of species identities and interactions to ecosystem function. Ecology, 90, 2032-2038.

Klironomos, J. N. (2002). Feedback with soil biota contributes to plant rarity and invasiveness in communities. Nature, 417, 67-70.

Klironomos, J. N. (2003). Variation in plant response to native and exotic arbuscular mycorrhizal fungi. Ecology, 84, 2292-2301.

Klironomos, J. N., McCune, J., Hart, M.\& Neville, J. (2000). The influence of arbuscular mycorrhizae on the relationship between plant diversity and productivity. Ecology Letters, 3, 137-141.

Landis, F. C., Gargas, A.\& Givnish, T. J. (2004). Relationships among arbuscular mycorrhizal fungi, vascular plants and environmental conditions in oak savannas. New Phytologist, 164, 493-504.

Lauenroth, W. K.\& Sala, O. E. (1992). Long-term forage production of north american shortgrass steppe. Ecol Appl, 2, 397-403.

Lavorel, S.\& Garnier, E. (2002). Predicting changes in community composition and ecosystem functioning from plant traits: Revisiting the holy grail. Functional Ecology, 16, 545556.

Lekberg, Y., Gibbons, S. M., Rosendahl, S.\& Ramsey, P. W. (2013). Severe plant invasions can increase mycorrhizal fungal abundance and diversity. Isme Journal, 7, 1424-1433.

Lekberg, Y.\& Helgason, T. (2018). In situ mycorrhizal function - knowledge gaps and future directions. New Phytologist, 220, 957-962.

Leopold, D. R., Tanentzap, A. J., Lee, W. G., Heenan, P. B.\& Fukami, T. (2015). Evolutionary priority effects in new zealand alpine plants across environmental gradients. Journal of Biogeography, 42, 729-737.

Li, Y., Dong, S. K., Liu, S. L., Su, X. K., Wang, X. X., Zhang, Y., .. . Tang, L. (2019). Relationships between plant diversity and biomass production of alpine grasslands are dependent on the spatial scale and the dimension of biodiversity. Ecological Engineering, 127, 375-382.

Liira, J.\& Zobel, K. (2000). The species richness-biomass relationship in herbaceous plant communities: What difference does the incorporation of root biomass data make? Oikos, 91, 109-114.

Lipowsky, A., Roscher, C., Schumacher, J., Michalski, S. G., Gubsch, M., Buchmann, N., . . Schmid, B. (2015). Plasticity of functional traits of forb species in response to biodiversity. Perspectives in Plant Ecology, Evolution and Systematics, 17, 66-77. 
Loreau, M. (2000). Biodiversity and ecosystem functioning: Recent theoretical advances. Oikos, 91, 3-17.

Loreau, M.\& Hector, A. (2001). Partitioning selection and complementarity in biodiversity experiments. Nature, 412, 72-76.

Mari, M., Silje, B., John, D., Maarja, Ö., Riccardo, B., Helge, B., . . Gian-Reto, W. (2011). Alien plants associate with widespread generalist arbuscular mycorrhizal fungal taxa: Evidence from a continental-scale study using massively parallel 454 sequencing. Journal of Biogeography, 38, 1305-1317.

Mason, N. W. H., Mouillot, D., Lee, W. G.\& Wilson, J. B. (2005). Functional richness, functional evenness and functional divergence: The primary components of functional diversity. Oikos, 111, 112-118.

Mayfield, M. M., Bonser, S. P., Morgan, J. W., Aubin, I., McNamara, S.\& Vesk, P. A. (2010). What does species richness tell us about functional trait diversity? Predictions and evidence for responses of species and functional trait diversity to land-use change. Global Ecology and Biogeography, 19, 423-431.

Mittelbach, G. G., Steiner, C. F., Scheiner, S. M., Gross, K. L., Reynolds, H. L., Waide, R. B., . . Gough, L. (2001). What is the observed relationship between species richness and productivity? Ecology, 82, 2381-2396.

Moora, M., Berger, S., Davison, J., Öpik, M., Bommarco, R., Bruelheide, H., ... Walther, G. R. (2011). Alien plants associate with widespread generalist arbuscular mycorrhizal fungal taxa: Evidence from a continental-scale study using massively parallel 454 sequencing. Journal of Biogeography, 38, 1305-1317.

Mummey, D. L.\& Rillig, M. C. (2006). The invasive plant species centaurea maculosa alters arbuscular mycorrhizal fungal communities in the field. Plant and Soil, 288, 81-90.

Nie, X. Q., Yang, Y. H., Yang, L. C.\& Zhou, G. Y. (2016). Above- and belowground biomass allocation in shrub biomes across the northeast tibetan plateau. Plos One, 11, 9.

Olsson, P. A. (1999). Signature fatty acids provide tools for determination of the distribution and interactions of mycorrhizal fungi in soil. FEMS Microbiology Ecology, 29, 303310.

Olsson, P. A., Baath, E.\& Jakobsen, I. (1997). Phosphorus effects on the mycelium and storage structures of an arbuscular mycorrhizal fungus as studied in the soil and roots by analysis of fatty acid signatures. Applied and Environmental Microbiology, 63, 3531-3538.

Opik, M., Metsis, M., Daniell, T. J., Zobel, M.\& Moora, M. (2009). Large-scale parallel 454 sequencing reveals host ecological group specificity of arbuscular mycorrhizal fungi in a boreonemoral forest. New Phytologist, 184, 424-437.

Opik, M., Moora, M., Liira, J.\& Zobel, M. (2006). Composition of root-colonizing arbuscular mycorrhizal fungal communities in different ecosystems around the globe. Journal of Ecology, 94, 778-790.

Opik, M., Moora, M., Zobel, M., Saks, U., Wheatley, R., Wright, F.\& Daniell, T. (2008). High diversity of arbuscular mycorrhizal fungi in a boreal herb-rich coniferous forest. New Phytologist, 179, 867-876.

Opik, M., Vanatoa, A., Vanatoa, E., Moora, M., Davison, J., Kalwij, J. M., . . Zobel, M. (2010). The online database maarjam reveals global and ecosystemic distribution patterns in arbuscular mycorrhizal fungi (glomeromycota). New Phytologist, 188, 223-241.

Pfennigwerth, A. A., Van Nuland, M. E., Bailey, J. K.\& Schweitzer, J. A. (2018). Plant-soil feedbacks mediate shrub expansion in declining forests, but only in the right light. Journal of Ecology, 106, 179-194.

Pianka, E. R. (1970). R-selection and k-selection. American Naturalist, 104, 592-\&. 
Pietikåinen, J., Pettersson, M.\& Bååth, E. (2005). Comparison of temperature effects on soil respiration and bacterial and fungal growth rates. FEMS Microbiology Ecology, 52, 49-58.

Poorter, H. (1993). Interspecific variation in the growth response of plants to an elevated ambient co 2 concentration. In Co2 and biosphere (pp. 77-98): Springer.

Raich, J. W. (1998). Aboveground productivity and soil respiration in three hawaiian rain forests. Forest Ecology and Management, 107, 309-318.

Reyes, B. A., Pendergast, J. S.\& Yamazaki, S. (2008). Mammalian peripheral circadian oscillators are temperature compensated. Journal of Biological Rhythms, 23, 95-98.

Richards, C. L., Bossdorf, O., Muth, N. Z., Gurevitch, J.\& Pigliucci, M. (2006). Jack of all trades, master of some? On the role of phenotypic plasticity in plant invasions. Ecology Letters, 9, 981-993.

Rillig, M. C. (2004). Arbuscular mycorrhizae and terrestrial ecosystem processes. Ecology Letters, 7, 740-754.

Roscher, C., Schumacher, J., Baade, J., Wilcke, W., Gleixner, G., Weisser, W. W., . . Schulze, E. D. (2004). The role of biodiversity for element cycling and trophic interactions: An experimental approach in a grassland community. Basic and Applied Ecology, 5, 107121.

Ross, M. A.\& Harper, J. L. (1972). Occupation of biological space during seedling establishment. Journal of Ecology, 60, 77-\&.

Schmidtke, A., Rottstock, T., Gaedke, U.\& Fischer, M. (2010). Plant community diversity and composition affect individual plant performance. Oecologia, 164, 665-677.

Schüßler, A., Schwarzott, D.\& Walker, C. (2001). A new fungal phylum, the glomeromycota: Phylogeny and evolution* *dedicated to manfred kluge (technische universität darmstadt) on the occasion of his retirement. Mycological Research, 105, 14131421.

Semchenko, M., Leff, J. W., Lozano, Y. M., Saar, S., Davison, J., Wilkinson, A., . . Bardgett, R. D. (2018). Fungal diversity regulates plant-soil feedbacks in temperate grassland. Science Advances, 4, eaau4578.

Sepp, S.-K., Davison, J., Jairus, T., Vasar, M., Moora, M., Zobel, M.\& Opik, M. (2019). Nonrandom association patterns in a plant-mycorrhizal fungal network reveal hostsymbiont specificity. Molecular Ecology, 28, 365-378.

Sharma, M. P.\& Buyer, J. S. (2015). Comparison of biochemical and microscopic methods for quantification of arbuscular mycorrhizal fungi in soil and roots. Applied Soil Ecology, 95, 86-89.

Smith, S. E.\& Read, D. J. (2008). Mycorrhizal symbiosis, 3rd edn. Cambridge: UK: Academic Press Ltd.

Soka, G. E.\& Ritchie, M. E. (2016). Contributions of am fungi and soil organic matter to plant productivity in tropical savanna soils under different land uses. Rhizosphere, 1, 4552.

Tilman, D. (1994). Competition and biodiversity in spatially structured habitats. Ecology, 75, 2-16.

Tilman, D. (1995). Biodiversity: Population versus ecosystem stability. Ecology, 77, 350-363.

Tilman, D. (1997). The influence of functional diversity and composition on ecosystem processes. Science, 277, 1300-1302.

Tilman, D. (2001). Functional diversity. In S. A. Levin (Ed.), Encyclopedia of biodiversity (pp. 109-120). New York: Elsevier.

Tilman, D., Isbell, F.\& Cowles, J. M. (2014). Biodiversity and ecosystem functioning. In D. J. Futuyma (Ed.), Annual review of ecology, evolution, and systematics, vol 45 (Vol. 45, pp. 471-493). Palo Alto: Annual Reviews. 
Tilman, D., Reich, P. B., Knops, J., Wedin, D., Mielke, T.\& Lehman, C. (2001). Diversity and productivity in a long-term grassland experiment. Science, 294, 843-845.

Tylianakis, J., Martínez-García, L., Richardson, S., Peltzer, D.\& Dickie, I. (2018). Symmetric assembly and disassembly processes in an ecological network (Vol. 21).

Tylianakis, J. M., Didham, R. K., Bascompte, J.\& Wardle, D. A. (2008). Global change and species interactions in terrestrial ecosystems. Ecology Letters, 11, 1351-1363.

Urcelay, C.\& Díaz, S. (2003). The mycorrhizal dependence of subordinates determines the effect of arbuscular mycorrhizal fungi on plant diversity. Ecology Letters, 6, 388-391.

van der Heijden, M. G. A., Boller, T., Wiemken, A.\& Sanders, I. R. (1998). Different arbuscular mycorrhizal fungal species are potential determinants of plant community structure. Ecology, 79, 2082-2091.

van der Wal, A., van Veen, J. A., Smant, W., Boschker, H. T. S., Bloem, J., Kardol, P., . . de de Boer, W. (2006). Fungal biomass development in a chronosequence of land abandonment. Soil Biology \& Biochemistry, 38, 51-60.

Vaughn, K. J.\& Young, T. P. (2015). Short-term priority over exotic annuals increases the initial density and longer-term cover of native perennial grasses. Ecological Applications, 25, 791-799.

Vestberg, M., Palojärvi, A., Pitkänen, T., Kaipainen, S., Puolakka, E.\& Keskitalo, M. (2012). Neutral lipid fatty acid analysis is a sensitive marker for quantitative estimation of arbuscular mycorrhizal fungi in agricultural soil with crops of different mycotrophy. Agricultural and Food Science, 21, 12-27.

Waide, R. B., Willig, M. R., Steiner, C. F., Mittelbach, G., Gough, L., Dodson, S. I., . . . Parmenter, R. (1999). The relationship between productivity and species richness. Annual Review of Ecology and Systematics, 30, 257-300.

Wang, G., Schultz, P., Tipton, A., Zhang, J., Zhang, F.\& Bever, J. D. (2019). Soil microbiome mediates positive plant diversity-productivity relationships in late successional grassland species. Ecology Letters, 22, 1221-1232.

Way, D. A.\& Yamori, W. (2014). Thermal acclimation of photosynthesis: On the importance of adjusting our definitions and accounting for thermal acclimation of respiration. Photosynthesis Research, 119, 89-100.

Weidlich, E. W. A., von Gillhaussen, P., Max, J. F. J., Delory, B. M., Jablonowski, N. D., Rascher, U.\& Temperton, V. M. (2018). Priority effects caused by plant order of arrival affect below-ground productivity. Journal of Ecology, 106, 774-780.

Willers, C., van Rensburg, P. J. J.\& Claassens, S. (2015). Phospholipid fatty acid profiling of microbial communities-a review of interpretations and recent applications. Journal of Applied Microbiology, 119, 1207-1218.

Wolf, D. C., Dao, T. H., Scott, H. D.\& Lavy, T. L. (1989). Influence of sterilization methods on selected soil microbiological, physical, and chemical properties. Journal of Environmental Quality, 18, 39-44.

Yachi, S.\& Loreau, M. (1999). Biodiversity and ecosystem productivity in a fluctuating environment: The insurance hypothesis. Proceedings of the National Academy of Sciences of the United States of America, 96, 1463-1468. 